\title{
Comparação de Estimadores do Parâmetro de Longa Memória do Modelo ARFIMA(p,d,q)
}

Cristina Baptista Moura

DISSERTAÇAO APRESENTADA

$\mathrm{AO}$

INSTITUTO DE MATEMÁTICA E ESTATÍSTICA

DA

UNIVERSIDADE DE SÃO PALLO

PARA OBTENGÃO DO GRAL DE MESTRE

EMI

ESTATÍSTICA

Área de Concentração: Séries Temporais Orientador : Profa. Dra. Clélia M. C. Toloi 


\title{
Comparação de Estimadores do Parâmetro de Longa Memória do Modelo ARFIMA(p,d,q)
}

\author{
Cristina Baptista Moura
}

\author{
Este exemplar corresponde à redação \\ final da dissertação devidamente corrigida \\ e apresentada por Cristina Baptista Moura e aprovada \\ pela Comissão Julgadora.
}

São Paulo, 15 de Janeiro de 1998

Banca Examinadora :

- Profa. Dra. Clélia Maria de Castro Toloi (orientadora) - IME-USP

- Prof. Dr. Pedro Alberto Morettin - IME-USP

- Prof. Dr. Denisard Cnéio de Oliveira Alves - FEA-USP 
à minha família e principalmente ao meu avô que mesmo não estando mais entre nós sempre esteve ao meu lado 


\section{Agradecimentos}

Agradeço a todos os professores deste Instituto por tudo que pude aprender através deles. Agradeço à Clélia por seu profissionalismo e seu lado humano. Aos amigos novos que ganhei aqui em Sào Paulo. Eliana por sua ternura, Erika pela infinita tranquilidade, Patrícia e Flávio pelo exemplo de companheirismo, Péricles pelo sua eterna alegria, Aldy pelo jeito mineiro, ou seja, acolhedor, Audrey, Francisco e Rafael pelo ambiente familiar que trouxeram a nós. Um agradecimento especial devo à Liane com quem divido tantos espaços e histórias. A Renata, amiga do Rio que se aventurou comigo a fazer o mestrado aqui, agradeço por sua amizade. Nào posso esquecer dos amigos maravilhosos do Rio, principalmente Simone e Henry, que mesmo de longe me ajudaram muito. Uma agradecimento super especial ao Francisco (Fran), por sua eterna paciência e apoio, fazendo com que tudo fique fácil. Pelo exemplo e amizade em todos os momentos agradeço por ter uma Màe tão especial. Entretanto, devo agradecer em primeiro lugar à Deus por ter me envolvido de pessoas tão especiais. 


\section{Resumo}

Este trabalho tem como objetivo comparar seis métodos de estimação do

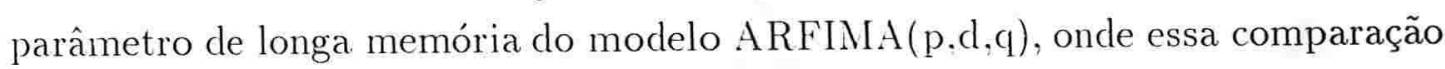
é feita através de simulações e do ajuste desse modelo a uma série hidrológica. Os métodos utilizados foram: método da autocorrelação de ordem 1(Gupta \& Kumar (1991)), método da autocorrelaçào generalizado (Gupta Ji (1992)), método do periodograma (Geweke \& Porter-Hudak (1983)). método do periodograma suavizado (Reisen (1994)), método de máxima verossimilhança (Fox \& Taqqu (1986)) e o método das ondaletas (Jensen (1996)). Foram simuladas 1000 amostras de três tamanhos diferentes, 128,256 e 512, para os modelos $\operatorname{ARFIMA}(0, \mathrm{~d}, 0)$, $\operatorname{ARFIMA}(1, \mathrm{~d}, 0)$ e $\operatorname{ARFIMA}(0, \mathrm{~d}, 1)$. Quanto à série hidrológia, comparamos os estimadores através do ajuste de modelos ARFIMA(p,d.q) à série, utilizando os seis métodos de estimação. Também foi feita a previsào das doze últimas observações da série para comparar os estimadores através do poder de previsão dos mesmos. Além disso, ajustamos outros modelos mais tradicionais à essa série para compará-los ao modelo de longa memória. 


\section{Abstract}

The objective of this work is to compare six estimation methods of the long memory parameter of the ARFIMA(p.d.q) model. This comparison is clone by sample simulations of this model and by the adjustment of this model to a hydrological series. The methods used in this comparison were: method of auto-correlation at lag one (Gupta (E Lumar (1991)). method of generalized auto-correlation (Gupta (t Ji (1992)). method of the periodogram (Gewekw (E Porter-Hudak (1983)), method of the smoothed periodogram (Reisen (1994)). method of the maximum likelihood (Fox ( Taqqu (1986)) and the method of the wavelet (Jensen (1996)). We simulated 1000 samples of three different sizes (128. 256 and 512) to three ARFIMA(p,d,q) models: ARFIMA(0,d,0), ARFIMA(1,d,0) and $\operatorname{ARFIMA}(0, d, 1)$. The hydrological series was used in the adjustment of the ARFIMA(p,d,q) models. to the six estimation methods referre mentioned before, with the objective to compare these estimators. We also made the forecasting to the last twelve observations of the series to compare the estimators by the forecasting power of them. The adjustment of traditional models like $\operatorname{ARIMA}(p, d, q)$ and $\operatorname{AR}(p)$ was made to compare them to the long memory model. 


\section{Conteúdo}

Capítulo 1 Introdução 1

Capítulo 2 Definições e Resultados 4

$\begin{array}{lll}\text { Capítulo } 3 & \text { Modelos Lineares } & 14\end{array}$

3.1 Modelos ARMA(p,q) . . . . . . . . . . . . . . 16

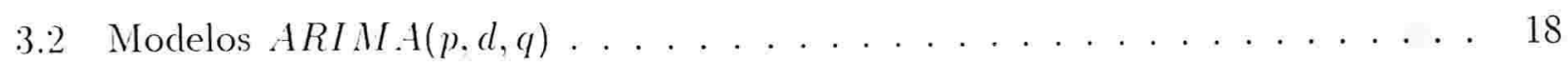

Capítulo 4 Modelos de Longa Memória $\quad 22$

4.1 Introdução . . . . . . . . . . . . . . . . . . . . 22

4.2 Modelo ARIMA Fracionário (ARFIMA) . . . . . . . . . . 25

Capítulo 5 Estimação dos Parâmetros em Modelos ARFIMA(p,d,q) 33

5.1 Métodos de Estimação de $d \ldots \ldots$. . . . . . . . . . . . . . . 33

5.1 .1 Introduçào . . . . . . . . . . . . . . . . . . . 33

5.1 .2 Método da Função de Autocorrelação de Ordem 1 . . . . . . . . . 34

5.1 .3 Método da Função de Autocorrelação Generalizado . . . . . . . . . 35

5.1 .4 Método da Função de Autocorrelaçào Parcial . . . . . . . . . . . . 37

5.1.5 Método da Regressào Utilizando o Periodograma . . . . . . . . . . 37 
5.1.6 Método de Regressào L'tilisando o Periodograma Suavizado . . . . . . 42

$5.1 . i$ Método de Máxima Verossimilhança . . . . . . . . . . . . . . 44

5.1 .8 Método de Ondaletas . . . . . . . . . . . . . . . 47

5.2 Algorítmo para ajustamento de um modelo $\operatorname{ARFIMA}(\mathrm{p}, \mathrm{d}, \mathrm{q}) \ldots \ldots$

Capítulo 6 Simulações $\quad 53$

6.1 Resultados para os modelos ARFIMA $(0, \mathrm{~d}, 0) \ldots \ldots \ldots \ldots$

6.2 Resultados para os modelos ARFiMA(1,d,0) . . . . . . . . . . 66

6.3 Resultados para o modelo ARFIMA(0.d.1) . . . . . . . . . . 77

$\begin{array}{lll}\text { Capítulo } 7 & \text { Aplicação a uma série real } & 87\end{array}$

T.1 Série de Vazão do Rio São Marcos . . . . . . . . . . . . . . . . 87

7.1.1 Ajustamento dos modelos . . . . . . . . . . . . . 9 90

7.1 .2 Comparaçào dos modelos . . . . . . . . . . . . . 103

$\begin{array}{lll}\text { Capítulo } 8 & \text { Conclusão } & 108\end{array}$

$\begin{array}{lr}\text { Bibliografia } & 111\end{array}$

Apêndice A Programas para estimação dos parâmetros $\quad 114$

Apêndice B Programa para previsão do modelo SARF $I M A(6, d, 0) \times(0,1,1)_{12} 127$

$\begin{array}{lll}\text { Apêndice C } & \text { Logarítmo da série hidrológica } & 128\end{array}$ 


\section{Capítulo 1}

\section{Introdução}

No estudo de Séries Temporais encontramos vários modelos que se aplicam aos mais diferentes casos e um dos modelos mais utilizados é o $A R I M A(p, d, q)$, onde $d$ é o parâmetro de diferença inteira da série. que tem como objetivo transformar uma série não estacionária em estacionária. Além disso, esse modelo supõe que as observaçòes distanciadas por um longo intervalo no tempo são independentes, ou seja, supõem independência assintótica entre as observaçòes. Entretanto, na prática, encontramos série temporais que nào apresentam essa independência e a esse tipo de série denominamos de série de longa memória. Para modelar esse tipo de série desenvolveu-se o modelo $A R I M A(p, d, q)$ fracionário. ou $A R F I M A(p, d, q)$. que é uma generalizaçào do modelo $A R I M A(p, d, q)$, pois no novo modelo o parâmetro d assume valores fracionários.

O primeiro modelo desenvolvido para ajustar séries de longa memória foi apresentado por Mandelbrot \&: Ness (1968) e chamado de modelo de ruído Gaussiano fracionário. Granger \& Joyeux (1980) e Hosking (1981) demonstram que o processo $A R I M A(0, d, 0)$ fracionário apresenta o mesmo comportamento de longa memória do processo de ruído Gaussiano fracionário. Geweke \& Porter Hudak (1983) mostram a equivalência desses dois modelos. A partir da teoria desenvolvida para o modelo $A R F I M A(0, d, 0)$ sua generalização para o mo-

delo ARFIM A $(p, d, q)$ é imediata. Granger \& Joyeux (1980) e Hosking (1981) demonstram todas as propriedades desses modelos, que mais tarde são utilizadas no desenvolvimento de estimadores do parâmetro $d$.

O primeiro estimador de $d$, desenvolvido por Geweke \& Porter-Hudak (1983), foi baseado 
na funçào de densidade espectral de um processo ARFIMIA(p.d.cp) e utiliza o periodograma simples como estimador desta funçào. Reisen ( 1994) demonstra que utilizar o periodograma suavizado como estimador da função de densidade espectral produz um estimador mais eficiente do parâmetro $d$. surgindo assim um novo estimador. O mais simples dos estimadores foi desenvolvido por Gupta e Kumar (1991) e é baseado na função de autocorrelação de um processo ARFIMA $A(0$, d.0). Este estimador utiliza apenas a autocorrelação amostral de ordem 1 para estimar $d$. Gupta \& Ji (1992) apresentaram uma generalização deste estimador, onde são utilizadas várias autocorrelações amostrais da série. Vinod (1991) desenvolveu um estimador que se baseia na função de autocorrelaçào parcial do processo $\operatorname{ARFIMA}(\mathrm{p}, \mathrm{d}, \mathrm{q})$ e utiliza autocorrelaçòes parciais amostrais da série para estimar $d$. O estimador de máxima verossimilhança foi desenvolvido por Fox \& Tacqu (1986). O mais recente dos estimadores se baseia na teoria dos coeficientes de "wavelets" e foi desenvolvido por Jensen (1996).

Este trabalho tem como objetivo comparar esses estimadores através de simulações e de ajustes de uma série hidrológica com claro comportamento de longa memória. Nas simulaçòes procuramos apresentar vária situaçòes diferentes para que se pudesse comparar os estimadores em relação ao tamanho da amostra. aos valores do parâmetro $d$ e a algumas possibilidades de modelos $A R F I M A(p, d . q)$. Ao utilizarmos a série real podemos testar o ajuste e o poder de previsào desses modelos e compará-los com modelos mais tradicionais como o $\operatorname{AR}(p)$ e $\operatorname{ARIMA}(p \cdot d, q)$.

No Capítulo 2 apresentamos alguns resultados e definiçòes que serão utilizados e citados nos capítulos posteriores.

O Capítulo 3 contém um resumo das propriedades e da teoria dos modelos $\operatorname{ARIMA}(\mathrm{p}, \mathrm{d}, \mathrm{q})$, com o objetivo de apresentar as informaçòes necessárias para o entendimento do Capítulo 4 que apresenta a teoria dos modelos $\operatorname{ARFIMA}(\mathrm{p}, \mathrm{d}, \mathrm{q})$ e suas propriedades.

O Capítulo j descreve todos os métodos de estimaçào do parâmetro $d$ do modelo ARF IMA $(p, d, q)$, explicando suas origens e apresentando suas propriedades.

Os resultados das simulações são apresentados no Capítulo 6, onde são feitas as comparações dos estimadores para três modelos: $A R F I . M A(0, d, 0), A R F I M A(1, d, 0)$ e $A R F I M A(0, d, 1)$.

A aplicação desses modelos à série hidrológica é apresentada no C'apítulo 7, onde comparamos os modelos ARFIMA(p,d,q) ajustados com os modelos AR(p) e ARIMA(p,d,q), 
CAPÍtLlo 1. INTRODLÇÃO

também ajustados à série. 


\section{Capítulo 2}

\section{Definições e Resultados}

Definição 2.1 Um processo estocústico $\left\{X_{t}\right\}=\left\{X^{\prime}(t, \omega) . t \in T, \omega \in \Omega\right\}$ é uma família de variáveis aleatórias definidas num espaço de probabilidade $(\omega, \Lambda, P)$ e indexadas pelos elementos de um conjunto de parâmetros $T$.

Na realidade, um processo estocástico é uma função de dois argumentos, $X(t, \omega), t \in T$, $\omega \in \Omega$. Para cada $t \in T$, a função $X^{\prime}(t, \cdot)$ é mensurável relativamente a $\Omega$. Por outro lado, para cada $\omega \in \Omega$, fixado, obteremos uma funçào $X^{\prime}(\cdot, \omega)$ de $t: T \mapsto \mathbb{R}$, que é chamada trajetória, realização, função amostral do processo ou série temporal.

Definição 2.2 Dado um processo estocástico $\left\{X_{t}\right\}$, definimos suas distribuições finitodimensionais por

$$
F\left(x_{1}, \ldots, x_{n} ; t_{1}, \ldots, t_{n}\right)=\operatorname{Prob}\left(X_{t_{1}} \leq x_{1}, \ldots, X_{t_{n}} \leq x_{n}\right),
$$

para todo $n \geq 1$ e $t_{1}, \ldots, t_{n} \in T$.

Definição 2.3 As funções média e autocovariância de um processo $\left\{X_{t}\right\}$ são dadas por

$$
\begin{aligned}
& E\left(X_{t}\right)=\int_{-\infty}^{\infty} x d F(x ; t)=\mu_{x}(t) \\
\operatorname{Cov}\left[X_{t_{1}}, X_{t_{2}}\right]= & \int_{-\infty}^{\infty} \int_{-\infty}^{\infty}\left[x_{1}-\mu_{x}\left(t_{1}\right)\right]\left[x_{2}-\mu_{x}\left(t_{2}\right)\right] d F\left[x_{1}, x_{2} ; t_{1}, t_{2}\right] \\
= & E\left[X_{t_{1}}, X_{t_{2}}\right]-E\left[X_{t_{1}}\right] E\left[X_{t_{2}}\right] \\
= & \gamma_{x}\left(t_{1}, t_{2}\right) .
\end{aligned}
$$


Em particular. se $t_{1}=t_{2}=t$. temos a variância do processo

$$
\operatorname{Var}\left(X_{t}\right)=\int_{-\infty}^{\infty}\left[x-\mu_{x}(t)\right]^{2} d F(x ; t)=E\left[X_{t}^{2}\right]-E^{2}\left[X_{t}\right]=\gamma_{x}(t, t)
$$

Definição 2.4 Um processo estocástico $\left\{X_{t}\right\}$ é estritamente estacionário se todas as distribuições finito-dimensionais (2.1) permanecem as mesmas sob uma translação do tempo. ou seja,

$$
F\left(x_{1}, \ldots x_{n} ; t_{1}+\tau \ldots, t_{n}+\tau\right)=F\left(x_{1}, \ldots, x_{n} ; t_{1}, \ldots, t_{n}\right),
$$

para quaisquer $t_{1}, \ldots, t_{n} ; \tau \in T$.

Definição 2.5 Um processo estocástico $\left\{X_{t}\right\}$ é tstacionário de segunda ordem, ou fracamente estacionário se

(a) $E\left[X_{t}\right]=\mu_{x}$, constante. $\forall t \in T$.

(b) $E\left[X_{t}^{2}\right]<\infty, \forall t \in T$.

(c) $\gamma_{x}\left(t_{1}, t_{2}\right)=\gamma_{x}\left(t_{1}-t_{2}\right)$ só depende da defasagem $\left|t_{1}-t_{2}\right|, \forall t_{1}, t_{2} \in T$.

Nota: No decorrer do texto nos referiremos a essa defasagem por ordem.Por exemplo, seja $k=\left|t_{1}-t_{2}\right|$, entào $\gamma_{x}(k)$ será a autocorrelaçào de ordem $k$ da série $X_{t}$.

Definição 2.6 (a) Seja $\left(X_{1}, \ldots X_{r}\right)$ uma variável aleatória r-dimensional com $E\left|X_{j}\right|^{r}<\infty, j=1, \ldots, r$ onde $X_{j}$ são reais ou complexas. O cumulante conjunto de ordem $r$ é dado por

$$
\operatorname{Cum}\left(X_{1}, \ldots, X_{r}\right)=\sum_{\nu}(-1)^{p-1} !\left(E \prod_{j \in \nu_{1}} X_{j}\right) \ldots\left(E \prod_{j \in \nu_{p}} X_{j}\right)
$$

onde a soma é sobre todas as partiçòes $\nu=\left(\nu_{1}, \ldots, \nu_{p}\right)$ dos inteiros $(1, \ldots, r)$.

(b) Seja uma série temporal $\left\{X_{t}, t=0, \pm 1, \ldots\right\}$ satisfazendo $E\left|X_{t}\right|^{k}<\infty$, então

$$
C_{x}\left(t_{1} \ldots, t_{r}\right)=\operatorname{Cum}\left(X_{t_{1}}, \ldots X_{t_{r}}\right)
$$

é denominada função cumulante conjunta de ordem $r$ da série $X_{t}$. 
Definição 2.7 Suponha um processo estacionário $\left\{X_{t}\right\} . t=0 . \pm 1 \ldots$ com função de autocovariância (f.a.c) $\hat{j}_{x}(k) . k=0 . \pm 1 \ldots$ satisfazendo

$$
\sum_{u=-\infty}^{\infty}\left|\gamma_{x}(u)\right|<\infty
$$

então o espectro de $\left\{X_{t}\right\}$. na frequência $\lambda$, é dado por

$$
f_{x}(\lambda)=(2 \pi)^{-1} \sum_{k=-\pi}^{\pi} \exp (-i \lambda k) \gamma_{x}(k)
$$

para $-\infty<\lambda<\infty$.

Demonstra-se que o espectro $f_{x}(\lambda)$ definido por (2.8) é limitado. nào negativo e uniformemente contínuo. Além disso. $f_{x}(\lambda)$ é par e periódico de período $2 \pi$.

Como $f_{x}(\lambda)$ é par e periódico de período $2 \pi$. basta tomar $\lambda \in[-\pi . \pi]$ e utilizar o intervalo $[0, \pi]$ como domínio fundamental da definição do espectro $f_{x}(\lambda)$.

A relaçào $(2.8)$ pode ser invertida e a funçào de autocovariância $\gamma_{x}(k)$ pode ser escrita como

$$
\gamma_{x}(k)=\int_{-\pi}^{\pi} \exp (i \lambda k) f_{x}(\lambda) d \lambda
$$

para $k=0, \pm 1 \ldots$. No caso particular de $k=0$, temos

$$
\operatorname{Var}\left(X_{t}\right)=\int_{-\pi}^{\pi} f_{x}(\lambda) d \lambda
$$

Definição 2.8 Sejam $\left\{X_{t}\right\}$ e $\left\{Y_{t}\right\}$ dois processos conjuntamente estacionários com $E\left[X_{t}\right]=$ $\mu_{x}$ e $E\left[Y_{t}\right]=\mu_{y}$.

(a) A função de correlação cruzada de ordem $m$ entre $X$ e $Y$ é dada por

$$
\gamma_{x y}(m)=E\left[\left(X_{t-m}-\mu_{x}\right)\left(Y_{t}-\mu_{y}\right)\right], \quad m=0 . \pm 1, \ldots .
$$

(b) A funçào de correlaçào cruzada de ordem $m$ entre $\mathrm{X}$ e $Y$ é dada por

$$
\rho_{x y}(m)=\frac{\gamma_{x y}(m)}{\sqrt{\gamma_{x}(0) \gamma_{y}(0)}} . \quad m=0 . \pm 1 \ldots .
$$


Definição 2.9 Seja $X_{n}, n=0 . \pm 1 \ldots$ um processo estacionário real. A transformada finita de Fourier dos valores $X_{0}, X_{1} \ldots X_{T-1}$ de $X_{n}$ é dada por

$$
d_{x}^{T}(\lambda)=\sum_{t=0}^{T-1} X_{t} \exp (-i \lambda t) . \quad-\infty<\lambda<\infty .
$$

A transformada discreta de Fourier dos valores $X_{0}, \ldots, X_{T-1}$ de $X_{n}$ é dada por

$$
d_{x}^{T}\left(\frac{2 \pi s}{T}\right), s=0,1, \ldots, T-1
$$

\section{Propriedades:}

1. $d_{x}^{T}(\lambda+2 \pi)=d_{x}^{T}(\lambda)$.

2. $\overline{d_{x}^{T}(\lambda)}=d_{x}^{T}(-\lambda)$, se $X_{t}$ é real.

3. $d_{\alpha x+\beta y}^{T}(\lambda)=\alpha d_{x}^{T}(\lambda)+\beta d_{x}^{T}(\lambda)$, se $\alpha, \beta$ são escalares e $X_{t}, Y_{t}$ são processos estacionários.

A relação $(2.11)$ pode ser invertida e a série $X_{t}$ pode ser escrita como

$$
X_{t}=(2 \pi)^{-1} \int_{0}^{2 \pi} \exp (i \lambda t) d_{x}^{T}(\lambda) d \lambda, \quad t=0,1, \ldots, T-1
$$

ou

$$
X_{t} \cong T^{-1} \sum_{s=0}^{T-1} \exp \left(\frac{i 2 \pi s t}{T}\right) d_{x}^{T}\left(\frac{2 \pi s}{T}\right), \quad t=0,1, \ldots, T-1
$$

Notação: Se $x$ e $y$ são números reais, escreveremos $x \equiv y(\bmod \alpha)$ quando a diferença $x-y$ for um múltiplo de $\alpha$.

Definição 2.10 Seja $X_{t}, t=0, \pm 1, \ldots$ uma série temporal estacionária, o periodograma dos valores $X_{0}, X_{1}, \ldots, X_{T-1}$ de $X_{t}$ é dado por

$$
I_{x}^{T}(\lambda)=(2 \pi T)^{-1}\left|d_{x}^{T}(\lambda)\right|^{2}, \lambda \not \equiv 0(\bmod 2 \pi) .
$$


Teorema 2.1 Se $X_{t}, t=0 . \pm 1, \ldots$ é uma série temporal satisfazendo (2.ĩ), então

$$
\begin{aligned}
E\left(I_{x}^{T}(\lambda)\right) & =\frac{1}{2 \pi T} \int_{-\pi}^{\pi}\left[\frac{\operatorname{sen}\left(\frac{T(\lambda-\alpha)}{2}\right)}{\operatorname{sen}\left(\frac{\lambda-\alpha}{2}\right)}\right]^{2} f_{x}(\alpha) d x \\
& +\frac{1}{2 \pi T}\left[\frac{\operatorname{sen}\left(\frac{T, \lambda}{2}\right)}{\operatorname{sen}\left(\frac{\lambda}{2}\right)}\right]^{2} \mu_{x}^{2},
\end{aligned}
$$

para $-\infty<\lambda<\infty$. No caso $\lambda \not \equiv 0(\bmod 2 \pi)$, o último termo de $(2.14)$ é pequeno e notamos que $E\left(I_{x}^{T}(\lambda)\right)$ é aproximadamente igual a uma média ponderada do espectro, com peso concentrado na vizinhança de $\lambda$. O termo $\left[\frac{\operatorname{sen}(T, / 2)}{\operatorname{sen}(\lambda / 2)}\right]^{2}$ é denominado núcleo de Fejér.

Corolário 2.1 Sob as mesmas condiçòes do Teorema 2.1 . se $\lambda \not \equiv 0(\bmod 2 \pi)$, então $I_{x}^{T}(\lambda)$ é um estimador assintoticamente nào viciado de $f_{x}(\lambda)$.

Teorema 2.2 Sob as condições do Teorema 2.1 e se

$$
\sum_{k}|k|\left|\gamma_{i x}(k)\right|<\infty
$$

então

$$
E\left(I_{x}^{T}(\lambda)\right)=f_{x}(\lambda)+\frac{1}{2 \pi T}\left[\frac{\operatorname{sen}\left(\frac{T \lambda}{2}\right)}{\operatorname{sen}\left(\frac{\lambda}{2}\right)}\right]^{2} \mu_{x}^{2}+O\left(T^{-1}\right) .
$$

O termo $O\left(T^{-1}\right)$ é uniforme em $\lambda$.

Teorema 2.3 Se $X_{t}$ é Gaussiano e $I_{x}^{T}(\lambda)$ é dado por $(2.14)$ para $\lambda, \mu \neq \equiv 0(\bmod 2 \pi)$, temos que

$$
\begin{aligned}
\operatorname{Cov}\left(I_{x}^{T}(\lambda), I_{x}^{T}(\mu)\right) & =\left(\left[\frac{\operatorname{sen}\left(\frac{T(\lambda+\mu)}{2}\right)}{T \operatorname{sen}\left(\frac{\lambda+\mu}{2}\right)}\right]^{2}+\left[\frac{\operatorname{sen}\left(\frac{T(\lambda-\mu)}{2}\right)}{T \operatorname{sen}\left(\frac{\lambda-\mu}{2}\right)}\right]^{2}\right) f_{x}(\lambda) \\
& +O\left(T^{-1}\right) .
\end{aligned}
$$


Teorema 2.4 Nas mesmas condiçòes do Teorema 2.3. se $\mu=2 \pi r / T . \lambda=2 \pi s / T$, com $r, s, r \pm s \not \equiv 0(\bmod T)$, inteiros. entào

$$
\begin{aligned}
& \operatorname{Var} I_{x}^{T}(\lambda)=f_{x}^{2}(\lambda)+O\left(T^{-1}\right) . \\
& \operatorname{Cov}\left(I_{x}^{T}(\lambda), I_{x}^{T}(\mu)\right)=O\left(T^{-1}\right) .
\end{aligned}
$$

Demonstrações: ver Brillinger(1981).

Teorema 2.5 Se $X_{t}$ é estritamente estacionário satisfazendo

$$
\sum_{u_{1}, \ldots, u_{k-1}}^{\infty} \mid\left(C_{x}\left(u_{1} \ldots, u_{k-1}\right) \mid<\infty\right.
$$

e $s_{j}^{T}$ um inteiro com

$$
\lambda_{j}^{T}=\frac{2 \pi s_{j}^{T}}{T} \rightarrow \lambda_{j}
$$

quando $T \rightarrow \infty, j=1,2, \ldots, J$, entào

(a) para $2 \lambda_{j}^{T}, \lambda_{j}^{T} \pm \lambda_{k}^{T} \not \equiv 0(\bmod 2 \pi), 1 \leq j<m \leq J$ e $T=1,2, \ldots$ temos que

$$
I_{x}^{T}\left(\lambda_{j}^{T}\right) \stackrel{\mathcal{D}}{\rightarrow} f_{x}\left(\lambda_{j}\right) \frac{Y_{2}^{2}}{2}
$$

independentes;

(b) para $\lambda= \pm \pi, \pm 3 \pi, \ldots$, temos

$$
I_{x}^{T}(\lambda) \stackrel{\mathcal{D}}{\rightarrow} f_{x}(\lambda) \lambda_{1}^{2}
$$

independentes e

(c) para $\lambda \equiv 0(\bmod 2 \pi)$ temos

$$
I_{x}^{T}(\lambda) \stackrel{\mathcal{D}}{\rightarrow} \begin{cases}f_{x}(\lambda) \backslash \frac{2}{1} & \text { se } \mu_{x}=0 \\ f_{x}(\lambda) \backslash{ }_{1}^{2}(\psi) & \text { se } \mu_{x} \neq \equiv 0\end{cases}
$$

onde $\psi=\left|\mu_{x}\right| \sqrt{T /\left(2 \pi f_{x}(\lambda)\right)}$ é o parâmetro de não centralidade do qui-quadrado. 
Podemos notar que. embora o periodograma seja assintoticamente nào viciado, ele não é consistente (Brillinger. 1981).

Pelo Teorema 2.5. se $s^{T}$. inteiro $\operatorname{com} \frac{2 \pi s^{T}}{T} \rightarrow \lambda$ e $2\left[s^{T}+j\right] \not \equiv 0(\bmod T)$, então $I_{x}^{T}\left(2 \pi\left[s^{T}+j\right] / T\right) . j=0 . \pm 1 \ldots \pm \pm m$, sào aproximadamente independentes com distribuição assintótica $f_{x}(\lambda) \gamma_{2}^{2} / 2$.

Definição $2.11 \mathrm{Um}$ novo estimador para $f_{x}(\lambda)$, denominado periodograma suavizado, é dado por

$$
f_{x}^{T}(\lambda)= \begin{cases}(2 m+1)^{-1} \sum_{j=-m}^{m} I_{x}^{T}\left(\frac{2 \pi\left[s^{T}+j\right]}{T}\right) & \text { se } \lambda \neq 0(\bmod \pi) \\ (2 m)^{-1}\left[\sum_{j=-m}^{-1} I_{x}^{T}\left(\lambda+\frac{2 \pi j}{T}\right)+\sum_{j=1}^{m} I_{x}^{T}\left(\lambda+\frac{2 \pi j}{T}\right)\right] & \text { se } \lambda \equiv 0(\bmod \pi) \\ & \text { ou } \lambda= \pm \pi, \pm 3 \pi, \ldots \\ m^{-1} \sum_{j=1}^{m} I_{x}^{T}\left(\lambda-\frac{\pi}{T}+\frac{2 \pi j}{T}\right) & \text { e } T \text { é par } \\ & \text { se } \lambda= \pm \pi, \pm 3 \pi, \ldots \\ & \text { e } T \text { é impar }\end{cases}
$$

que é uma média das ordenadas do periodograma em torno de $\lambda$.

Definindo

$$
F_{T}(\lambda)=(2 \pi T)^{-1}\left[\frac{\operatorname{sen}(T \lambda / 2)}{\operatorname{sen}(\lambda / 2)}\right]^{2}
$$

que é o núcleo de Fejér.

$$
\begin{aligned}
& A_{T}^{m}(\lambda)=(2 m+1)^{-1} \sum_{j=-m}^{m} F_{T}\left(\lambda-\frac{2 \pi j}{T}\right) \\
& B_{T}^{m}(\lambda)=(2 m)^{-1} \sum_{j=-m}^{-1} F_{T}\left(\lambda-\frac{2 \pi j}{T}\right)+\sum_{j=1}^{m} F_{T}\left(\lambda-\frac{2 \pi j}{T}\right) \mathrm{e} \\
& C_{T}^{m}(\lambda)=(2 m)^{-1} \sum_{j=1}^{m}\left(F_{T}\left(\lambda-\frac{\pi}{T}+\frac{2 \pi j}{T}\right)\right)+F_{T}\left(\lambda+\frac{\pi}{T}-\frac{2 \pi j}{T}\right)
\end{aligned}
$$


para $-\infty<\lambda<\infty$. Podemos notar que $A_{T}^{m}$. $B_{T}^{m}$ e $C_{T}^{m}$ sào nào negativos, têm integral um sobre o intervalo $[-\pi . \pi]$ com periodicidade $2 \pi$.

Teorema 2.6 Se $X_{t}, t=0, \pm 1 \ldots$ e $\sum_{k=-\infty}^{\infty}\left|\gamma_{i x}(k)\right|<\infty$. então

$$
E\left[f_{x}^{T}(\lambda)\right]= \begin{cases}\int_{-\pi}^{\pi} A_{T}^{m}(\alpha) f_{x}\left(\frac{2 \pi s^{T}}{T}-\alpha\right) d \alpha & \text { se } \lambda \not \equiv 0(\bmod 2 \pi) \\ \int_{-\pi}^{\pi} B_{T}^{m}(\alpha) f_{x}(\lambda-\alpha) d \alpha & \text { se } \lambda \equiv 0(\bmod 2 \pi) \\ & \text { eu } \lambda= \pm \pi, \pm 3 \pi, \ldots \\ \int_{-\pi}^{\pi} C_{T}^{m}(\alpha) f_{x}(\lambda-\alpha) d \alpha & \text { se } \lambda= \pm \pi, \pm 3 \pi, \ldots \\ & \text { e } T \text { é impar }\end{cases}
$$

Corolário 2.2 Se $\lambda-\frac{2 \pi s^{T}}{T} \rightarrow O\left(T^{-1}\right), m$ é constante, $m \ll T$ e (2.15) válido, então

$$
E\left[f_{x}^{T}(\lambda)\right]=f_{x}(\lambda)+O\left(T^{-1}\right)
$$

para $-\infty<\lambda<\infty$. Isto é,

$$
\lim _{T \rightarrow \infty} E\left[f_{x}^{T}(\lambda)\right]=f_{x}(\lambda) .
$$

Assim, $f_{x}^{T}(\lambda)$ é um estimador assintoticamente não viciado de $f_{x}(\lambda)$.

Teorema 2.7 Se $X_{i}, t=0, \pm 1, \ldots$, é uma série real estacionária, $\lambda-\frac{2 \pi s^{T}}{T}=O\left(T^{-1}\right)$, $\lambda \pm \mu \not \equiv 0(\bmod 2 \pi)$ e $m$ independe de $T$, então

$$
\operatorname{Var}\left[f_{x}^{T}(\lambda)\right]= \begin{cases}\frac{f_{x}^{2}(\lambda)}{2 m+1}+O\left(T^{-1}\right) & \text { se } \lambda \not \equiv 0(\bmod \pi) \\ \frac{f_{x}^{2}(\lambda)}{m}+O\left(T^{-1}\right) & \text { se } \lambda \equiv 0(\bmod \pi)\end{cases}
$$

e

$$
\operatorname{Cov}\left\{f_{x}^{T}(\lambda), f_{x}^{T}(\mu)\right\}=O\left(T^{-1}\right)
$$


Teorema 2.8 Sob as condições do Teorema 2.2 e seja $s^{T}$ um inteiro $\operatorname{com} \frac{2 \pi s^{T}}{T} \rightarrow \lambda$ quando $T \rightarrow \infty, \lambda_{j} \pm \lambda_{k} \not \equiv 0(\bmod 2 \pi)$ para $1 \leq j<k \leq J$. entào

(a) Para $j=1, \ldots, J, f_{x}^{T}\left(\lambda_{j}\right) \stackrel{D}{\rightarrow} f_{x}\left(\lambda_{j}\right) \frac{\frac{12}{4 m+2}}{4 m+2}$ independentes se $\lambda_{j} \not \equiv 0(\bmod \pi) \mathrm{e}$

(b) Para $j=1, \ldots, J, f_{x}\left(\lambda_{j}\right) \frac{\frac{2_{2}^{2}}{2 m}}{2 m}$ independentes se $\lambda_{j} \equiv 0(\bmod \pi)$.

Definição 2.12 O processo $\left\{X_{t}\right\}$ é chamado de proctsso Gaussiano (ou normal) se, para qualquer $n$ e qualquer conjunto conjunto $t_{1}, t_{2}, \ldots t_{n} \in T$, a distribuição conjunta de probabilidade de $\left\{X_{t_{1}} \ldots, X_{t_{n}}\right\}$ é normal multivarida.

Como a distribuição normal multivariada é completamente definida por suas médias, variâncias e covariâncias. temos que a estrutura probabilística do processo Gaussiano é completamente definida por sua "função do valor médio" $E\left[X_{t}\right]$ e sua "função de covariância" a $\operatorname{Cov}\left[X_{s}, X_{t}\right]$. Entào, se um processo Gaussiano é estacionário de segunda ordem ele também é um processo estritamente estacionário.

Definição 2.13 O processo $\left\{a_{t}, t=0 . \pm 1, \pm 2, \ldots\right\}$ é chamado de processo puramente estacionário ou processo ruído branco se este consiste de uma sequência de variáveis aleatórias com média zero, variância $\sigma^{2}$ e não correlacionadas, isto é, se $\forall s \neq t \operatorname{Cov}\left[X_{s}, X_{t}\right]=0$.

Definição 2.14 Filtro linear é uma operação que usa um conjunto de coeficientes fixados e conhecidos $a_{t}, t=0, \pm 1, \ldots$, para suavizar uma série de entrada estacionária $X_{t}$ produzindo uma série de saída ou série filtrada, $Y_{t}$, da forma

$$
Y_{t}=\sum_{s=-\infty}^{\infty} a_{s} X_{t-s}
$$

onde os coeficientes $a_{t}$ são chamados coletivamente de funçào resposta de impulso.

Para que (2.21) exista como um limite em média quadrática e a transformada de Fourier

$$
A(\lambda)=\sum_{t=-\infty}^{\infty} a_{t} \exp i \lambda t, \quad-\infty<\lambda<\infty
$$


denominada funçào de transferência do filtro. exista, a condiçào de regularidade

$$
\sum_{s=-\infty}^{\infty}\left|a_{s}\right|<\infty
$$

deve ser satisfeita.

Teorema 2.9 Seja $\gamma_{x}(k)$ a função de autocovariância da série estacionária $X_{t}$, a função de autocovariância da série filtrada $Y_{t}$ obtida em (2.21) é dada por

$$
\gamma_{y}(k)=\sum_{r=-\infty}^{\infty} \sum_{s=-\infty}^{\infty} a_{r} \cdot \gamma_{x}(k-r+s) a_{s}
$$

Se $X_{t}$ é um processo ruído branco com variância $\sigma_{x}^{2}$ temos

$$
\gamma_{y}(k)=\sigma_{x}^{2} \sum_{s=-\infty}^{\infty} a_{s+k} a_{s}
$$

Teorema 2.10 Seja $f_{x}(\lambda)$ a função de densidade espectral da série estacionária $X_{t}$ e $A(\lambda)$ dada pela Equação 2.22 , a função de densidade espectral da série filtrada $Y_{t}$, obtida por (2.21) é clada por

$$
f_{y}(\lambda)=|A(\lambda)|^{2} f_{x}(\lambda), \quad-\infty<\lambda<\infty
$$




\section{Capítulo 3}

\section{Modelos Lineares}

Este capítulo tem como objetivo principal. definir e apresentar as propriedades do processos $A R M A(p, q)$ e $A R I M A(p, d, q)$, que serão úteis para a compreensão da teoria dos próximos capítulos. Entretanto, antes de definir tais processos definiremos o processo linear geral que servirá de base para a definição do processo $A R . M A(p, q)$.

Um processo estocástico linear, $\left\{X_{t}\right\}$, pode ser representado de duas formas diferentes:

(a) Como uma saída de um filtro linear cuja entrada é um processo ruído branco, $\left\{a_{t}\right\}$ de média zero e variância $\sigma_{a}^{2}$, ou seja,

$$
\begin{aligned}
\tilde{X}_{t} & =a_{t}+\psi_{1} a_{t-1}+\psi_{2} a_{t-2}+\ldots \\
& =a_{t}+\sum_{j=1}^{\infty} \psi_{j} a_{t-j}
\end{aligned}
$$

onde $\hat{X}_{t}=X_{t}-\mu_{x}$ é o desvio do processo de alguma origem, ou de sua média $\left(E\left[X_{t}\right]\right)$, se o processo for estacionário. A estacionariedade do processo será garantida se $\sum_{j=0}^{\infty}\left|\psi_{j}\right|<\infty$.

(b) Sob determinadas condições, através de uma soma ponderada de valores passados de $\tilde{X}_{t}$ mais um ruído adicional $a_{t}$, ou seja,

$$
\begin{aligned}
\tilde{X}_{t} & =\pi_{1} \tilde{X}_{t-1}+\pi_{2} \tilde{X}_{t-2}+\ldots+a_{t} \\
& =\sum_{j=1}^{\infty} \pi_{j} \tilde{X}_{t-j}+a_{t}
\end{aligned}
$$

onde $\left\{a_{t}\right\}$ é um processo ruído branco de média zero e variância $\sigma_{a}^{2}$. Desse modo, essa representação pode ser interpretada como uma regressào das observaçòes passadas $\tilde{X}_{t-1}, \tilde{X}_{t-2}, \ldots$ 
em $\tilde{I}_{t}$

Podemos reescrever (3.1) e obter

$$
\tilde{X}_{t}=\left(1+\sum_{j=1}^{\infty} \psi_{j} B^{j}\right) a_{t}
$$

ou

$$
\tilde{X}_{t}=\psi(B) a_{t}
$$

onde $B$ é o operador de translação ao passado, ou seja, $B^{j} X_{t}=X_{t-j}$ e $\psi(B)=\sum_{j=0}^{\infty} \psi_{j} B^{j}$, $\operatorname{com} \iota_{0}=1$, é a funçào geradora dos pesos $\psi$ do filtro linear que relaciona $\tilde{X}_{t}$ a $a_{t}$.

Da mesma maneira. podemos reescrever (3.2) para obter

$$
\left(1-\sum_{j=1}^{\infty} \pi_{j} B^{j}\right) \tilde{X}_{t}=a_{t}
$$

ou

$$
\pi(B) \tilde{X}_{t}=a_{t}
$$

onde, $\pi(B)=1-\sum_{j=1}^{\infty} \pi_{j} B^{j}$ é a função geradora dos pesos $\pi$.

Existe uma relaçào entre $\pi(B)$ e $\psi(B)$, facilmente obtida multiplicando ambos os lados de (3.4) por $\psi(B)$, isto é, $\psi(B) \pi(B) \tilde{X}_{t}=\psi(B) a_{t}$. Por outro lado, $\tilde{X}_{t}=\psi(B) a_{t}$. Então, $\psi(B) \pi(B)=1$, ou seja,

$$
\pi(B)=\psi^{-1}(B)
$$

\section{Propriedades do processo linear estacionário:}

Seja $\left\{X_{t}\right\}$ um processo estacionário com representação dada por (3.1), então:

1. A funçào de autocovariância do processo é dada por

$$
\gamma_{x}(k)=\sigma_{a}^{2} \sum_{j=0}^{\infty} \psi_{j} \psi{ }_{j+k} .
$$


2. Em particular, para $k=0$ temos a variância do processo

$$
\gamma_{x}(0)=\sigma_{x}^{2}=\sigma_{a}^{2} \sum_{j=0}^{\infty} \psi_{j}^{2}
$$

3. O espectro do processo é dado por

$$
\begin{aligned}
f_{x}(\lambda) & =2 \sigma_{a}^{2} \psi\left(e^{-i \lambda}\right) \psi\left(e^{i \lambda}\right) \\
& =2 \sigma_{a}^{2}\left|\psi\left(e^{-i \lambda}\right)\right|^{2}, \quad 0 \leq \lambda \leq \pi
\end{aligned}
$$

\subsection{Modelos $A R M A(p, q)$}

Antes de definir o processo $f R M A(p . q)$ serào apresentadas duas definições que ajudarão a compreender melhor a construção de tal processo.

Definição 3.1 O processo autoregressivo de ordem $p$, $f R(p)$, é um caso especial da representação (3.2), onde apenas os primeiros pesos $\pi$ sào diferentes de zero. Este processo é escrito da seguinte forma

$$
\tilde{X}_{t}=\phi_{1} \tilde{X}_{t-1}+\phi_{2} \tilde{X}_{t-2}+\ldots+\phi_{p} \tilde{X}_{t-p}+a_{t}
$$

onde $\phi_{1}, \phi_{2}, \ldots, \phi_{p}$ representa um conjunto finito de parâmetros e $\left\{a_{t}\right\}$ é um processo ruído branco de média zero e variância $\sigma_{a}^{2}$. Outra forma de escrever o processo (3.8) é dada por

$$
\left(1-\phi_{1} B-\phi_{2} B^{2}-\ldots-\phi_{p} B^{p}\right) \check{X}_{t}^{r}=a_{t}
$$

ou

$$
\phi(B) \tilde{X}_{t}=a_{t} .
$$

onde $\phi(B)$ é chamado de operador autoregressivo.

Definição 3.2 O processo de médias móveis de ordem $q, M A(q)$, é um caso especial da representação (3.1), onde apenas os $q$ primeiros pesos $\psi$ são diferentes de zero. Este processo é escrito da seguinte forma

$$
\tilde{X}_{t}=a_{t}-\theta_{1} a_{t-1}-\theta_{2} a_{t-2}-\ldots-\theta_{q} a_{t-q}
$$


onde $\theta_{1}, \theta_{2}, \ldots, \theta_{q}$ representam o conjunto finito de parâmetros e $\left\{a_{t}\right\}$ é um processo ruído branco de média zero e variância $\sigma_{a}^{2}$.

Outra forma de representaçào de (3.10) é dacla por

$$
\check{X}_{t}=\left(1-\theta_{1} B-\theta_{2} B^{2}-\ldots-\theta_{q} B^{q}\right) a_{t}
$$

ou

$$
\tilde{X}_{t}=\theta(B) a_{t}
$$

onde $\theta(B)$ é chamado de operador de médias móveis.

Definição 3.3 O processo autoregressivo-médias móveis de ordem $(p, q), A R M A(p, q)$, é uma mistura dos processos autoregressivo de ordem $p$ e de médias móveis de ordem $q$. Este processo é escrito da seguinte forma

$$
\tilde{X}_{t}-\phi_{1} \tilde{X}_{t-1}-\ldots-\phi_{p} \tilde{Y}_{t-p}=a_{t}-\theta_{q} a_{t-1}-\ldots-\theta_{q} a_{t-q}
$$

ou

$$
\phi(B) \tilde{\mathrm{X}}_{t}=\theta(B) a_{t}
$$

\section{Propriedades:}

1. O processo $A R M A(p, q)$ será estacionário se, e somente se, as raízes da equação característica $\phi(B)=0$ caírem fora do círculo unitário.

2. O processo $A R M A(p, q)$ será invertível se, e somente se, as raizes da equação característica $\theta(B)=0$ caírem fora do círculo unitário.

3. A função de autocovariância do processo $A R M A(p, q)$ é dada por

$$
\begin{aligned}
\gamma_{x}(k)= & \phi_{1} \gamma_{x}(k-1)+\ldots+\phi_{p} \gamma_{x}(k-p)+\gamma_{x a}(k) \\
& -\theta_{1} \gamma_{x a}(k-1)-\ldots-\theta_{q} \gamma_{x a}(k-q)
\end{aligned}
$$

onde $\gamma_{x a}(k)$ é a função de covariância cruzada entre $X^{r}$ e $a, \gamma_{x a}(k)=E\left[\tilde{X}_{t-k} a_{t}\right]$, dada pela Definição 2.8. Como $X_{t-k}$ depende apenas dos ruídos que ocorreram até o tempo $t-k$, temos

$$
\begin{array}{ll}
\gamma_{x a}(k)=0 & k>0 \\
\gamma_{x a}(k) \neq 0 & k \leq 0
\end{array}
$$


Com isso, temos

$$
\hat{i x}_{x}(k)=\phi_{1} \hat{\imath} x(k-1)+\ldots+o_{p} \hat{i} x_{x}(k-p), \quad k \geq q+1
$$

4. Pelo item anterior, temos

$$
\rho_{x}(k)=\phi_{1} \rho_{x}(k-1)+\ldots+\phi_{p} \rho_{x}(k-p), \quad k \geq q+1
$$

oul

$$
o(B) \rho_{x}(k)=0, \quad k \geq q+1 .
$$

5. Para $k=0$ temos a variância do processo $A R M A(p, q)$

$$
\begin{aligned}
\gamma_{x}(0)= & o_{1 \gamma_{x}}(1)+\ldots+\phi_{p} \gamma_{x}(p)+\sigma_{a}^{2} \\
& -\theta_{1} \gamma_{x a}(-1)-\ldots-\theta_{\eta} \gamma_{x a}(-q)
\end{aligned}
$$

6. O espectro do processo $A R . M . A(p, q)$ é dado por

$$
\begin{aligned}
f_{x}(\lambda) & =\frac{\sigma_{a}^{2}}{2 \pi} \frac{\left|\theta\left(e^{-i \lambda}\right)\right|^{2}}{\left|\phi\left(e^{-i \lambda}\right)\right|^{2}} \\
& =\frac{\sigma_{a}^{2}}{2 \pi} \frac{\left|1-\theta_{1} e^{-i \lambda}-\ldots-\theta_{q} e^{-i \lambda q}\right|^{2}}{\left|1-o_{1} e^{-i \lambda}-\ldots-\phi_{p} e^{-i \lambda p}\right|^{2}}, \quad 0 \leq \lambda \leq \pi
\end{aligned}
$$

\subsection{Modelos ARIMA(p,d.q)}

Sabemos que um processo $A R M A(p, q)$ é estacionário se as raízes da equação característica $\phi(B)=0$ caem fora do círculo unitário e que não é estacionário caso contrário. O modelo $A R I M A(p, d, q)$ é útil para modelar os processos em que uma ou mais raízes dessa equação caem sobre o círculo unitário, ou seja, valem um. Neste caso, o processo será chamado de processo nào estacionário homogêneo.

Considere o modelo

$$
\varphi(B) \tilde{X}_{t}=\theta(B) a_{t}
$$

onde $\varphi(B)$ é um operador nào estacionário. tal que $d$ das raízes de $\varphi(B)=0$ valem um e as demais caem fora do círculo unitário. Então, podemos representar o modelo (3.19) da seguinte forma

$$
\varphi(B) \check{X}_{t}=o(B) \nabla^{d} \dot{X}_{t}=\theta(B) a_{t}
$$


onde $\nabla^{d}=(1-B)^{d}$ é chamado de operador diferença de ordem d. $\theta(B)$ e $\theta(B)$ são os operadores autoregressivo e de médias móveis. respectivamente. definidos na seção anterior.

Definição 3.4 O processo

$$
\phi(B) \nabla^{d} \dot{\mathrm{X}}_{t}=\theta(B) a_{t},
$$

onde

$$
\begin{aligned}
& \phi(B)=1-\phi_{1} B-\dot{\phi}_{2} B^{2}-\ldots-\phi_{p} B^{p} \\
& \theta(B)=1-\theta_{1} B-\theta_{2} B^{2}-\ldots-\theta_{q} B^{q},
\end{aligned}
$$

é chamado de processo autoregressivo integrado de médias móveis ou $A R I M A(p, d, q)$.

De maneira equivalente, o processo $f R I . M A(p . d, q)$ pode ser definido pelas duas equações abaixo

$$
\begin{aligned}
\phi(B) W_{t} & =\theta(B) a_{t} \\
W_{t} & =\nabla^{d} \check{X}_{t} .
\end{aligned}
$$

Podemos ver que esse modelo assume que a $d$-ésima diferença da série pode ser representada por um processo ARMA estacionário e invertível. Ou seja, assume-se que:

(a) $\phi(B)$ é um operador autoregressivo estacionário, ou seja, as raízes da equação $\phi(B)=0$ caem fora do círculo unitário,

(b) $\varphi(B)$, chamado de operador autoregressivo generalizado, é um operador não estacionário com d das raízes da equação $\varphi(B)=0$ iguais a um e as demais caem fora do círculo unitário,

(c) $\theta(B)$, chamado de operador de médias móveis, é um operador invertível, ou seja, as raízes da equação $\theta(B)=0$ caem fora do círculo unitário.

Existem três formas explícitas de representar o modelo $A R I M A(p, d, q)$, que são consideradas a seguir. 
Forma de equações de diferenças

Usando diretamente equações de diferenças podemos expressar o valor atual do processo $\tilde{X}_{t}$ em função de valores passados dos $\tilde{X}$ 's e dos valores atual e passados dos $a^{\prime} s$. Isto é, se

$$
\varphi(B)=\phi(B)(1-B)^{d}=1-\varphi_{1} B-\varphi_{2} B^{2}-\ldots-\varphi_{p+d} B^{p+d}
$$

o modelo (3.20) pode ser escrito como

$$
\tilde{X}_{t}=\varphi_{1} \tilde{X}_{t-1}+\ldots+\varphi_{p+d} \tilde{X}_{t-p-d}-\theta_{1} a_{t-1}-\ldots-\theta_{q} a_{t-q}+a_{t}
$$

\section{Forma de choques aleatórios}

Neste caso. o modelo representa o processo $\tilde{\mathrm{X}}_{t}$ como uma saída de um filtro linear cuja entrada são ruídos brancos, ou uma sequência de choques nào correlacionados, $a_{t}$. Ou seja, o modelo é dado por

$$
\begin{aligned}
\tilde{X}_{t} & =a_{t}+\psi_{1} a_{t-1}+\psi_{2} a_{t-2}+\ldots \\
& =\psi(B) a_{t}
\end{aligned}
$$

Se multiplicarmos ambos os lados de (3.24) pelo operador $\varphi(B)$, obtemos

$$
\varphi(B) \tilde{X}_{t}=\varphi(B) \psi(B) a_{t}
$$

Entretanto, como

$$
\varphi(B) \tilde{X}_{t}=\theta(B) a_{t}
$$

temos que

$$
\varphi(B) \psi(B)=\theta(B)
$$

Portanto, os pesos $\psi$ podem ser obtidos equacionando-se os coeficientes de $B$ na expansão

$$
\left(1-\varphi_{1} B-\ldots-\varphi_{p+d} B^{p+d}\right)\left(1+\psi_{1} B+\psi_{2} B^{2}+\ldots\right)=\left(1-\theta_{1} B-\ldots-\theta_{q} B^{q}\right)
$$




\section{Forma invertida}

Neste caso, o modelo representa o processo $\dot{X}_{t}$ em termos de valores passados dos $\tilde{X}^{\prime} s$ e do valor atual do ruído $a_{t}$. Considere o modelo

$$
\check{X}_{t}=\psi(B) a_{t}
$$

que pode ser escrito na forma invertida

$$
\psi^{-1}(B) \tilde{X}_{t}=a_{t}
$$

ou

$$
\pi(B) \tilde{\mathrm{X}}_{t}=\left(1-\sum_{j=1}^{\infty} \pi_{j} B^{j}\right) \tilde{\mathrm{X}}_{t}=a_{t} .
$$

Isto é, $\tilde{X}_{t}$ é uma soma ponderada infinita dos valores passados de $\tilde{X}$, mais um ruído presente,

$$
\dot{X}_{t}=\pi_{1} \dot{X}_{t-1}+\pi_{2} \tilde{X}_{t-2}+\ldots+a_{t}
$$

O modelo é dito invertível se os pesos $\pi$ formarem uma série absolutamente convergente, ou seja, $\pi(B)$ deve convergir sobre ou dentro do círculo unitário.

Substituindo (3.25) em

$$
\varphi(B) \tilde{X}_{t}=\theta(B) a_{t}
$$

obtemos

$$
\varphi(B) \tilde{X}_{t}=\theta(B) \pi(B) \tilde{X}_{t}
$$

Então, os pesos $\pi$ serão obtidos equacionando os coeficientes de $B \mathrm{em}$

$$
\varphi(B)=\theta(B) \pi(B)
$$

ou seja, em

$$
\left(1-\varphi_{1} B-\ldots-\varphi_{p+d} B^{p+d}\right)=\left(1-\theta_{1} B-\ldots-\theta_{q} B^{q}\right)\left(1-\pi_{1} B-\pi_{2} B^{2}-\ldots\right) .
$$




\section{Capítulo 4}

\section{Modelos de Longa Memória}

\subsection{Introdução}

Estudos empíricos, principalmente em Economia e Hidrologia, revelam séries temporais que apresentam uma dependência significativa entre observações distanciadas por um longo intervalo de tempo. Essas séries são denominadas de séries de longa memória e não são bem ajustadas por modelos que têm como suposição a não significância das correlações entre observações distantes. ou seja, que têm como suposiçào a independência assintótica. Com a constatação desse problema, iniciou-se um estudo das características dessas séries para que se pudesse encontrar uma ou mais famílias de modelos capazes de representar adequadamente esse comportamento de longa memória. Verificou-se que uma das principais características desse tipo de série é que sua densidade espectral é nào limitada na frequência zero, o que equivale dizer que sua função de autocorrelaçào não é absolutamente somável. Outra característica é o decaimento hiperbólico da função de autocorrelação desse tipo de série.

Procurando respeitar as características de uma série de longa memória citadas acima, foram definidos dois modelos importantes, nos quais a funçào de densidade espectral é proporcional a $\lambda^{-r}, 1<r<2$, para $\lambda$ próximo de zero, e o decaimento da função de autocorrelaçào é proporcional a $\tau^{r-1}$. Primeiro foi introduzido o modelo de ruído Gaussiano fracionário por Mandelbrot \& Ness (1968). Mais tarde Granger \& Joyeux (1980) e Hosking (1981) introduziram o modelo ARIMA fracionário (ou ARFIMA) que é uma generalização 
do modelo ARIMA. Geweke \& Porter-Hudak (1983) mostraram a equivalência desses dois modelos.

Mandelbrot \& Van Ness (1968). Mandelbrot \& Wallis (1968, 1969) estudaram em detalhes algumas famílias de funçòes aleatórias que poderiam, de alguma maneira, representar o comportamento típico do processo que não possui independência assintótica. Deste modo, estudaram o movimento Browniano fracionário a fim de encontrar resultados de interesse prático e introduziram o modelo que chamamos de modelo de ruído Gaussiano fracionário. A seguir, serão apresentadas algumas definições necessárias para a introdução deste modelo.

Definição 4.1 O movimento Browniano ordinário, $B(t . \omega)$ de Bachelier, Wiener \& Lévy, $-\infty<t<\infty . \omega \in \Omega$. é uma funçào aleatória real de incrementos (iaussianos independentes tal que $B\left(t_{2}, \omega\right)-B\left(t_{1}, \omega^{\prime}\right)$ têm média zero e variância $\left|t_{2}-t_{1}\right|$, e tal que $B\left(t_{2}, \omega\right)-B\left(t_{1}, \omega\right)$ sào independentes de $B\left(t_{4}, \omega\right)-B\left(t_{3}, \omega\right)$ se os intervalos $\left(t_{1}, t_{2}\right)$ e $\left(t_{3}, t_{4}\right)$ não se sobrepõem.

Notação: para simplificar denotaremos $B(t, \omega)$ por $B(t)$.

Definição 4.2 Seja $B(t)$ um movimento Browniano ordinário e $H$ um parâmetro que satisfaz $0<H<1$. O movimento Browniano fracionário de expoente $H, B_{H}(t)$, é uma média móvel da derivada de $B(t), d B(t)$, no qual incrementos passados de $B(t)$ são ponderados pela semente $(t-s)^{H-1 / 2}$, ou seja,

$$
B_{H}(t)=\frac{1}{\Gamma\left(H+\frac{1}{2}\right)} \int_{-\infty}^{t}(t-s)^{H-1 / 2} d B(s),
$$

A seguir, apresentaremos algumas propriedades do movimento Browniano fracionário demonstradas por Mandelbrot \& Van Ness (1968).

\section{Propriedades:}

1. o movimento Browniano fracionário de parâmetro $\mathrm{H}$ é a $\left(\frac{1}{2}-H\right)$-ésima derivada fracionária do movimento Browniano;

2. a densidade espectral do movimento Browniano fracionário é proporcional a $\lambda^{-2 H-1}$;

3. a função de covariância do movimento Browniano fracionário é proporcional a $|k|^{2 H-2}$. 
Definição 4.3 O processo de ruído fracionário no tempo contínuo é a derivada do movimento Browniano fracionário. $B_{H}^{\prime}(t)$.

Um análogo, no tempo discreto, do processo ruído fracionário no tempo contínuo é dado pela definição abaixo

Definição $4.4 X_{t}=B_{H}(t)-B_{H}(t-1) t=0,1, \ldots$ é denominado ruído Gaussiano fracionário.

Propriedades: (Geweke \& Porter-Hudak (1983))

1. A densidade espectral de $\left\{X_{t}\right\}$ é dada por:

$$
\begin{aligned}
f_{1}(\lambda: H)= & \sigma^{2}(2 \pi)^{-2 H-2} \Gamma(2 H+1) \operatorname{sen}(\pi H) 4 \operatorname{sen}^{2}(\lambda / 2) \\
& \sum_{n=-\infty}^{\infty}|n+(\lambda / 2 \pi)|^{-2 H-1}
\end{aligned}
$$

onde

$$
\Gamma(z):= \begin{cases}\int_{0}^{\infty} t^{z-1} e^{-t} d t, & z>0, \\ \infty, & z=0, \\ \tilde{\sim}^{-1} \Gamma(1+z), & z<0 .\end{cases}
$$

2. $\lim _{\lambda \rightarrow 0} \lambda^{2 H-1} f_{1}(\lambda ; H)=\left(2 \sigma^{2} / \pi\right) \Gamma(2 H+1) \operatorname{sen}(\pi H)$.

3. A função de autocorrelação correspondente é dada por

$$
\rho_{1}(\tau ; H)=0.5\left(|\tau-1|^{2 H}-2|\tau|^{2 H}+|\tau+1|^{2 H}\right) .
$$

4. A expansão em série de Taylor de $4.3 \mathrm{em}$ torno de $(\tau-1) / \tau$ e $(\tau+1) / \tau$ mostra que

$$
\lim _{\tau \rightarrow \infty} \tau^{2-2 H} \rho_{1}(\tau: H)=H(2 H-1) .
$$

5. Pela desigualdade de Schwarz, $\rho_{1}(\tau ; H)>0(<0)$ para $\tau \neq 0$ se $H>0.5(<0.5)$. 
Definição $4.5 \mathrm{Um}$ processo $\left\{X_{t}, t=0.1 \ldots\right\}$ é chamado de ruído Gaussiano fracionário geral se sua densidade espectral é da forma $f_{1}(\lambda: H) f_{u}(\lambda)$ onde $f_{u}(\lambda)$ é uma função contínua positiva limitada superiormente em qualquer intervalo fechado que nào contém zero ("bounded above and away from zero") no intervalo $[-\pi, \pi]$.

\subsection{Modelo ARIMA Fracionário (ARFIMA)}

Um processo no tempo discreto, análogo ao movimento Browniano, é o passeio aleatório ou o processo $\operatorname{ARIMA}(0,1,0), X_{t}=X_{t-1}+a_{t}$, onde $a_{t}$ são variáveis aleatórias não correlacionadas e identicamente distribuídas. Neste caso. a primeira diferença de $\left\{X_{t}\right\}$ é o processo ruido branco no tempo discreto, $\left\{a_{t}\right\}$.

Observação: A partir deste momento assumiremos que $E\left(X_{t}\right)=0$.

Definição 4.6 O operador de diferença fracionária é dado por

$$
\begin{aligned}
\nabla^{d} & =(1-B)^{d}=\sum_{k=0}^{\infty}\left(\begin{array}{l}
d \\
k
\end{array}\right)(-B)^{k}=1-d B-\frac{1}{2 !} d(1-d) B^{2}- \\
& -\frac{1}{3 !} d(1-d)(2-d) B^{3}-\ldots
\end{aligned}
$$

Definição 4.7 O processo ruído branco de diferença fracionária, $X_{t}$, com parâmetro $H$, $0<H<1$ é a $\left(\frac{1}{2}-H\right)$-ésima diferença fracionária do processo ruído branco no tempo discreto. Ou seja, $X_{t}=\nabla^{-d} a_{t}$ ou $\nabla^{d} X_{t}=a_{t}, \operatorname{com} d=H-\frac{1}{2}$, onde $a_{t}$ é um processo ruído branco de média zero e varância $\sigma_{a}^{2}$.

\section{Observações:}

(a) Fazendo $d=H-\frac{1}{2}$, o processo ruído branco de diferença fracionária $\left\{X_{t}\right\}$ é análogo ao processo ruído fracionário no tempo contínuo com parâmetro $\mathrm{H}$, dado pela Definição 4.3.

(b) O proceso $X_{t}$, dado pela Definiçào 4.7, é equivalente ao processo $\operatorname{ARIMA}(0, d, 0)$ com $|d|<1 / 2$, que será denominado processo ARFIMA(0.d,0).

O teorema abaixo enuncia algumas propriedades do processo $\left\{X_{t}\right\}$. 
Teorema 4.1 Seja $\left\{X_{t}\right\}$ um processo $f R F I M A(0, d, 0)$.

(a) Quando $d<\frac{1}{2},\left\{X_{t}\right\}$ é um processo estacionário e tem representação de médias móveis infinita

$$
X_{t}=\psi(B) a_{t}=\sum_{k=0}^{\infty} \psi_{k} a_{t-k}
$$

onde

$$
\psi_{k}=\frac{d(1+d) \ldots(k-1+d)}{k !}=\frac{(k+d-1) !}{k !(d-1) !},
$$

$\operatorname{com} \psi_{k} \sim k^{d-1} /(d-1)$ ! quando $k \rightarrow \infty$.

(b) Quando $d>-\frac{1}{2} .\left\{X_{t}\right\}$ é invertível e tem representaçào autoregressiva infinita

$$
\pi(B) X_{t}=\sum_{k=0}^{\infty} \pi_{k} X_{t-k}=a_{t}
$$

onde

$$
\pi_{k}=\frac{-d(1-d) \ldots(k-1-d)}{k !}=\frac{(k-d-1) !}{k !(-d-1) !}
$$

$\operatorname{com} \pi_{k} \sim k^{-d-1} /(-d-1)$ ! quando $k \rightarrow \infty$.

Nos próximos ítens assumiremos que $-\frac{1}{2}<d<\frac{1}{2}$.

(c) A densidade espectral de $\left\{X_{t}\right\}$ é dada por

$$
f(\lambda)=\frac{\sigma_{a}^{2}}{2 \pi}\left(2 \operatorname{sen} \frac{1}{2} \lambda\right)^{-2 d}
$$

para $0<\lambda \leq \pi$ e $f(\lambda) \sim \lambda^{-2 d}$ quando $\lambda \rightarrow 0$.

(d) A funçào de autocovariância de $\left\{X_{t}\right\}$ é dada por

$$
\gamma_{k}=E\left(X_{t} X_{t-k}\right)=\frac{(-1)^{k}(-2 d) !}{(k-d) !(-k-d) !} \sigma_{a}^{2}
$$

e a funçào de autocorrelação de $\left\{X_{t}\right\}$ é dada por

$$
\rho_{k}=\gamma_{k} / \gamma_{0}=\frac{(-d) !(k+d-1) !}{(d-1) !(k-d) !} \quad(k=0, \pm 1, \ldots)
$$




$$
=\frac{(d)(1+d) \ldots(k-1+d)}{(1-d)(2-d) \ldots(k-d)} \quad(k=1,2 \ldots) .
$$

Em particular, $\gamma_{0}=(-2 d) ! /(-d) !^{2}$ e $\rho_{1}=d /(1-d)$. Quando $k \rightarrow \infty$,

$$
\rho_{k} \sim \frac{(-d) !}{(d-1)} k^{2 d-1}
$$

(e) A função de autocorrelação inversa de $\left\{X_{t}\right\}$, quando, $k \rightarrow \infty$, é dada por

$$
\rho_{i n v, k}=\frac{d !(k-d-1) !}{(-d-1) !(k+d) !} \sim \frac{d !}{(-d-1) !} k^{-1-2 d} .
$$

(f) As autocorrelaçòes parciais de $\left\{X_{t}\right\}$ sào dadas por

$$
\phi_{k k}=d /(k-d)(k=1,2 \ldots) .
$$

Demonstraçào: Para maiores detalhes ver Hosking (1981).

Pelo Teorema 4.1 sabemos que se $-\frac{1}{2}<d<\frac{1}{2}$ o processo $\left\{X_{t}\right\}$ é estacionário e invertível, além disso, $\psi_{k}$ e $\pi_{k}$ decaem hiperbolicamente. O comportamento do espectro nas frequências baixas indica que, para $d>0,\left\{X_{t}\right\}$ é um processo de longa memória, que também pode ser caracterizado pelo decaimento hiperbólico da função de autocorrelação.

Observação: No proceso $A R F I M A(0, d, 0)$ o parâmetro $d$ pode assumir qualquer valor real. Neste caso, o processo pode ser diferenciado um número finito de vezes até que $|d|<$ 1 e este será estacionário e invertível se $|d|<1 / 2$. Ou seja, $\nabla^{n}\left\{X_{t}\right\}$ será um processo $A R F I M A(0, d, 0)$ estacionário e invertível se $\nabla^{d} \nabla^{n} X_{t}=a_{t}$, onde $|d|<1 / 2$ e $n \in I N$.

A seguir, analisaremos algumas características do processo $A R F I M A(0, d, 0)$ com respeito aos valores que $d$ assume no intervalo $\left[-\frac{1}{2}, \frac{1}{2}\right]$. Os principais resultados são:

(a) $d=\frac{1}{2}$, a densidade espectral do processo $A R F I M . A\left(0, \frac{1}{2}, 0\right)$ é dada por

$$
f(\lambda)=\frac{1}{2 \operatorname{sen}\left(\frac{1}{2} \lambda\right)} \sim \lambda^{-1}
$$

quando $\lambda \rightarrow 0$. 
A representaçào formal de médias móveis infinita desse processo tem coeficientes $\psi_{k} \sim(n k)^{-\frac{1}{2}}$ quando $k \rightarrow \infty$. Esse fato indica que $\sum_{k} \psi_{k}^{2}$ diverge e. portanto, $\left\{X_{t}\right\}$ é não estacionário. Entretanto, $\left\{X_{t}\right\}$ é invertivel, pois

$$
\left(1-\frac{1}{2} B-\frac{1}{8} B^{2}-\frac{1}{16} B^{3}-\frac{5}{128} B^{4}-\ldots\right) x_{t}=a_{t},
$$

o que indica $\sum_{k=0}^{\infty}\left|\pi_{k}\right|<\infty$.

(b) $0<d<\frac{1}{2}$, neste caso, o processo $A R F I M A(0, d, 0)$ é um processo estacionário de longa memória. As autocorrelaçòes e autocorrelaçòes parciais de $\left\{X_{t}\right\}$ são todas positivas e decaem monotonicamente e hiperbolicamente para zero e $\sum_{k=-\infty}^{\infty}\left|\rho_{k}\right|=\infty$. A densidade espectral de $\left\{X_{t}\right\}$ é concentrada em frequências baixas: $f(\lambda)$ é uma função decrescente de $\lambda$ e $f(\lambda) \rightarrow \infty$ quando $\lambda \rightarrow 0$, mas é integrável.

(c) $\mathrm{d}=0$, o processo $A R F I M A(0,0,0)$ é ruído branco, que tem autocorrelações zero e densidade espectral constante.

(d) $-\frac{1}{2}<d<0$, o processo $\operatorname{ARFIMA}(0, d, 0)$ tem memória intermediária. As autocorrelações e autocorrelações parciais do processo são todas negativas, exceto $\rho_{0}=1$, e decaem monotonicamente e hiperbolicamente para zero e $\sum_{k=-\infty}^{\infty}\left|\rho_{k}\right|<\infty$. A densidade espectral é dominada por componentes de alta frequência; $f(\lambda)$ é uma função crescente de $\lambda, f(\lambda)=0$ quando $\lambda \rightarrow 0$, embora tenha gradiente $+\infty$.

(e) $d=-\frac{1}{2}$, o processo $A R F I M A\left(0,-\frac{1}{2}, 0\right)$ é estacionário, mas nào é invertível. Os pesos $\psi_{k}$ da representação infinita de médias móveis são os mesmos que os pesos $\pi_{k}$ de um processo ARF IMA $\left(0, \frac{1}{2}, 0\right)$ e, portanto, decaem como $k^{-3 / 2}$ para $k$ grande. A densidade espectral do processo é dada por $f(\lambda)=2 \operatorname{sen}\left(\frac{1}{2} \lambda\right)$ que tende para zero quando $\lambda \rightarrow 0$, mas tem gradiente finito +1 em $\lambda=0$. A função de autocorrelação do processo é dada por $\rho_{k}=-1 /\left(4 k^{2}-1\right)$; a variância do processo é $\gamma_{0}=4 / \pi$. As autocorrelaçòes parciais do processo são dadas por $\phi_{k k}=-1 /(2 k+1)$.

Algumas vezes, utilizar o modelo $A R F I M A(0, d, 0)$ em séries com comportamento de 
longa memória pode não ser satisfatório, pois algumas destas séries também podem apresentar um comportamento de curta memória. Entào. o ideal é encontrar um modelo que seja capaz de explicar o comportamento de curta memória e que mantenha o decaimento hiperbólico da função de autocorrelação, característico do processo de longa memória. Uma extensão natural do processo $\operatorname{ARFIMA}(0, d, 0)$ que possui essas propriedades é o processo que combina diferença fracionária com a família de modelos de Box \& Jenkins. Tal processo é definido a seguir.

Definição $4.8\left\{Y_{t}\right\}$ é um processo $A R F I M A(p, d, q) \operatorname{com} d \in\left(-\frac{1}{2}, \frac{1}{2}\right)$ se $\left\{Y_{t}\right\}$ é estacionário e satisfaz a equação,

$$
\phi(B) \nabla^{d} Y_{t}=\theta(B) a_{t}
$$

onde $\left\{a_{t}\right\}$ é ruído branco e $\phi$ e $\theta$ são polinômios em $\mathrm{B}$ de graus $p$ e $q$, respectivamente.

A razão da escolha dessa família de processos, para fins de modelagem das séries com comportamento de longa memória, é que o efeito do parâmetro $d$ em observações distantes decai hiperbolicamente conforme a distância aumenta, enquanto os efeitos dos parâmetros $\phi$ e $\theta$ decaem exponencialmente. Então, $d$ deve ser escolhido com o objetivo de explicar a estrutura de correlação de ordens altas da série, enquanto os parâmetros $\phi$ e $\theta$ explicam a estrutura de correlação de ordens baixas.

Observação: É esperado que o comportamento de longa memória de um processo ARFIMA $(p, d, q)$ seja semelhante ao do processo $A R F I M A(0, d, 0)$ com o mesmo valor de $d$, desde que, para observações muito distantes o efeito dos parâmetros $\phi$ e $\theta$ não seja significativo.

Teorema 4.2 Seja $\left\{Y_{t}\right\}$ um processo $A R F I M A(p, d, q)$. Então,

(a) $\left\{Y_{t}\right\}$ é estacionário se $d<\frac{1}{2}$ e todas as raízes da equação $\phi(z)=0$ estão fora do círculo unitário;

(b) $\left\{Y_{t}\right\}$ é invertível se $d>-\frac{1}{2}$ e todas as raízes da equação $\theta(z)=0$ estão fora do círculo unitário. 
Se $\left\{Y_{t}\right\}$ é estacionário e invertível, com função de densidade espectral $f(\lambda)$ e função de autocorrelação $\rho_{k}$, entào

(c) $\lim _{\lambda \rightarrow 0} \lambda^{2 d} f(\lambda)$ existe e é finito;

(d) $\lim _{k \rightarrow \infty} k^{1-2 d} \rho_{k}$ existe e é finito.

Demonstração: Para maiores detalhes ver Hosking (1981).

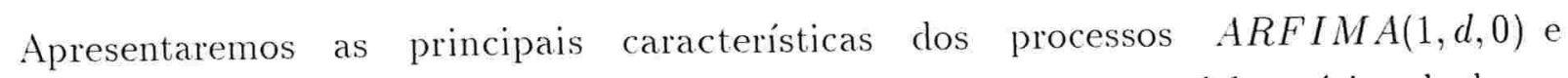
$A R F I M A(0, d, 1)$ que. na prática, sào os mais utilizados para modelar séries de longa memória.

O processo $A R F I M A(1, d, 0)$ é dado por $(1-\phi B) \nabla^{d} Y_{t}=a_{t}$, onde $\left\{a_{t}\right\}$ é um processo ruído branco. Escrevemos $X_{t}=(1-\phi B) Y_{t}$, assim, temos $\nabla^{d} X_{t}=a_{t}$. Então, $\left\{Y_{t}\right\}$ é uma autoregressão de primeira ordem com ruídos gerados pelo processo $A R I M A(0, d, 0)$. Para assegurar estacionariedade e invertibilidade de $\left\{Y_{t}\right\}$ assumimos que $|\phi|<1$ e $|d|<\frac{1}{2}$.

As autocorrelações de $\left\{Y_{t}\right\}$ são expressadas mais convenientemente se utilizarmos a função hipergeométrica

$$
F(a, b ; c ; z)=1+\frac{a b}{c .1} z+\frac{a(a+1) b(b+1)}{c(c+1) 1.1} z^{2}+\ldots
$$

Pelo Teorema 4.2 temos os seguintes resultados.

Teorema 4.3 ( Hosking (1981)) Sejam $\left\{Y_{t}\right\}$ um processo $A R F I M A(1, d, 0)$ estacionário e invertivel e $X_{t}=(1-\phi B) Y_{t}$.

(a) Os pesos $\psi$ e $\pi$ das representações autoregressiva e de médias móveis infinitas de $\left\{Y_{t}\right\}$ são dados por

$$
\psi_{k}=\frac{(k+d-1) !}{k !(d-1) !} F(1,-k ; 1-d-k ; \phi) \sim \frac{k^{d-1}}{(1-\phi)(d-1) !},
$$




$$
\pi_{k}=\frac{(k-d-2) !}{(k-1)(-d-1) !}\{1-\phi-(1+d) / k\} \sim \frac{(1-o)}{(-d-1) !} k^{-d-1}
$$

quando $k \rightarrow \infty$.

(b) A densidade espectral de $\left\{Y_{t}\right\}$ é

$$
f(\lambda)=\frac{\left\{2 \operatorname{sen}\left(\frac{1}{2} \lambda\right)\right\}^{-2 d}}{1+\phi^{2}-2 \phi \cos \lambda} \sim \frac{\lambda^{-2 d}}{(1-0)^{2}}
$$

quando $\lambda \rightarrow 0$.

(c) Sejam $\gamma_{k}^{y}, \rho_{k}^{y}, \gamma_{k}^{x} \cdot \rho_{k}^{x}$ as autocovariâncias e as autocorrelaçòes de $\left\{Y_{t}\right\}$ e $\left\{X_{t}\right\}$ respectivamente, onde $\gamma_{k}^{x}$ e $\rho_{k}^{x}$ são dadas em (4.13) e (4.11), respectivamente. então

$$
\begin{gathered}
\gamma_{k}^{y}=\gamma_{k}^{x}\{F(1, d+k ; 1-d+k ; \phi)+F(1, d-k ; 1-d-k ; \phi)-1\} /\left(1-\phi^{2}\right), \\
\rho_{k}^{y}=\rho_{k}^{x} \frac{\{F(1, d+k ; 1-d+k ; \phi)+F(1, d-k ; 1-d-k: \phi)-1\}}{(1-\phi) F(1,1+d ; 1-d-k ; \phi)} .
\end{gathered}
$$

Em particular,

$$
\begin{aligned}
\gamma_{0}^{y} & =\frac{(-2 d) !}{\{(-d) !\}^{2}} \frac{F(1,1+d ; 1-d ; \phi)}{(1+\phi)} . \\
\rho_{1}^{y} & =\frac{\left(1+\phi^{2}\right) F(1, d ; 1-d ; \emptyset)-1}{\phi\{2 F(1, d ; 1-d ; \phi)-1\}} .
\end{aligned}
$$

quando $k \rightarrow \infty$,

$$
\rho_{k}^{y} \sim \frac{(-d) !}{(d-1) !} \frac{(1+\phi)}{(1-\phi)^{2}} \frac{k^{2 d-1}}{F(1,1+d: 1-d: \phi)}
$$

O processo $A R I M A(0, d, 1), Y_{t}=(1-\theta B) \nabla^{-d} a_{t}$. onde $\left\{a_{t}\right\}$ é um processo ruído branco, pode ser descrito como uma média móvel de primeira ordem de um ruído branco com diferença fracionária. $\left\{Y_{t}\right\}$ é estacionário e invertível para $|\theta|<1$ e $|d|<\frac{1}{2}$.

Pelo Teorema 4.2 temos os seguintes resultados. 
Teorema 4.4 (Hosking (1981)) Seja $\left\{Y_{t}\right\}$ um processo $\mathcal{A} F F I . \mathcal{A}(0, d, 1)$ estacionário e invertivel: seja $\nabla^{d} X_{t}=a_{t}$ tal ciue $Y_{t}=(1-\theta B) X_{t}$.

(a) Os pesos 4 , e $\pi$ das representaçòes autoregressiva e de médias móveis infinitas de $\left\{Y_{t}\right\}$ são dados por

$$
\begin{aligned}
\psi_{k}= & \frac{(k-d-1) !}{k !(-d-1) !} F(1,-k ; 1+d-k ; \theta) \sim \frac{k^{-d-1}}{(1-\theta)(-d-1) !}, \\
\pi_{k}= & \frac{(k+d-2) !}{(k-1)(+d-1) !}\{1-\theta-(1+d) / k\} \sim \frac{(1-\theta)}{(d-1) !} k^{d-1},
\end{aligned}
$$

quando $k \rightarrow \infty$.

(b) A densidade espectral de $\left\{Y_{t}\right\}$ é

$$
f(\lambda)=\left(1+\theta^{2}-2 \theta \cos \lambda\right)\left\{2 \operatorname{sen}\left(\frac{1}{2} \lambda\right)\right\}^{-2 d} \sim(1-\theta)^{2} \lambda^{-2 d}
$$

quando $\lambda \rightarrow 0$.

(c) Sejam $\gamma_{k}^{y}, \rho_{k}^{y}, \gamma_{k}^{x}, \rho_{k}^{x}$ as autocovariâncias e as autocorrelaçòes de $\left\{Y_{t}\right\}$ e $\left\{X_{t}\right\}$ respectivamente, onde $\gamma_{k}^{r}$ e $\rho_{k}^{x}$ sào dadas em (4.13) e (4.11), respectivamente, então

$$
\begin{gathered}
\gamma_{k}^{y}=\gamma_{k}^{x} \frac{(1-\theta)^{2} k^{2}-(1-d)\left\{(1-d)\left(1+\theta^{2}\right)-2 \theta d\right\}}{k^{2}-(1-d)^{2}}, \\
\rho_{k}^{y}=\rho_{k}^{x} \frac{a k^{2}-(1-d)^{2}}{k^{2}-(1-d)^{2}},
\end{gathered}
$$

onde $a=(1-\theta)^{2} /\left\{1+\theta^{2}-2 \theta d /(1-d)\right\}$. Em particular,

$$
\begin{gathered}
\gamma_{0}^{y}=\left\{1+\theta^{2}-2 \theta d /(1-d)\right\}(-2 d) ! /\{(-d) !\}^{2}, \\
\rho_{1}^{y}=\frac{a-(1-d)^{2}}{(1-d)(2-d)}=\frac{\left(1+\theta^{2}\right) d(2-d)-2 \theta\left(1-d+d^{2}\right)}{(1-d)(2-d)\left\{1+\theta^{2}-2 \theta d /(1-d)\right\}} .
\end{gathered}
$$

Quando $k \rightarrow \infty$.

$$
\rho_{k}^{y} \sim \frac{(-d) !}{(d-1) !} a i^{2 d-1}
$$




\section{Capítulo 5}

\section{Estimação dos Parâmetros em Modelos ARFIMA(p,d,q)}

\subsection{Métodos de Estimação de $d$}

\subsubsection{Introdução}

Neste capítulo apresentamos os principais métodos de estimaçào do parâmetro de diferença fracionária, $d$, de modelos $A R F I M A(p, d, q)$. Na Seção 5.2 um algorítmo de estimação de todos os parâmetros é apresentado, pois a maioria dos métodos não estimam simultaneamente os parâmetros do modelo $A R F I M A(p, d, q)$. Os métodos de estimação se dividem em três grupos:

1. Método da funçào de autocorrelação de ordem 1, método da autocorrelação generalizado, método da auto correlação parcial e o método das ondaletas. Esses métodos foram elaborados para estimar o parâmetro $d$ somente em modelos $A R F I M A(0, d, 0)$.

2. Método de regressão utilizando o periodograma e o método de regressão utilizando o periodograma suavizado. Esses métodos foram elaborados para estimar $d$ em modelos $A R F I M A(p, d, q)$. mas não estimam os parâmetros simultaneamente.

3. Método de máxima verossimilhança. Este é o único método que estima simultanea- 
mente os parâmetros do modelo $A R F I M A(p, d . q)$.

Apesar dos métodos citados no primeiro grupo terem sido formulados para modelos ARF IM $A(0, d .0)$, eles podem ser aplicados ao modelo completo $A R F I M A(p, d, q)$ através de uma aplicação iterativa do algorítmo de estimação dado na Seção 5.2. Este algorítmo só não será utilizado no caso do método de máxima verossimilhança.

\subsubsection{Método da Função de Autocorrelação de Ordem 1}

Este método, proposto por Gupta \& Kumar (1991), é o método mais simples dentre os métodos que serão apresentados neste capítulo. Ele tem como base a relação entre $d$ e a função de autocorrelação de ordem 1, $\rho_{1}$, de um processo $\operatorname{ARFIMA}(0, \mathrm{~d}, 0)$. Tal relação é obtida através da função de autocorrelaçào, dada pelo Teorema 4.1.

$$
\rho_{k}=\frac{d(1+d) \ldots(k-1+d)}{(1-d)(2-d) \ldots(k-d)} \quad k=1,2, \ldots .
$$

que, para $k=1$, fornece

$$
d=\frac{\rho_{1}}{1+\rho_{1}}
$$

A proposta de Gupta \& Kumar foi de substituir $\rho_{1}$ pela autocorrelação amostral de ordem $1, \hat{\rho}_{1}$, ou seja, o estimador de $d$, neste caso, é dado por

$$
\hat{d}_{c}=\frac{\hat{\rho}_{1}}{1+\hat{\rho}_{1}} \text {. }
$$

Gupta \& Kumar (1991) mostram que, para $-\frac{1}{2}<d<0$, este é um estimador não viciado com distribuição assintótica normal, com variância dada por

$$
\frac{V}{T\left(1+\rho_{1}\right)^{4}}
$$

onde $T$ é o número de abservações da série temporal em questão e

$$
V=\sum_{k=1}^{\infty}\left(\rho_{k+1}+\rho_{k-1}-2 \rho_{1} \rho_{k}\right)^{2} .
$$

Assim,

$$
\sqrt{T}\left(\hat{d}_{c}-d\right) \sim A N\left(0, \frac{V}{\left(1+\rho_{1}\right)^{4}}\right) .
$$




\subsubsection{Método da Função de Autocorrelação Generalizado}

Seja $\left\{X_{t}, t=0,1, \ldots, T-1\right\}$ um processo $A R F I M A(0, d, 0)$. O método de estimação anterior utiliza apenas a informaçào que a função de autocorrelação de $X_{t}, \rho_{k}$, oferece para $k=1$, desconsiderando as informaçòes oferecidas para $k=2,3, \ldots, T-1$. Com o objetivo de incorporar essas "informaçòes" no método de estimaçào de d, Gupta \& Ji (1992) propuseram utilizar as autocorrelações amostrais $\hat{\rho}_{k}, k=1,2, \ldots, N$, onde $N$ é um número inteiro positivo tal que $N<T$. A construção deste estimador decorre da equação

$$
\rho_{k}=\rho_{k-1} \frac{k-1+d}{k-d}, \quad k=1,2, \ldots,
$$

que é obtida reescrevendo (5.1). Resolvendo essa equação em $d$, temos

$$
d=\frac{k \rho_{k}-(k-1) \rho_{k-1}}{\rho_{k}+\rho_{k-1}},
$$

que sugere um estimador preliminar de $d$ dado por

$$
\hat{d}(k)=\frac{k \hat{\rho}_{k}-(k-1) \hat{\rho}_{k-1}}{\hat{\rho}_{k}+\hat{\rho}_{k-1}}, k=1,2, \ldots, T,
$$

onde $\hat{\rho}_{k}$ é a função de autocorrelação amostral de ordem $k$. O estimador sugerido por Gupta $\&$ Ji é uma soma ponderada de $\hat{d}(k)$ para $k=1,2, \ldots, N$, dado por

$$
\hat{d}_{c g}=\sum_{i=1}^{N} a_{i} \hat{d}(i)
$$

onde $N<T$ e $\left\{a_{i}, i=1.2, \ldots, N\right\}$ é um conjunto de números reais não negativos (chamados de pesos) tais que $\sum_{i=1}^{T} a_{i}=1$.

A função peso sugerida por Gupta \& Ji é dada por

$$
a_{i}=\frac{\frac{1}{\operatorname{Var}(\dot{d}(i))}}{\sum_{j=1}^{N} \frac{1}{\operatorname{Var}(\dot{d}(i))}}, \quad i=1,2, \ldots, N,
$$

Nota: Na prática, $\operatorname{Var}(\hat{d}(i))$ é substituída pela variância amostral de $\hat{d}(i), \hat{\operatorname{Var}}(\hat{d}(i))$.

O teorema a seguir fornece a distribuição conjunta assintótica Normal de $\{\hat{d}(k)\}$, deduzida por Gupta \& Ji (1992). 
Notação:

$$
W_{i, j}= \begin{cases}\sum_{k=1}^{\infty}\left\{\left[\rho_{k+i}+\rho_{k-i}-2 \rho_{k} \rho_{i}\right]\left[\rho_{k+i}+\rho_{k-j}-2 \rho_{k} \rho_{j}\right]\right\} & \text { se } i, j \neq 0 \\ 0 & \text { caso contrário }\end{cases}
$$

Teorema 5.1 Seja $\left\{X_{t}\right\}$ um processo $A R F I M A(0 . d .0)$. definido por

$$
(1-B)^{d} X_{t}=Z_{t}
$$

onde $d<0$ e $Z_{t} \sim \operatorname{II} D\left(0 . \sigma^{2}\right)$.

Seja $\hat{D}=(\hat{d}(1)-d, \hat{d}(2)-d \ldots \hat{d}(m)-d)^{\prime}$. onde $m$ é qualquer inteiro positivo. Então,

$$
\sqrt{n} \hat{D} \sim A N\left(0 . V_{i}\right)
$$

onde

$$
\begin{gathered}
0=(0,0, \ldots 0)_{1 \times m} \quad \text { e } \quad V_{1}=\left[v_{i, j}\right]_{m \times m}, \\
v_{i, j}=\frac{(2 i-1)(2 j-1) u_{i, j}}{\left(\rho_{i}+\rho_{i-1}\right)^{2}\left(\rho_{j}+\rho_{j-1}\right)^{2}}
\end{gathered}
$$

sendo

$$
u_{i, j}=\left\{\rho_{i-1} \rho_{j-1} W_{i, j}-\rho_{i-1} \rho_{j} W_{i, j-1}-\rho_{i} \rho_{j-1} W_{i-1, j}+\rho_{1} \rho_{j} W_{i-1, j-1}\right\}
$$

Demonstração: Ver Gupta \& Ji (1992).

Teorema 5.2 Se $\left\{X_{t}\right\}$ for um processo $A R F I . M A(0 . d, 0)$ definido por $(1-B)^{d} X_{t}=Z_{t}$, onde $d<0$ e $Z_{t} \sim \operatorname{II} D\left(0, \sigma^{2}\right)$, entào

$$
\sqrt{n}\left(\hat{d}_{c g}-d\right) \sim A N\left(0 . V_{d}\right)
$$

onde $V_{d}=a^{\prime} V_{1} a$. Os termos $a=\left(a_{1}, a_{2}, \ldots a_{n}\right)$ e $V_{1}$ estào definidos em (5.8) e (5.9) respectivamente.

Demonstração: Ver Gupta \& Ji (1992). 


\subsubsection{Método da Função de Autocorrelação Parcial}

Este método foi proposto por Vinod (1991) e se baseia na funçào de autocorrelaçào parcial de um processo $A R F I M A(0, d, 0)$. dada pelo Teorema 4.1 .

$$
\phi_{k k}=\frac{d}{k-d} . \quad k=1.2 \ldots .
$$

Resolvendo a equaçào anterior em $d$, temos

$$
d=\frac{k \cdot o_{k \cdot k}}{1+o_{k \cdot k}} .
$$

Um possivel estimador de $d$ seria

$$
\hat{d}^{\times}(k)=\frac{\hat{i}_{k \cdot k}}{1+\hat{O}_{k k}} .
$$

$\hat{\phi}_{k k}$ é a autocorrelaçào parcial amostral de ordem $k$, em particular. para $k=1, \hat{\phi}_{k k}=\hat{\rho}_{1}$. Entretanto, ao escolher um único $k$ para obter o estimador de $d$, estamos perdendo as informaçòes sobre o parâmetro $d$ que a funçào de autocorrelação parcial oferece para os demais valores de $k$. Com o objetivo de nào perder tais informaçòes. Vinod (1991) propôs combinar todos os $\hat{d}^{\times}(k)$, para $k=1.2, \ldots T$, usando a funçào peso

$$
W_{k}= \begin{cases}\frac{1}{\operatorname{dp}(\hat{d}(k))} & \text { se } 0<\hat{d}(k)<\frac{1}{2} \\ 0 & \text { se } \hat{d}(k) \notin\left(0, \frac{1}{2}\right) .\end{cases}
$$

fornecendo o seguinte estimador para $d$

$$
\hat{d}_{c p}=\frac{\sum_{k=1}^{T} W_{k} \cdot \hat{d}^{\times}(k)}{\sum_{k=1}^{T} W_{k}^{*}} .
$$

\subsubsection{Método da Regressão Utilizando o Periodograma}

Este método foi proposto por Geweke \& Porter-Hudak (1983) e se baseia na equação que exibe a relaçào entre a funçào de densidade espectral de um processo $A R F I M A(p, d, q) \mathrm{e}$ a de um processo $A R M A(p, q)$. Tal equaçào foi reescrita para que se assemelhasse a uma 
equaçào de regressào simples. onde o coeficiente de inclinaçào é dado por $b=-d$. Para estimar $d$ foi proposto utilizar o método de regressào de mínimos quadrados. A seguir, será apresentado o processo de formaçào desse método.

Seja $\left\{X_{t}\right\}$ um processo $A R F I M A(p . d . q) \operatorname{com}|d|<\frac{1}{2}$. ou seja.

$$
X_{t}=(1-B)^{-d} \frac{\theta(B)}{O(B)} a_{t}
$$

ou

$$
X_{t}=\iota(B) a_{t}=\sum_{j=0}^{\infty} \iota_{j} a_{t-j}
$$

$\operatorname{com} \iota_{0}=1$ e $\iota \cdot(B)=(1-B)^{-\ell} \frac{\theta(B)}{\partial(B)}$. Esta forma de representaçào de $\left\{X_{t}\right\}$, denominada no Capítulo 3 de forma de choques aleatórios. representa o prossesso $\left\{X_{t}\right\}$ como uma saida de um filtro linear cuja entrada é dada pelo processo ruido branco $\left\{a_{t}\right\}$. Pelo Teorema 2.10 a funçào de densidacle espectral de $X_{t}$ é dada por

$$
f_{x}(\lambda)=|F(\lambda)|^{2} \quad f_{a}(\lambda)
$$

onde $f_{a}(\lambda)=\frac{\sigma_{a}^{2}}{2 \pi}$ é a funçào de densidade espectral de $a_{t} \mathrm{e}$

$$
F(\lambda)=\sum_{t=0}^{\infty} \psi_{t} \exp \{i \lambda t\}=\psi\left(\mathrm{e}^{i \lambda}\right)=\left(1-\mathrm{e}^{i \lambda}\right)^{-t l} \frac{\theta\left(\mathrm{e}^{i \lambda}\right)}{\partial\left(\mathrm{e}^{i \lambda}\right)} .
$$

De (5.16) temos que

$$
|F(\lambda)|^{2}=\left(2 \operatorname{sen} \frac{\lambda}{2}\right)^{-2 d} \frac{\left|\theta\left(\mathrm{e}^{i . \lambda}\right)\right|^{2}}{\left|O\left(\mathrm{e}^{i . \lambda}\right)\right|^{2}}
$$

assim, reescrevendo (5.15) temos

$$
f_{x}(\lambda)=\left(2 \operatorname{sen} \frac{\lambda}{2}\right)^{-2 t l} \frac{\sigma_{a}^{2}}{2 \pi} \frac{\left|\theta\left(\mathrm{e}^{i \lambda}\right)\right|^{2}}{\left|\delta\left(\mathrm{e}^{i \lambda}\right)\right|^{2}} .
$$

Por outro lado. $C_{t}=(1-B)^{d} X_{t}$ é um processo $A R . M A(p . q)$ e. por (3.18), $U_{t}$ tem função de densidade espectral dada por

$$
f_{u}(\lambda)=\frac{\sigma_{a}^{2}}{2 \pi} \frac{\left|\theta\left(\mathrm{e}^{i . \lambda}\right)\right|^{2}}{\left|\phi\left(\mathrm{e}^{i . \lambda}\right)\right|^{2}}
$$


Sendo assim. podemos reescrever (5.18). obtendo

$$
f_{x}(\lambda)=\left(2 \operatorname{sen} \frac{\lambda}{2}\right)^{-2 d} f_{u}(\lambda)
$$

Esta relaçào fatora a densidade espectral de $X_{i}$ em dois termos independentes, onde o termo $\left(2 \operatorname{sen} \frac{1}{2}\right)^{-2 d}$ descreve o comportamento de longa memória da série, enquanto $f_{u}(\lambda)$ descreve o comportamento de curta memória. Por outro lado, o comportamento de longa memória de uma série temporal. $X_{t}$. é revelado por sua funçào de densidade espectral, $f_{x}(\lambda)$, nas frequências próximas de zero. Com isso. é razoável pensar em estimar $d$ com base em estimativas de $f_{x}(\lambda)$ para $\lambda$ próximo de zero. Com esse objetivo. Geweke \& Porter-Hudak (198:3) utilizaram o periodograma de $X_{t} . I_{x}(\lambda)$. como estimador de $f_{x}(\lambda)$ e efetuaram algumas modificaçòes em (5.19). dadas a seguir.

Multiplicando ambos os lados de (5.19) por $f_{u}(0)$ e aplicando o logarítmo, obtemos

$$
\ln \left(f_{x}(\lambda)\right)=\ln \left(f_{u}(0)\right)-d \ln \left[\operatorname{tsen}^{2}\left(\frac{\lambda}{2}\right)\right]+\ln \left[\frac{f_{u}(\lambda)}{f_{u}(0)}\right] .
$$

Fazendo $\lambda=\lambda_{j, T}=2 \pi j / T$ e adicionando $\ln \left[I_{x}\left(\lambda_{j, T}\right)\right]$ a ambos os lados da equação anterior, temos

$$
\ln \left(I_{x}\left(\lambda_{j, T}\right)\right)=\ln \left(f_{u}(0)\right)-d \ln \left[4 \operatorname{sen}^{2}\left(\frac{\lambda_{j, T}}{2}\right)\right]+\ln \left[\frac{f_{u}\left(\lambda_{j, T}\right)}{f_{u}(0)}\right]+\ln \left[\frac{I_{x}\left(\lambda_{j, T}\right)}{f_{x}\left(\lambda_{j, T}\right)}\right] .
$$

O termo $\ln \left[\frac{f_{u}\left(\lambda_{1}, T\right)}{f_{u}(0)}\right]$ em $(5.21)$ pode ser desprezado quando se considera apenas as frequências $\lambda_{j, T}$ próximas de zero. Para isso, consideremos $j=1,2, \ldots n$ em (5.21), onde $n$ é um inteiro positivo, $n<T$, tal que quando $T \rightarrow \infty$ a frequência $\lambda_{j, T}$ se aproxima de zero e, consequentemente, o termo citado anteriormente também se aproxima de zero, podendo ser ignorado. Com isso, podemos escrever

$$
\ln \left(I_{x}\left(\lambda_{j, T}\right)\right) \approx \ln \left(f_{u}(0)\right)-d \ln \left[4 \operatorname{sen}^{2}\left(\frac{\lambda_{j, T}}{2}\right)\right]+\ln \left[\frac{I_{x}\left(\lambda_{j, T}\right)}{f_{x}\left(\lambda_{j, T}\right)}\right], j=1,2, \ldots, n
$$

O teorema enunciado a seguir assegura que, para $d<0$. os termos $\ln \left[\frac{I_{x}\left(\lambda_{,}, T\right)}{f_{x}\left(\lambda_{,}\right)}\right]$, $j=1,2 \ldots T-1$. sào assintoticamente independentes e identicamente distribuídos com média e variância finitas. 
Teorema 5.3 Seja $\left\{X_{t}\right\}$ um processo $A R F I . M A(p . d . q) \operatorname{com} d<0$ e $\left\{a_{t}\right\}$ um processo ruído branco. Quando $T \rightarrow \infty$ os termos $\left\{-\ln \left(\frac{I_{x}(\lambda, T)}{f_{x}(\lambda, T)}\right)\right\} . j=1.2 \ldots \ldots T-1$ seguem distribuições de Gumbel independentes com média 0.557216 (constante de Euler) e variância $\frac{\pi^{2}}{6}$.

Demonstraçĩo: Ver Reisen (1994).

Utilizando a notaçào

$$
\begin{array}{ll}
y_{j}=\ln \left(I_{x}\left(\lambda_{j, T}\right)\right) \quad & a=\ln \left(f_{u}(0)\right)-C . \\
x_{j}=\ln \left[4 \operatorname{sen}^{2}\left(\frac{\lambda_{j}, T}{2}\right)\right] \quad & b=-d . \\
\varepsilon_{j}=\ln \left[\frac{I_{f}\left(\lambda_{,}, T\right)}{f_{x}\left(\lambda_{j, T}\right)}\right]+C \quad \text { e } \quad C^{\prime}=E\left\{-\ln \left[\frac{I_{x}\left(\lambda_{,}\right)}{f_{x}\left(\lambda_{,}\right)}\right]\right\}=\text {constante de Euler. }
\end{array}
$$

temos

$$
y_{j}=a+b x_{j}+\varepsilon_{j}, \quad j=1,2 \ldots, n,
$$

que tem a forma de uma equação de regressào linear simples. Com isso, a idéia de Geweke \& Porter-Hudak (198:3) foi de aplicar o método de regressào de mínimos quadrados ordinários para estimar $d$. Mas. para isso, é necessário que os termos de $\left\{\varepsilon_{j}\right\}, j=1,2, \ldots, n$ sejam assintoticamente independentes e identicamente distribuidos com média zero e variância finita. Entretanto, isso não é válido, pois $n$ é fixo, ou seja, quando se faz $T \rightarrow \infty$ as frequências $\lambda_{j, T}$ permanecem fixas, impedindo que os teoremas de limite central usuais possam ser aplicados. Para solucionar esse problema. a idéia é fazer $n=g(T)$, tal que

$$
\lim _{T \rightarrow \infty} g(T)=\infty \quad \epsilon \quad \lim _{T \rightarrow \infty} \frac{g(T)}{T}=0 .
$$

Com isso, o teorema citado anteriormente pode ser aplicado aos termos $\left\{-\ln \left(\frac{I_{x}\left(\lambda_{3}, T\right)}{f_{x}\left(\lambda_{3}, T\right)}\right)\right\}$ de (5.23). Quanto a escolha adequada de $g(T)$. o mais indicado é escolher $g(T)=T^{\alpha}$ com $0<a<1$.

Agora, iremos considerar (5.23) para $j=1.2 \ldots g(T)$. Pelo Teorema 5.3, temos que os termos da sequência $\left\{\varepsilon_{j}\right\}=\left\{-\ln \left(\frac{I_{x}\left(\lambda_{,}, T\right)}{f_{x}\left(\lambda_{j}, T\right)}\right)\right\}-C$ são assintoticamente independentes e identicamente distribuídos conforme a distribuição de Gumbel de média 0 e variância $\pi^{2} / 6$. 
Sendo assim. o estimador de $d$. proposto por Gerreke $d$ Porter-Hudak. é dado por $\hat{d}_{p}=-\hat{b}$, onde

$$
\hat{b}=\frac{\sum_{i=1}^{g(T)}\left(x_{i}-\bar{x}\right) y_{i}}{\sum_{i=1}^{g(T)}\left(x_{i}-\bar{x}\right)^{2}}
$$

é o estimador do coeficiente de inclinaçào de (5.23) , b, obtido através do método de mínimos quadrados aplicado a uma regressào linear simples de $y_{1}, y_{2} \ldots, y_{g(T)}$ sobre $x_{1}, x_{2}, \ldots, x_{g(T)}$. A esperança e a variância desse estimador sào dadas por

$$
E\left(\hat{d}_{p}\right)=E(-\hat{b})=d \quad \text { e } \operatorname{Var}\left(\hat{d}_{p}\right)=\frac{\pi^{2}}{6 \sum_{i=1}^{g(T)}\left(x_{i}-\bar{l}\right)^{2}} .
$$

Teorema 5.4 (Gewele \& Porter-Hudak (198.3)). O estimador do parâmetro de longa memória. $\hat{d}_{p}$. tem distribuiçào assintótica normal dada por

$$
. N\left(d . \frac{\pi^{2}}{6 \sum_{i=1}^{g(T)}\left(x_{i}-\bar{x}\right)^{2}}\right) .
$$

$\operatorname{com} g(T)=c T^{\alpha}$, onde $0<\alpha<1$ e $c$ é uma constante qualquer.

Teorema 5.5 Suponha que $\left\{X_{t}\right\}$ seja um processo $A R F I M A(p, d . q)$, com $d<0$. Seja $I\left(\lambda_{j, T}\right)$ o periodograma de $\left\{X_{t}\right\}$ na frequência harmônica $\lambda_{j, T}=2 \pi j / T$ numa amostra de tamanho $T$. Seja $\hat{d}_{p}$ o estimador de mínimos quadrados de $d$ na equação de regressão obtida de $(5.22)$

$$
\ln \left\{I\left(\lambda_{j, T}\right)\right\}=\beta_{0}+d \ln \left\{4 \operatorname{sen}^{2}\left(\lambda_{j, T} / 2\right)\right\}+u_{j, T}, \quad j=1, \ldots, n .
$$

Então. existe uma função $g(T)$ com

$$
\lim _{T \rightarrow 0} g(T)=\infty \text { e } \lim _{T \rightarrow 0} g(T) / T=0,
$$

de tal forma que

(a) se $n=g(T)$, entào plim $\hat{d}_{p}=-d$;

(b) se $\lim _{T \rightarrow \infty}(\ln T)^{2} / g(T)=0$, entào

$$
\frac{\left(\hat{d}_{p}+d\right)}{\left\{\operatorname{vâr}\left(\hat{d}_{p}\right)\right\}^{1 / 2}} \stackrel{\mathcal{D}}{\rightarrow} N(0.1)
$$

onde vâr $\left(\hat{d}_{p}\right)$ é o estimador de mínimos quadrados de $\operatorname{var}\left(\hat{d}_{p}\right)$. 
Nota: Embora os resultados apresentados no Teoremas 5.3. 5.4 e 5.5 sejam válidos somente para $d<0$. Geweke $\&$ Porter-Hudak (198:3) mostram através de simulações que tais propriedades sào válidas para $d>0$.

\subsubsection{Método de Regressão Utilisando o Periodograma Suavizado}

Este método foi proposto por Reisen (1994). Com o objetivo de melhorar o método de regressão descrito na seção anterior, um estimador consistente e nào viciado de $f_{x}(\lambda)$ é utilizado no método de estimaçào no lugar do periodograma simples. Foi sugerido substituir o periodograma simples pelo periodograma suavizado em ( 5.21$)$, obtendo a seguinte equação

$$
\ln \left[f_{x}^{T}\left(\lambda_{j . T}\right)\right]=\ln \left[f_{u}(0)\right]-d \ln \left[4 \operatorname{sen}^{2}\left(\frac{\lambda_{j, T}}{2}\right)\right]+\ln \left[\frac{f_{u}\left(\lambda_{j . T}\right)}{f_{u}(0)}\right]+\ln \left[\frac{f_{. T}^{T}\left(\lambda_{j, T}\right)}{f_{x}^{\prime}\left(\lambda_{j . T}\right)}\right],
$$

onde $f_{x}^{T}\left(\lambda_{j, T}\right)$ é o periodograma suavizado da série $\left\{X_{t}\right\}$, dado pela Definição 2.11 , com $\lambda_{j, T}=2 \pi j / T, j=1.2, \ldots T$. que pode ser escrito como

$$
f_{x}^{T}\left(\lambda_{j, T}\right)=\frac{1}{2 \pi} \sum_{s=-m}^{m} k\left(\frac{s}{m}\right) R(s) \cos \left(s \lambda_{j, T}\right)
$$

onde $k(u)$ é uma funçào par e contínua no intervalo $(-1,1) \operatorname{com} h(0)=1$, chamada de função geradora da jancla espectral e $R(s)$ é a autocovariancia amostral de ordem $s$ de $\left\{X_{t}\right\}$. O parâmetro $m$ é uma funçào de $T$, tal que

$$
\lim _{T \rightarrow \infty} m=\infty \text { e } \lim _{T \rightarrow \infty} \frac{m}{T}=0 .
$$

Em geral, ecolhe-se $m=T^{\beta}, \quad 0<\beta<1$.

Como foi visto na seção anterior, o termo $\ln \left[\frac{f_{u}\left(\lambda_{1}, T\right)}{f_{u}(0)}\right]$ de $(5.25)$ pode ser desprezado se forem consideradas apenas as frequências $\lambda_{j . T}$ próximas de zero. Então, escolhendo $g(T)$ pelos mesmos critérios citados anteriormente. pode-se escrever, para $j=1,2, \ldots, g(T)$,

$$
\ln \left[f_{x}^{T}\left(\lambda_{j, T}\right)\right] \approx \ln \left[f_{u}(0)\right]-d \ln \left[4 \operatorname{sen}^{2}\left(\frac{\lambda_{j, T}}{2}\right)\right]+\ln \left[\frac{f_{x}^{T}\left(\lambda_{j, T}\right)}{f_{x}\left(\lambda_{j, T}\right)}\right]
$$

que tem a forma de uma equação de regressào linear simples do tipo

$$
y_{j}=a+b x_{j}+\varepsilon_{j} . \quad j=1,2 \ldots, g(T)
$$


onde

$$
\begin{aligned}
& y_{j}=\ln \left(f_{x}^{T}\left(\lambda_{j, T}\right)\right) \quad . \quad a=\ln \left(f_{u}(0)\right) \\
& x_{j}=\ln \left[4 \operatorname{sen}^{2}\left(\frac{\lambda, T}{2}\right)\right] . \quad b=-d \\
& \varepsilon_{j}=\ln \left[\frac{f_{x}^{T}\left(\lambda_{,}\right)}{f_{x}\left(\lambda_{j, T}\right)}\right] .
\end{aligned}
$$

A relação (5.28) sugere estimar $d$ através do método de mínimos quadrados. Entretanto, para isso é preciso assegurar que os termos de $\left\{\varepsilon_{j}\right\}$ sào nào correlacionados com média zero e variância constante. Por Priestley (1981). $f_{x}^{T}(\lambda)$ é assintoticamente não viciado com variância clada por

$$
\lim _{T \rightarrow \infty}\left[\operatorname{Var}\left(f_{x}^{T}(\lambda)\right)\right]= \begin{cases}f_{x}^{2}(\lambda) \frac{m}{T} \int_{-\infty}^{\infty} k^{2}(u) & \text { se } \lambda \neq 0 . \pm \pi \\ 2 f_{x}^{2}(\lambda) \frac{m}{T} \int_{-\infty}^{\infty} k^{2}(u) & \text { se } \lambda=0, \pm \pi\end{cases}
$$

Reisen (1994) mostrou que para qualquer funçào geradora de janela espectral, $k(u)$, que satisfaça as condições do Teorema 9.2.1 de Anderson (1971). o teorema abaixo é válido.

Teorema 5.6 Seja $f_{x}\left(\lambda_{j, T}\right)$ a funçào de densidade espectral de um modelo $A R F I M A(p, d, q)$, $d \in\left(-\frac{1}{2}, 0\right)$, e $f_{x}^{T}\left(\lambda_{j, T}\right)$ um estimador de $f_{x}\left(\lambda_{j, T}\right)$ como em (5.26). Então, os termos $\ln \left\{\frac{f_{x}^{T}(\lambda, T)}{f_{x}\left(\lambda_{,}, T\right)}\right\}$ são nào correlacionados com distribuiçào assintótica normal de média 0 e variância dado por

$$
\operatorname{Var}\left[\frac{f_{x}^{T}\left(\lambda_{j, T}\right)}{f_{x}\left(\lambda_{j, T}\right)}\right] \approx \begin{cases}\frac{. M}{T} \int_{-\infty}^{\infty} k^{2}(u) d u & \text { se } \lambda \neq 0, \pm \pi \\ \frac{2 . M I}{T} \int_{-\infty}^{\infty} k^{2}(u) d u & \text { se } \lambda=0, \pm \pi\end{cases}
$$

Do Teorema 5.6, quando $-1 / 2<d<0$, pode-se utilizar o estimador de mínimos quadrados de $b$ em (5.28) para estimar $d$. Deste modo. o estimador de $d$ é dado por $\hat{d}_{p s}=-\hat{b}$, onde

$$
\hat{b}=\frac{\sum_{i=1}^{g(T)}\left(x_{i}-\bar{x}\right) y_{i}}{\sum_{i=1}^{g(T)}\left(x_{i}-\bar{x}\right)^{2}}
$$

cuja variância. de acordo com Reisen (1994), é dada por

$$
\operatorname{Var}(\hat{l}) \approx \frac{M}{T \sum_{i=1}^{g(T)}\left(x_{i}-\bar{x}\right)^{2}} \int_{-\infty}^{\infty} k^{2}(u) d u \quad \text { se } \lambda \neq 0, \pm \pi
$$


Nota: Apesar do Teorema 5.6 ser válido somente para $-1 / 2<d<0$. Reisen (1994) mostra, através de simulaçòes. que estes resultados sào válidos quando $d>0$.

\subsubsection{Método de Máxima Verossimilhança}

O estudo do estimador de máxima verossimilhança (EMV) para observações dependentes é vasto e reúne muitos resultados. Sweeting (1980) apresentou as condições sob as quais o estimador de máxima verossimilhança é consistente e tem distribuição assintótica normal. Basawa \& Prakasa Rao (1983) e Basawa \& Scott (1983) apresentaram teoremas e exemplos nesta área. Nesse contexto. uma série temporal com comportamento de longa memória é classificada como sequência de forte dependencia e. portanto. o EMV para este tipo de série é um caso particular do problema citado anteriomente. Para determinar o EMV para sequências de forte dependência Fox (t. Taqqu (1986) aplicaram os resultados já obtidos para o caso de observaçòes dependentes e apresentaram as condiçòes que permitem que o estimador encontrado seja consistente e tenha distribuiçào assintótica normal. Tais condições são satisfeitas pelos processos $A R F I M A(p, d . q)$ e ruído Gaussiano fracionário, ambos definidos no Capítulo 4.

Definição 5.1 Seja $\left\{X_{j}, j=1,2, \ldots\right\}$ uma sequência Gaussiana estacionária com média $\mu$ e funçào de densidade espectral $\sigma^{2} f(\lambda, \theta),-\pi \leq \lambda \leq \pi$. onde $\mu, \sigma^{2}>0$ e o vetor $\theta \in E \subset \mathbb{R}^{p}$ sào parâmetros desconhecidos. A covariância de $X_{t}$ é dada por

$$
E\left(X_{j}-\mu\right)\left(X_{j+k}-\mu\right)=\sigma^{2} \gamma_{i k}(\theta)=\sigma^{2} \int_{-\pi}^{\pi} e^{i k \lambda} f(\lambda, \theta) d \lambda
$$

No caso de uma sequência de longa memória. $X_{j}$. a função de densidade espectral, $f(\lambda, \theta)$, apresenta o seguinte comportamento nas frequências próximas de zero

$$
f(\lambda, \theta) \sim|\lambda|^{-a\left(\theta_{)}\right.} G_{\theta}(\lambda) \text { quando } \lambda \rightarrow 0
$$

onde $0<\alpha(\theta)<1$ e $G_{\theta}(\lambda)$ varia suavemente quando $\lambda \rightarrow 0$.

$\mathrm{O}$ método de máxima verossimilança estima $\theta$ e $\sigma^{2}$ maximizando, com relação a $\theta$ e $\sigma^{2}$,

$$
f\left(X_{1}, \ldots, X_{T} ; \theta, \sigma^{2}\right)=\left(\frac{1}{\sqrt{2 \pi} \sigma}\right)^{T} \exp \left\{-\frac{Z^{\prime} R_{T}^{-1}(\theta) Z}{2 T \sigma^{2}}\right\}
$$


$\operatorname{com} Z=\left(X_{1}-\bar{X}_{T} \ldots X_{T}-\bar{X}_{T}\right)^{t}$. onde $\bar{X}_{T}=\frac{1}{T} \sum_{j=1}^{T} X_{j}$ e $R_{T}(\theta)$ é tal que $\sigma^{2} R_{T}(\theta)$ é a matriz de covariância de $X_{1} \ldots X_{T}$.

Como o cálculo da inversa de $R_{T}(\theta)$ é complicado computacionalmente, Fox \& Taqqu (1986) utilizam a proposta de Whittle (1951) que aproxima a inversa de $R_{T}(\theta)$ pela matriz de ordem $(T \times T), A_{T}(\theta)$. cuja entrada $(j, k)$ é dada por

$$
\left[\mathcal{A}_{T}(\theta)\right]_{j k}=a_{j-k}(\theta)=a_{s}(\theta)=\frac{1}{(2 \pi)^{2}} \int_{-\pi}^{\pi} e^{i s .}(f(\lambda, \theta))^{-1} d \lambda
$$

Desse modo. aplicar o método de máxima verossimilhança para estimar $\theta$ e $\sigma^{2}$ significa maximizar

$$
f\left(X_{1} \ldots X_{T}: \theta \cdot \sigma^{2}\right) \approx\left(\frac{1}{\sqrt{2 \pi} \sigma}\right)^{T} \exp \left\{-\frac{Z^{\prime} A_{T}(\theta) Z}{2 T \sigma^{2}}\right\}
$$

com relaçào a $\theta$ e $\sigma^{2}$. Assim, sào escolhidos os valores de $\theta$ e $\sigma^{2}$ que maximizam (5.32), denominados estimadores de máxima verossimilhança e denotados por $\hat{\theta}_{T}$ e $\hat{\sigma}_{T}^{2}$, respectivamente. Isto é equivalente a escolher $\hat{\theta}_{T}$ que minimize

$$
\sigma_{T}^{2}(\theta)=\frac{Z^{\prime} A_{T}(\theta) Z}{T}
$$

e depois fazer $\hat{\sigma}_{T}^{2}=\sigma_{T}^{2}\left(\hat{\theta}_{T}\right)$. O estimador de máximaverossimilhança de $d$ será denotado por $\hat{l}_{m v}$

A seguir serào dadas as condições sob as quais $\hat{\boldsymbol{\theta}}_{T}$ e $\hat{\sigma}_{T}^{2}$ são consistentes e têm distribuição assintótica normal.

Condições: Dizemos que $f(\lambda, \theta)$ satisfaz as condições $(1)-(6)$ se existir $0<\alpha(\theta)<1$ tal que para cada $\delta>0$

1. $g(\theta)=\int_{-\pi}^{\pi} \log f(\lambda . \theta) d \lambda$ pode ser diferenciada duas vezes sob o sinal da integral.

2. $f(\lambda, \theta)$ é contínua para todo $(\lambda . \theta) . x \neq 0, f^{-1}(\lambda, \theta)$ é contínua para todo $(\lambda, \theta)$, e

$$
f(\lambda, \theta)=O\left(|\lambda|^{-\alpha(\theta)-\delta}\right) \text { quando } \lambda \rightarrow 0 .
$$


3. $\frac{\partial}{\partial \theta_{3}} f^{-1}(\lambda . \theta)$ e $\frac{i^{2}}{i \theta_{k}, \theta_{j}} f^{-1}(\lambda . \theta)$ sào contínuas para todo $(\lambda . \theta)$.

$$
\begin{aligned}
& \frac{\partial}{\partial \theta_{j}} f^{-1}(\lambda . \theta)=O\left(|\lambda|^{\alpha(\theta)-j}\right) \quad \text { quando } \lambda \rightarrow 0 . \quad 1 \leq j \leq p, \quad \text { e } \\
& \frac{\partial^{2}}{\partial \theta_{k} \partial \theta_{j}} f^{-1}(\lambda . \theta)=O\left(|\lambda|^{\alpha(\theta)-\delta}\right) \text { quando } \lambda \rightarrow 0 . \quad 1 \leq j, k \leq p .
\end{aligned}
$$

4. $\frac{\partial}{\partial \lambda} f(\lambda, \theta)$ é contínua para todo $(\lambda, \theta), x \neq 0$. e

$$
\frac{\partial}{\partial \theta_{j}} f(\lambda, \theta)=O\left(|\lambda|^{\alpha(\theta)-\delta-1}\right) \text { quando } \lambda \rightarrow 0 \text {. }
$$

5. $\frac{\partial^{2}}{\partial \lambda \theta} f^{-1}(\lambda . \theta)$ é contínua para todo $(\lambda . \theta) . x \neq 0$. e

$$
\frac{\partial^{2}}{\partial \lambda \partial \theta_{j}} f^{-1}(\lambda . \theta)=O\left(|\lambda|^{\alpha(\theta)-j-1}\right) \text { quando } \lambda \rightarrow 0 . \quad 1 \leq j \leq p .
$$

6. $\frac{\partial^{3}}{\partial^{2} \lambda \theta_{j}} f^{-1}(\lambda . \theta) e ́$ contínua para todo $(\lambda . \theta) . r \neq 0$. e

$$
\frac{\partial^{3}}{\partial^{2} \lambda \dot{\partial} \theta_{j}} f^{-1}(\lambda, \theta)=O\left(|\lambda|^{\alpha\left(\theta_{)-\delta-2}\right)} \text { quando } \lambda \rightarrow 0, \quad 1 \leq j \leq p .\right.
$$

Os teoremas enunciados a seguir. apresentados por Fox \& Taqqu (1986), estabelecem sob que condiçòes os estimadores $\hat{\theta}_{T}$ e $\hat{\sigma}_{T}^{2}$ sào consistentes e têm distribuiçào assintótica normal.

Teorema 5.7 Se $f(\lambda, \theta)$ satisfaz as condições 2 e 4 , então com probabilidade 1

$$
\lim _{T \rightarrow \infty} \hat{\boldsymbol{\theta}}_{T}=\theta_{0} \quad \mathrm{e} \lim _{T \rightarrow \infty} \hat{\sigma}_{T}^{2}=\sigma_{0}^{2}
$$

onde $\theta_{0}$ e $\sigma_{0}^{2}$ sào os verdadeiros valores dos parâmetros $\theta$ e $\sigma^{2}$.

Teorema 5.8 Se as condiçòes $(1)-(6)$ sào satisfeitas então o vetor aleatório $\sqrt{T}\left(\hat{\boldsymbol{\theta}}_{T}-\boldsymbol{\theta}_{0}\right)$ converge em distribuiçào para um vetor aleatório normal com média $\mu$ e matriz de covariância $4 \pi W^{-1}\left(\theta_{0}\right)$, onde $W(\theta)$ é uma matriz $p \times p$ com entrada $(j, h)$ dada por

$$
w_{j k}(\theta)=\int_{-\pi}^{\pi} f(\lambda, \theta) \frac{\partial^{2}}{\partial \theta_{j} \partial \theta_{k}} f^{-1}(\lambda, \theta) d \lambda
$$

Teorema 5.9 Se $X_{j}-\mu$ é um processo ruído Gaussiano fracionário com $1 / 2<H<1$ ou um $A R F I M A(p, d, q) \operatorname{com} 0<d<1 / 2$ os resultados dos Teoremas 5.7 e 5.8 se aplicam e neste caso $\theta=\left\{o_{1}, \ldots o_{p}, \theta_{1}, \ldots, \theta_{q}\right.$ e $\left.d\right\}$

As demonstraçòes desses teoremas são encontradas em Fox \& Taqqu (1986). 


\subsubsection{Método de Ondaletas}

Considere o espaço $L^{2}(\mathbb{R})$ de funçòes mensuráveis. $f$. definidas na reta real $\mathbb{R}$, que satisfaz

$$
\int_{-\infty}^{\infty}|f(x)|^{2} d x<\infty
$$

Assim as funçòes $f(t)$ devem decair para zero quando $t \rightarrow \pm \infty$. O método de estimação estudado nesta seção se baseia em funçòes que geram $L^{2}(\mathbb{R})$ e que satisfazem (5.34). A idéia é considerar dilatações (compressões) e translaçòes de uma única função $\psi$ de modo a cobrir IR. Ou seja. consideramos a função

$$
\iota_{a, b}(x)=\frac{1}{\sqrt{|a|}} \cdot\left(\frac{x-b}{a}\right) \quad a . b \in \mathbb{Z} .
$$

onde $\frac{1}{\sqrt{|a|}}$ é um termo normalizador. ou seja. $\left\|\psi_{a, b}(x)\right\|=1$. Devido ao tipo de comportamento dessa funçào, ela é denominada de "wavelet" (ondaleta ou pequena onda). Como pode ser observado, a "wavelet" $\psi_{a, b}(x)$ é obtida atraves da translação e da dilatação de $\psi(x)$. Por isso, chamamos a de parâmetro de escala ou dilataçào e $b$ de parâmetro de translação. $\psi(x)$ é chamada de "wavelet mãe" ou "wavelet búsica" e obedece às seguintes condições:

- $\int_{-\infty}^{\infty} \psi(t) d t=0$, chamada de condição de admissibilidade.

- As funções $\psi_{a, b}, \quad a, b \in \mathbb{Z}$, sào ortonormais, para escalas de $a$ e $b$.

- $\int_{-\infty}^{\infty} t^{j} \psi(t) d t=0$ para $j=0,1.2, \ldots, M-1$, para algum $M \geq 1$ e

- $\int_{-\infty}^{\infty}\left|t^{M} \psi(t)\right| d t<\infty$

Usualmente tomamos valores especiais $a=2^{-j}$ e $b=2^{-j} k$. onde $j, k \in \mathbb{Z}$. Com isso, as ondaletas consideradas são dadas por

$$
\psi_{a, b}(x)=\psi_{2^{-\jmath}, k 2^{-\jmath}}(x)=\sqrt{2^{j}} \psi\left(\frac{x-2^{-j} k}{2^{-j}}\right)=\sqrt{2^{j}} \psi\left(2^{j} x-k\right)=\psi_{j, k}(x), \quad j, k \in \mathbb{Z} .
$$


Definição 5.2 Seja $X_{t}, t=0 . \pm 1 \ldots$ uma série temporal. Os coeficientes de ondaletas para $X_{t}$ sào dados pelo produto interno

$$
\left\langle X_{t} \cdot \psi_{j, k}(t)\right\rangle=\sqrt{2^{j}} \sum_{t=-\infty}^{\infty} X_{t}<\left(\frac{t-2^{-j} k}{2^{-j}}\right) .
$$

onde $j, k: \mathbb{Z}$.

Observação: Na prática, temos $X_{t}, t=0,1, \ldots, T$, uma série temporal finita, $\operatorname{com} T=2^{m}$, $m>0$ inteiro. Com isso, os coeficientes de ondaletas são dados pela transformada finita de ondaletas com respeito a $\psi$

$$
d_{j, k}^{(v)}=\sqrt{2^{j}} \sum_{t=0}^{T-1} X_{t} \cdot\left(\frac{t-2^{-j} k}{2^{-j}}\right) .
$$

com $j=0,1, \ldots, m-1$ e $k=0,1, \ldots, 2^{j}-1$. Desse modo. o valor do parâmetro $k$ depende de $j$, o que implica em dizer que fixado o valor de $j$ teremos um número correspondente de funções $\psi_{j, k}(t)$. Quanto menor o valor de $j$, menos funções $\psi_{j, k}(t)$ correspondentes. Por exemplo, quando $j=0$ temos apenas uma funçào $\psi_{0,0}(t)$ correspondente e se $j=2$ temos $2^{2}=4$ funçòes $\psi_{2,0}(t), \psi_{2,1}(t), \psi_{2,2}(t)$ e $\psi_{2,3}(t)$ correspondentes.

Na literatura encontramos vários tipos de ondaletas, onde a mais simples é a função de Haar, dada por:

$$
\psi_{j, k}^{(H)}(t)= \begin{cases}2^{j / 2}, & 2^{-j} k \leq t<2^{-j}(k+1 / 2) \\ 2^{j / 2}, & 2^{-j}(k+1 / 2) \leq t<2^{-j}(k+1) \\ 0, & \text { caso contrário }\end{cases}
$$

Outros exemplos de ondaletas são as "Daublets", "Symmlets" e "Coiflets" todas introduzidas por Daubechies. Essas funçòes sào de suporte compacto e não-simétricas. Para maiores detalhes, ver Daubechies (1992). As "Symmlets" sào utilizadas como "default" em alguns programas por serem as menos assimétricas. Neste trabalho, utilizamos o "software wavethresh" desenvolvido por Nason, que pode ser obtido livremente da biblioteca Statlib na ftp anônima (ftp lib.stat.cmu.edu/S/wavethresh). Este software opera dentro do pacote S-plus e existe apenas a versào para UNIX. Um roteiro para o uso do programa está em Nason \& Silvemann (1994). 
Teorema 5.10 Seja $X_{t}$ um processo $A R F I . M A(0 . d .0)$ de média zero. com $|d|<1 / 2$. A transformada finita de ondaletas. $d_{j . k}^{(v)}$. clada por (5.36), tem distribuiçào normal com média zero e variância aproximada $\sigma^{2}\left|2^{-j}\right|^{2 d}$. onde $\sigma^{2}$ é uma constante finita. Ver Jensen (1996) para maiores detalhes.

Se $X_{t}$ é um processo $A R F I M A(0, d, 0)$ com média zero e $|d|<1 / 2$, do Teorema 5.10 temos que

$$
R_{k}(j)=E\left[\left(d_{j, k}^{(4)}\right)^{2}\right]
$$

é a variância dos coeficientes de ondaletas e que $R_{k}(j) \cong \sigma^{2}\left|2^{-j}\right|^{2 d}$. Assim, aplicando o logarítmo obtemos a seguinte relaçào $\log$-linear entre $R_{k}(j)$ e d

$$
\ln R_{k}(j) \cong \ln \sigma^{2}+d \ln \left|2^{-i}\right|^{2}
$$

Por outro lado, podemos utilizar a variância amostral de $c_{j . k}^{(u \cdot)}$ para estimar $R_{k}(j)$, obtendo o estimador

$$
\hat{R}(j)=\frac{1}{2^{j}} \sum_{k=0}^{2^{\jmath}-1}\left(d_{j, k}^{(\psi)}\right)^{2},
$$

onde $j=0,1, \ldots, m-1$. Adicionando $\ln \hat{R}(j)$ em ambos os lados de (5.38) e fazendo $\varepsilon_{j}=\ln \hat{R}(j)-\ln R_{k}(j)$ chegamos na seguinte relaçào

$$
\ln \hat{R}(j) \cong \ln \sigma^{2}+d \ln \left|2^{-j}\right|+\varepsilon_{j}
$$

Diante da relaçào (5.40), Jensen (1996) sugere estimar o parâmetro de diferença fracionária, $d$, através do método de mínimos quadrados ordinários.

Pode-se demonstrar que $E(\hat{R}(j))=R_{k}(j)$ e $\operatorname{Var}(\hat{R}(j)) \rightarrow 0$, quando $j \rightarrow \infty$. Pela lei dos grandes números de Markov, $\hat{R}(j)$ converge em probabilidade para $R_{k}(j)$ quando $j \rightarrow \infty$. Sendo assim, podemos escrever que

$$
\ln \hat{R}(j)=\ln R_{k}(j)+o_{p}(1)
$$

Substituindo $R_{k}(j)$ por $\sigma^{2}\left|2^{-j}\right|^{2 d}$. temos

$$
\ln \hat{R}(j)=\ln \sigma^{2}+d \ln \left|2^{-j}\right|^{2}+o_{p}(1) .
$$


Ou seja. quando $j \rightarrow x$. o estimador de mínimos quadrados ordinários. $\hat{d}_{w}$. obtido através da relaçào (5.41). oferece uma estimativa do parâmetro de diferença fracionária, $d$, do processo ARFIMAA(0.d.0).

Nota: Como foi visto anteriormente, o número de funçòes $\iota_{j, k}(t)$. para $j$ fixado, varia de acordo com os valores de $j$. Logo, o número de coeficientes de ondaletas, $d_{j, k}^{(\psi)}$, para a escala $j$ também varia. Na prática. nào utilizamos os estimadores $\hat{R}(j)$ para $j$ "pequeno". Jensen (1996), através de simulaçòes, sugere que se utilize $\hat{R}(j)$ para $j=2.3, \ldots, m-1$ no método de estimação de $d$. Com isso, o estimador do parâmetro $d$ é obtido utilizando o método de mínimos quadrados na regressào linear simples:

$$
\ln \hat{R}(j)=\ln \sigma^{2}+d \ln \left|2^{-j}\right|^{2}+o_{p}(1) . \quad j=2.3 \ldots . m-1 .
$$

onde $m$ é tal que $T=2^{m}$.

\subsection{Algorítmo para ajustamento de um modelo ARFI- $\operatorname{MA}(p, d, q)$}

Esta seção apresenta um algorítmo para se estimar os parâmetros autoregressivos e de médias móveis no modelo ARFIMIA(p,d,q). Na seçào anterior vimos os métodos de estimação do parâmetro $d$, que em geral é estimado separadamente dos outros parâmetros do modelo, exceçào feita para o estimador de máxima verossimilhança. Devido a esse fato, apresentamos um método que utiliza um filtro que retira da série o componente de longa memória, referente ao parâmetro $d$. Desse modo, conseguimos estimar os demais parâmetros através dos métodos tradicionais.

Temos o estimador de máxima verossimilhança que estima todos os parâmetros simultaneamente e, por isso, nào necessita do algorítmo apresentado nesta seção para estimar os parâmetros. Os estimadores do periodograma e do periodograma suavizado foram elaborados para modelos $A R F I . M A(p, d . q)$, mas nào estimam os parâmetros simultaneamente. Já os demais estimadores têm seus métodos baseados em modelos $A R F I M A(0, d, 0)$ o que implica num método recursivo para estimar os parâmetros. Portanto, temos três classes de estimadores de $d$ e para cada classe o método de estimação dos demais parâmetros é 
diferente.

Como foi dito anteriormente. após estimar o parâmetro d no modelo $A R F I M A(p, d, q)$ precisamos filtrar a série de tal forma a eliminar o componente de longa memória. Para isso, vamos nos basear em (5.19)

$$
f_{x}(\lambda)=\left(2 \operatorname{sen} \frac{\lambda}{2}\right)^{-2 d} f_{u}(\lambda)
$$

onde $f_{x}(\lambda)$ é a densidade espectral do processo $X_{t} \sim A R F I M A(p, d, q)$ e $f_{u}(\lambda)$ é a função de densidade espectral de um processo $I_{t} \sim A R M . A(p, q)$. De (5.43) obtemos

$$
f_{u}(\lambda)=\left(2 \operatorname{sen} \frac{\lambda}{2}\right)^{2 t} f_{x}(\lambda)
$$

e é esta relação que iremos utilizar para eliminar a componente de longa memória da série. A seguir apresentamos um algorítmo para estimar os parâmet ros do modelo $A R F I M A(p, d, q)$. Considere $\left\{X_{t}, t=0,1, \ldots, T-1\right\}$.

Passo 1: Obter uma estimativa de $d, \hat{d}$.

Passo 2: Obter a transformada discreta de Fourier de $X_{t}, I_{x}\left(\lambda_{j, T}\right)$. nas frequências harmônicas $\lambda_{j, T}=2 \pi j / T . j=1,2, \ldots, T$.

Passo 3: Com base em (5.44), calcular

$$
I_{u}\left(\lambda_{j, T}\right)=\left(2 \operatorname{sen} \frac{\lambda_{j, T}}{2}\right)^{2 \hat{d}} I_{x}\left(\lambda_{j, T}\right)
$$

que é a estimativa da transformada discreta de Fourier do processo $U_{t} \sim A R M A(p, q)$.

Passo 4: Aplicar a transformada inversa de Fourrier em $T_{u}\left(\lambda_{j, T}\right)$ e obter $\hat{U}_{t}$.

Passo 5: Obter as estimativas dos parâmetros autoregressivos $\left(\hat{o}_{1}, \hat{\phi}_{2}, \ldots, \hat{\phi}_{p}\right)$ e de médias móveis $\left(\hat{\theta}_{1}, \hat{\theta}_{2}, \ldots, \hat{\theta}_{q}\right)$ de $\hat{U}_{t}$ através dos métodos usuais de estimaçào, por exemplo, o método de máxima verossimilhança.

Passo 6: C'alcular

$$
\frac{\hat{\phi}(B)}{\hat{\theta}(B)} \cdot X_{t}=Z_{t} .
$$


onde $\hat{o}(B)=1-\hat{o}_{1} B-\ldots \ldots-\hat{o}_{p} B^{p}$ e $\hat{\theta}(B)=1-\hat{\theta}_{1} B . \ldots-\hat{\theta}_{1} B^{q}$. Assim. $Z_{t}$ pode ser considerado um processo ARFI.MA(0.d.0).

Passo 7: Utilizar $Z_{t}$ para estimar $d$, obtendo $\hat{d}$.

Passo 8: Voltar ao Passo 3 até que as estimativas convirjam.

Se forem utilizados os estimadores do periodograma e do periodograma suavizado para estimar $d$, o método de estimação dos parâmetros se resume aos j) primeiros passos. Caso contrário, o método é composto desses oito passos, exceto o estimador de máxima verossimilhança que estima simultaneamente todos os parâmetros do modelo $A R F I M A(p, d, q)$. 


\section{Capítulo 6}

\section{Simulações}

Neste capítulo comparamos os estimadores apresentados no C'apítulo $5 . \hat{d}_{c} \cdot \hat{d}_{c g}, \hat{d}_{p}, \hat{d}_{p s}, \hat{d}_{m v}$ e $\hat{d}_{w}$, com base em simulaçòes de amostras de processos ARF I.M.A(p.d.q). Foram simuladas amostras para modelos $A R F I M A(0, d, 0), A R F I M A(1, d, 0)$ e $A R F I M A(0, d, 1)$. No caso do modelo $A R F I M A(0, d, 0)$, foram simuladas amostras para os seguintes valores de $d: 0,1$; 0,$2 ; 0,3 ; 0,4$ e 0,45 , resultando em cinco modelos. No caso dos modelos ARF IMA $(1, d, 0) \mathrm{e}$ $A R F I M A(0, d, 1), d$ assume apenas um valor: 0,3 e os valores do parâmet ro auto-regressivo e de médias móveis são 0,7 e $-0,6$, respectivamente. Para cada modelo citado acima foram feitas 1.000 simulações de amostras de tamanho 128 . 256 e 512 . Abaixo, temos uma tabela que resume as simulações feitas, onde $\phi_{1}$ é o parâmetro auto-regressivo de ordem 1 e $\theta_{1}$ é o parâmetro de médias móveis de ordem 1.

\begin{tabular}{|l|c|c|c|}
\hline \multirow{2}{*}{ Características } & \multicolumn{3}{|c|}{ Modelos } \\
\cline { 2 - 4 } & ARFIMA $(0, d .0)$ & ARFI.MA(1.d.0) & ARFIMA $(0, d, 1)$ \\
\hline Valores de $d$ & $0.1,0,2,0,3,0,4,0,45$ & 0.3 & 0,3 \\
Valor de $\phi_{1}$ & - & 0.7 & - \\
Valor de $\theta_{1}$ & - & - & $-0,6$ \\
Tamanhos de amostra & $128,256,512$ & 128.256 .512 & $128,256,512$ \\
Número de simulaçòes & 1.000 & 1.000 & 1.000 \\
\hline
\end{tabular}


Fazendo um breve resumo dos estimadores de $d$. temos:

1. Estimador da autocorrelaçào de ordem 1

$$
\hat{d}_{c}=\frac{\hat{\rho}_{1}}{1+\hat{\rho}_{1}}
$$

onde $\hat{\rho}_{1}$ é a autocorrelaçào amostral de ordem um de um processo $\operatorname{ARFIMA}(0, \mathrm{~d}, 0)$.

2. Estimador da autocorrelação generalizado

$$
\hat{d}_{i g}=\sum_{i=1}^{N} a_{i} \hat{d}(i)
$$

onde os pesos $a_{i}$ sào dados em 5.6 e $\hat{d}(k)=\frac{k \hat{p}_{k}-(k-1) \hat{p}_{k-1}}{\hat{p}_{k}+\hat{p}_{k-1}}$. onde $\hat{\rho}_{k}$ é a autocorrelaçào amostral de ordem k de um processo ARFIM.A(0.d.0). Escolhemos $N=30$, com base nos resultados das simulaçòes feitas por Gupta (t Ji (1992).

3. O estimador do periodograma $\hat{d}_{p}$ é obtido através do método de regressão mínimos quadrados aplicado à equaçào

$$
\ln \left(I_{x}\left(\lambda_{j, T}\right)\right)=a-d \ln \left[4 \operatorname{sen}^{2}\left(\frac{\lambda_{j, T}}{2}\right)\right]+\Sigma_{j}, j=1,2, \ldots, g(T)
$$

onde $I_{x}\left(\lambda_{j, T}\right)$ é o periodograma de um processo ARFIMA(p.d.q), a função $g(T)=T^{\alpha}$, onde $\alpha=0,5$ e $T$ é o tamanho da amostra.

4. O estimador do periodograma suavizado. $\hat{d}_{p s}$, é obtido através do método de regressão de mínimos quadrados aplicado à equaçào

$$
\ln \left(f_{x}^{T}\left(\lambda_{j . T}\right)\right)=a+-d \ln \left[4 \operatorname{sen}^{2}\left(\frac{\lambda_{j . T}}{2}\right)\right]+\varepsilon_{j}, \quad j=1,2, \ldots, g(T)
$$

onde $f_{x}^{T}\left(\lambda_{j, T}\right)$ é o periodograma suavisado de um processo $\operatorname{ARFIMA}(\mathrm{p}, \mathrm{d}, \mathrm{q})$, a função $g(T)=T^{\alpha}$. onde $\alpha=0.5$ e $T$ é o tamanho da amostra. Utilizamos a janela espectral de Parzen para obter o periodograma suavizado $f_{x}{ }^{T}\left(\lambda_{j, T}\right)$. Quanto ao ponto de truncamento da janela espectral. escolhemos $m=T^{3}$. $\operatorname{com} \beta=0,9$. Tal decisão foi 
tomada com base no estudo de Reisen (1994). que após simulaçōes sugere esse ponto de truncamento. A janela de Parzen é dacla por

$$
k(u)= \begin{cases}1-6 u^{2}+6|u|^{3} . & |u| \leq \frac{1}{2} \\ 2(1-|u|)^{3}, & \frac{1}{2} \leq u \leq 1 \\ 0, & |u|>1 .\end{cases}
$$

5. O estimador das ondaletas, $\hat{d}_{w}$, é obtido através do método de regressão de mínimos quadrados aplicado à equaçào

$$
\ln \hat{R}(a)=\ln \sigma^{2}+d \ln \left|2^{-a}\right|^{2}+o_{p}(1), \quad a=2,3 \ldots, \log _{2}(T) .
$$

onde $\hat{R}(a)$ é o estimador da variância dos coeficientes de ondaletas, $d_{j, k}^{(\psi)}$.

Para obter os coeficientes das ondaletas. $d_{j, k}^{(\psi)}$, utilizamos a transformada discreta de ondaletas. em um pacote de programas para a liguagem S-Plus. elaborados por Nason (1994), denominado "wavethresh", que está disponível na Internet. ver Nason (1994). Esse pacote utiliza duas famílias de ondaletas ortonormais com suporte compacto, cujos nomes são: DaubExPhase e DaubLsymm, definidas em Daubechies (1992). Em nossos estudos utilizamos a família de ondaletas DaubExPhase $(\mathrm{N})$, onde $N$ é o número do filtro da ondaleta, quanto maior for o $N$ mais suave será a ondaleta. Foram feitas simula c oes para vários valores de $\mathrm{N}$ e foi escolhido para a apresentaçào dos resultados o valor $\mathrm{N}=2$.

O programa utilizado para obter as simulaçòes foi o S-Plus, que possui uma rotina própria para simular modelos $A R F I M A(p, d, q)$. O estimador de máxima verossimilhança foi obtido diretamente, através de uma rotina que o calcula para modelos $A R F I M A(p, d, q)$. Quanto aos demais estimadores, foram feitos programas especiais, também para o S-Plus, que estão no Apêndice A. Nas seções posteriores mostraremos os resultados obtidos com as simulações para cada um dos modelos citados anteriormente e. com base nesses resultados, compararemos os diversos estimadores de $d$.

\subsection{Resultados para os modelos $\operatorname{ARFIMA}(0, \mathrm{~d}, 0)$}

O modelo $A R F I M A(0, d, 0)$ é o mais simples dos modelos de longa memória. Ao simular amostras de cinco modelos $A R F I M A(0 . d, 0)$ diferentes. onde $d=0,1,0,2,0,3,0,4$ e 0,45 , 
temos como objetivo comparar os estimadores de $d$ para diferentes valores de $d$. Além disso, como sabemos que um processo $A R F I M A(p . d . q)$ é estácionario se $d<0,5$, queremos avaliar o quanto os estimadores de $d$ pioram quando este se aproxima do limite de não estacionariedade. Foram simuladas amostras de três tamanhos diferentes, $T=128,256$ e 512 , com o objetivo de avaliar as propriedades assintóticas dos estimadores para esses diferentes valores de $d$.

As propriedades dos estimadores são avaliadas através de:

(a) variância estimada de $\hat{d}$

$$
\hat{\operatorname{Var}}\left(\hat{d}_{j}\right)=\frac{\sum_{i=1}^{1000}\left(\hat{d}_{i j}-\bar{d}_{j}\right)^{2}}{999}
$$

onde $j$ se refere ao método utilizado para obtençào do estimador de $d$ e $\bar{d}_{j}=\frac{\sum_{i=1}^{1000} \dot{d}_{\imath j}}{1000}$,

(b) viés estimado de $\hat{d}$

$$
\hat{B}\left(\hat{d}_{j}\right)=\frac{\sum_{i=1}^{1000}\left(\hat{d}_{i j}-d\right)}{1000} \mathrm{e}
$$

(c) erro quadrático médio estimado

$$
E \hat{Q} M\left(\hat{d}_{j}\right)=\hat{\operatorname{Var}}\left(\hat{d}_{j}\right)+\left(\hat{B}\left(\hat{d}_{j}\right)\right)^{2} .
$$

A Tabela 6.1 sintetiza os resultados obtidos com as simulaçòes. Apresentamos as estimativas da variância e do viés de todos os estimadores de $d$ nos cinco modelos $\operatorname{ARF} \operatorname{IM} A(0, d, 0)$, $d=0,1,0,2,0,3,0,4$ e 0,45 . A tabela se refere aos resultados obtidos para amostras de tamanho $T=128.256$ e 512 . Através dessa tabela podemos analisar os estimadores segundo o viés e a variância estimados, obtidos nas simulaçòes para os diferentes casos apresentados. Com base nos resultados, podemos afirmar que:

1. Para tamanhos de amostra 128 e 256 , as estimativas das variâncias dos estimadores $\hat{d}_{c}$ e $\hat{d}_{c g}$ tendem a diminuir quando o valor do parâmetro $d$ aumenta. Entretanto, para $T=512$, esse comportamento nào se verifica, indicando que não há relação específica da variância com os valores do parâmetro $d$. 
2. As estimativas das variâncias dos demais estimadores nào apresentam tendência de crescimento ou de decrescimento em relaçào aos valores de $d$ para todos os tamanhos de amostra utilizados. Isso indica que, no caso de modelos $A R F I M A(0, d, 0)$, a variância desses estimadores não depende do valor que $d$ assume.

3. Os estimadores $\hat{d}_{c}, \hat{d}_{c g}, \hat{d}_{m v}$ e $\hat{d}_{w}$ apresentam estimativas do viés que aumentam em valor absoluto quando o parâmetro $d$ se aproxima do limite de não estacionariedade, 0,5 . Para os dois primeiros estimadores esse crescimento do viés é bem mais acentuado que para $\hat{d}_{m v}$ e $\hat{d}_{u}$. Esse comportamento acontece para todos os tamanhos de amostra utilizados.

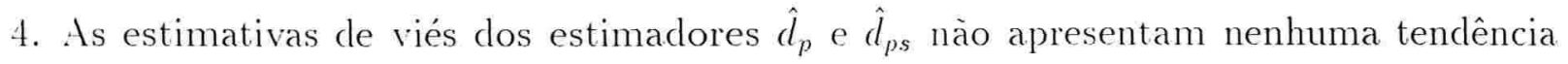
com relaçào aos valores que o parâmetro $d$ assume.

5. Todos os estimadores apresentam uma diminuição na variância e no viés estimado quando aumentamos o tamanho da amostra nas simulaçòes. Tal fato confirma as propriedades assintóticas dos estimadores.

Tabela 6.1 - Estimativas do viés e da variância do parâmetro $d$ de um modelo ARFIMA(0,d,0)

\begin{tabular}{|c|c|c|c|c|c|c|c|c|c|c|}
\hline \multirow[t]{2}{*}{$\mathrm{T}=128$} & \multicolumn{2}{|c|}{$\mathrm{d}=0,1$} & \multicolumn{2}{|c|}{$\mathrm{d}=0,2$} & \multicolumn{2}{|c|}{$\mathrm{d}=0.3$} & \multicolumn{2}{|c|}{$\mathrm{d}=0.4$} & \multicolumn{2}{|c|}{$\mathrm{d}=0,45$} \\
\hline & $\operatorname{Var}(\hat{d})$ & $\hat{B}(\hat{d})$ & $\operatorname{Var}(\hat{d})$ & $\hat{B}(\hat{d})$ & $\operatorname{Var}(\hat{d})$ & $\hat{B}(\hat{d})$ & $\operatorname{Var}(\hat{d})$ & $\hat{B}(\hat{d})$ & $\operatorname{Var}(\hat{d})$ & $\hat{B}(\hat{d})$ \\
\hline$\hat{d}_{c}$ & 0,006 & $-0.02+$ & 0,004 & $-0,037$ & 0,004 & -0.058 & 0,002 & $-0,088$ & 0,002 & $-0,108$ \\
\hline$\hat{d}_{c g}$ & 0,006 & $-0,021$ & 0.005 & $-0,034$ & 0,004 & 0.056 & 0,003 & $-0,091$ & 0,002 & $-0,113$ \\
\hline$\hat{d}_{p}$ & 0,061 & $-0,003$ & 0.065 & 0,015 & 0,063 & 0.012 & 0.062 & 0,000 & 0,063 & 0,033 \\
\hline$\hat{d}_{p s}$ & 0,037 & $-0,063$ & 0.033 & $-0,066$ & $0,0+1$ & $-0.0 \bar{\tau} 1$ & $0.0+2$ & $-0,060$ & 0,040 & $-0,067$ \\
\hline$\hat{d}_{m v}$ & 0,004 & $-0,02 \bar{\imath}$ & 0.006 & $-0,035$ & 0,005 & $-0.0+2$ & 0,004 & $-0,052$ & 0,004 & $-0,064$ \\
\hline$\hat{d}_{w}$ & 0.017 & -0.053 & 0.016 & $-0,063$ & 0,017 & $-0.0 \neg 1$ & 0,016 & $-0,073$ & 0,018 & $-0,084$ \\
\hline
\end{tabular}




\begin{tabular}{|c|c|c|c|c|c|c|c|c|c|c|}
\hline \multirow[t]{2}{*}{$\mathrm{T}=256$} & \multicolumn{2}{|c|}{$\mathrm{d}=0.1$} & \multicolumn{2}{|c|}{$\mathrm{d}=0.2$} & \multicolumn{2}{|c|}{$d=0.3$} & \multicolumn{2}{|c|}{$\mathrm{d}=0.4$} & \multicolumn{2}{|c|}{$\mathrm{d}=0,45$} \\
\hline & $\operatorname{Var}(\hat{d})$ & $\hat{B}(\hat{d})$ & $\operatorname{Var}(\hat{d})$ & $\hat{B}(\hat{l})$ & $\operatorname{Var}(\hat{d})$ & $\hat{B}(\hat{d})$ & $\operatorname{Var}(\hat{d})$ & $\hat{B}(\hat{d})$ & $\operatorname{Var}(\hat{d})$ & $\hat{B}(\hat{d})$ \\
\hline$\hat{d}_{c}$ & 0,003 & $-0,014$ & 0,002 & $-0,023$ & 0,002 & $-0,039$ & 0.001 & $-0,066$ & 0,001 & $-0,08.5$ \\
\hline$\hat{d}_{c g}$ & 0,003 & -0.012 & 0.002 & $-0,020$ & 0.002 & $-0,038$ & 0,001 & $-0,069$ & 0,001 & $-0,091$ \\
\hline$\hat{d}_{p}$ & 0,041 & $-0,000$ & 0.047 & $-0,006$ & 0,040 & 0,009 & 0.042 & 0,007 & 0,044 & 0,020 \\
\hline$\hat{d}_{p s}$ & 0,025 & $-0,044$ & 0,025 & $-0,0.50$ & $0,02 \tau$ & -0.053 & 0,029 & $-0,041$ & 0,028 & $-0,036$ \\
\hline$\hat{d}_{m v}$ & 0,002 & $-0,020$ & 0,003 & $-0,020$ & 0,003 & $-0,021$ & 0,002 & $-0,028$ & 0,002 & $-0,038$ \\
\hline$\hat{d}_{w}$ & 0,010 & -0.047 & 0,010 & $-0,052$ & 0,010 & $-0,061$ & 0.010 & $-0,059$ & 0,010 & $-0,062$ \\
\hline \multirow[t]{2}{*}{$\mathrm{T}=512$} & \multicolumn{2}{|c|}{$\mathrm{d}=0,1$} & \multicolumn{2}{|c|}{$\mathrm{d}=0,2$} & \multicolumn{2}{|c|}{$\mathrm{d}=0.3$} & \multicolumn{2}{|c|}{$\mathrm{d}=0,4$} & \multicolumn{2}{|c|}{$\mathrm{d}=0,4.5$} \\
\hline & $\operatorname{Var}(\hat{d})$ & $\hat{B}(\hat{d})$ & $\operatorname{Var}(\hat{d})$ & $\hat{B}(\hat{d})$ & $\operatorname{Var}(\hat{d})$ & $\hat{B}(\hat{d})$ & $\operatorname{Var}(\hat{d})$ & $\hat{B}(\hat{d})$ & $\operatorname{Var}(\hat{d})$ & $\hat{B}(\hat{d})$ \\
\hline$\hat{d}_{c}$ & 0.002 & -0.006 & 0,001 & $-0,013$ & 0,001 & -0.026 & 0,001 & $-0,052$ & 0,001 & $-0,070$ \\
\hline$\hat{d}_{c g}$ & 0.002 & -0.005 & 0.001 & -0.011 & 0.001 & -0.026 & 0.001 & -0.056 & 0,001 & $-0,078$ \\
\hline$\hat{d}_{p}$ & 0,026 & 0.013 & 0.025 & $-0,000$ & 0.026 & 0.008 & $0.02 \bar{\imath}$ & 0,011 & 0,029 & 0,008 \\
\hline$\hat{d}_{p s}$ & 0,016 & $-0,039$ & 0,018 & $-0,036$ & 0,017 & $-0,02 \bar{\imath}$ & 0,023 & $-0,032$ & 0,023 & $-0,039$ \\
\hline$\hat{d}_{m v}$ & 0,001 & $-0,010$ & 0.001 & $-0,011$ & 0.001 & $-0,013$ & 0,001 & $-0,014$ & 0,001 & $-0,021$ \\
\hline$\hat{d}_{w}$ & 0,006 & $-0,035$ & 0.006 & $-0,037$ & $0,00 T$ & $-0,043$ & $0,00 \bar{\imath}$ & $-0,044$ & 0,007 & $-0,045$ \\
\hline
\end{tabular}

Para melhor analisar os estimadores apresentamos alguns gráficos que comparam os estimadores em relação à variância e ao viés. A Figura 6.1 apresenta as variâncias estimadas dos estimadores de $d$ obtidas nas simulaçòes de amostras de tamanho $\mathrm{T}=128,256$ e 512 , respectivamente, dos modelos $A R F I M A(0, d, 0), d=0,1,0,2,0,3,0,4$ e 0,45 . Analizando esses gráficos, podemos perceber que:

1. Os estimadores $\hat{d}_{c}, \hat{d}_{c g}$ e $\hat{d}_{m v}$ são muito semelhantes com relação à variância, além de apresentarem as menores estimativas das variâncias para todos os valores que o parâmetro $d$ assume.

2. O estimador $\hat{d}_{w}$ aparece com a segunda menor variância para todos os valores de $d$.

3. Os estimadores que se baseiam no periodograma, $\hat{d}_{p}$ e $\hat{d}_{p s}$, são os que apresentam a maior variância.

4. A classificação dos estimadores com relaçào à variância permanece a mesma para todos os tamanhos de amostra utilizados nas simulaçòes. 

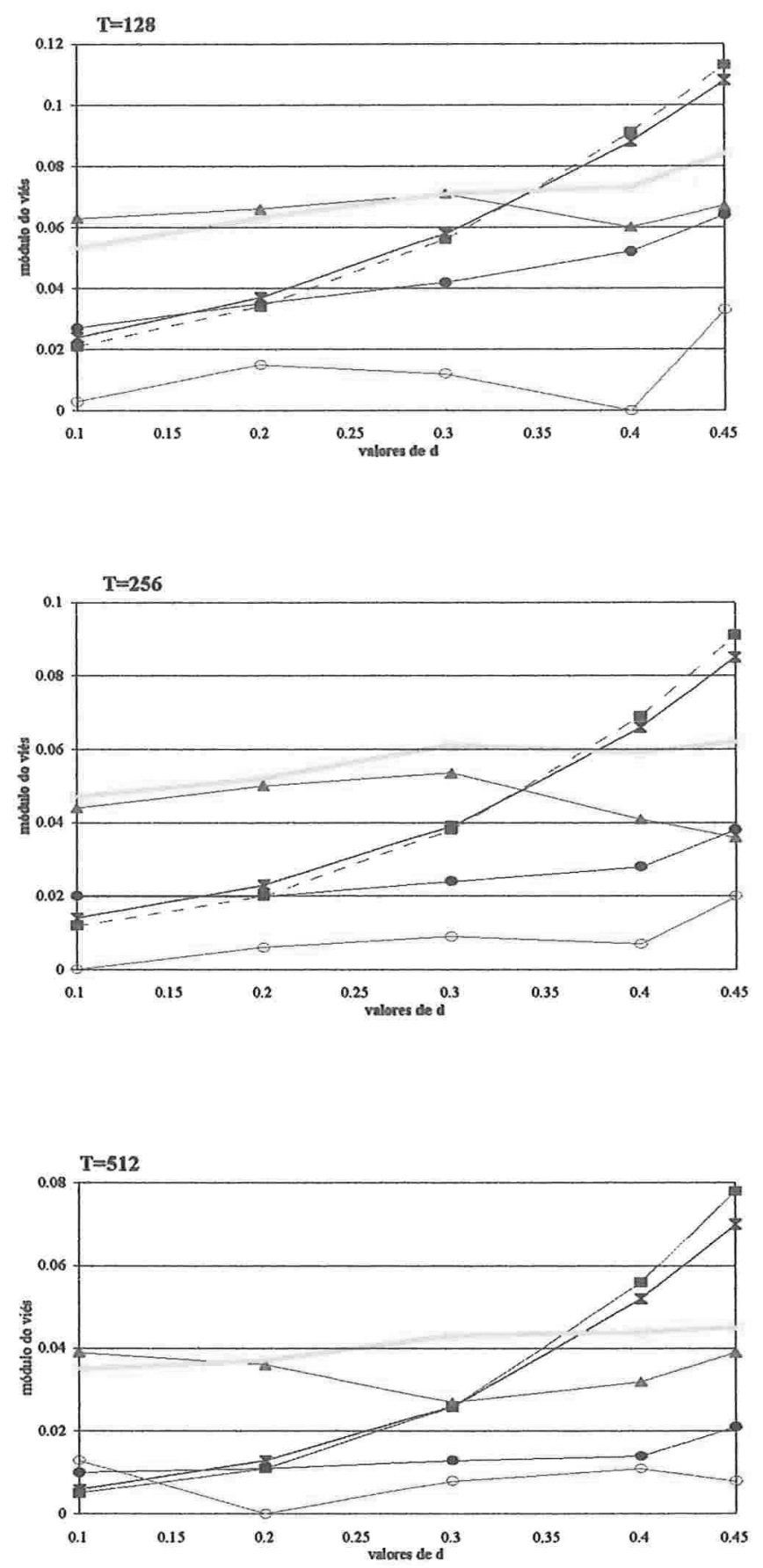

$$
\rightarrow \hat{d}_{c} \rightarrow \hat{d}_{c g} \rightarrow \hat{d}_{p} \triangleleft \hat{d}_{p s} \rightarrow \hat{d}_{m v}-\hat{d}_{w}
$$

Figura 6.1: Variância estimada dos estimadores de $d$ 
A Figura 6.2 compara os estimadores com base na média. em relação a $d$, das variâncias obtidas nas simulaçòes dos cinco modelos ARF I.I/A(0.d.0) para os diferentes tamanhos de amostra. Podemos ver que conforme o tamanho da amostra aumenta, as variâncias dos estimadores diminuem. tornando a diferença entre elas cada vez menor.

A Figura 6.3 apresenta os valores em módulo do viés obtido através das simulaçòes de amostras de tamanho 128 . 256 e 512 , respectivamente. Analisando essa figura percebemos que:

1. Os padrões de comportamento das estimativas de viés em relação aos 5 valores que o parâmetro $d$ assume sào semelhantes para os três tamanhos de amostra. Percebese também que o viés dos estimadores é inversamente proporcional ao tamanho da amostra.

2. O padrão de comportamento entre os estimadores. apesar de não variar em relação ao tamanho da amostra. varia de estimador para estimador. Alguns estimadores se tornam mais viesados quando $d$ se aproxima de 0,5 , que é o caso de $\hat{d}_{c}, \hat{d}_{c g}, \hat{d}_{w}$ e $\hat{d}_{m v}$; os outros não apresentam um comportamento claro do viés em relação aos valores que $d$ assume. que é o caso de $\hat{d}_{p}$ e $\hat{d}_{p s}$.

3. O estimador $\hat{d}_{p}$ é o estimador menos viesado para todos os valore de $d$.

4. Os estimadores $\hat{d}_{c}$ e $\hat{d}_{c g}$ apresentam valores estimativas de viés muito próximas e o mesmo comportamento em relaçào aos valores de $d$. Ao classificar os estimadores segundo o viés esses estimadores estão em segundo lugar quando $d=0,1$ e 0,2 , mas para $d=0,3$ eles passam para a terceira colocação e quando $d=0,4$ e 0,45 eles passam a ser os estimadores mais viesados. Isso se deve a um crescimento muito maior do viés que dos demais estimadores quando o parâmetro $d$ se aproxima do limite de nào estacionariedade.

5. Os estimadores $\hat{d}_{p s}$ e $\hat{d}_{w}$ apresentam estimativas de viés próximas, quando $d=0,1 \mathrm{e}$ 0,2 , aparecendo como os mais viesados. Quando $d$ se aproxima de 0,5 o estimador $\hat{d}_{w}$ apresenta uma tendência de crescimento no viés, o que nào ocorre com $\hat{d}_{p s}$. Com isso, os estimativas de viés desses estimadores passam a se afastar conforme $d$ se aproxima de 0,5 . 


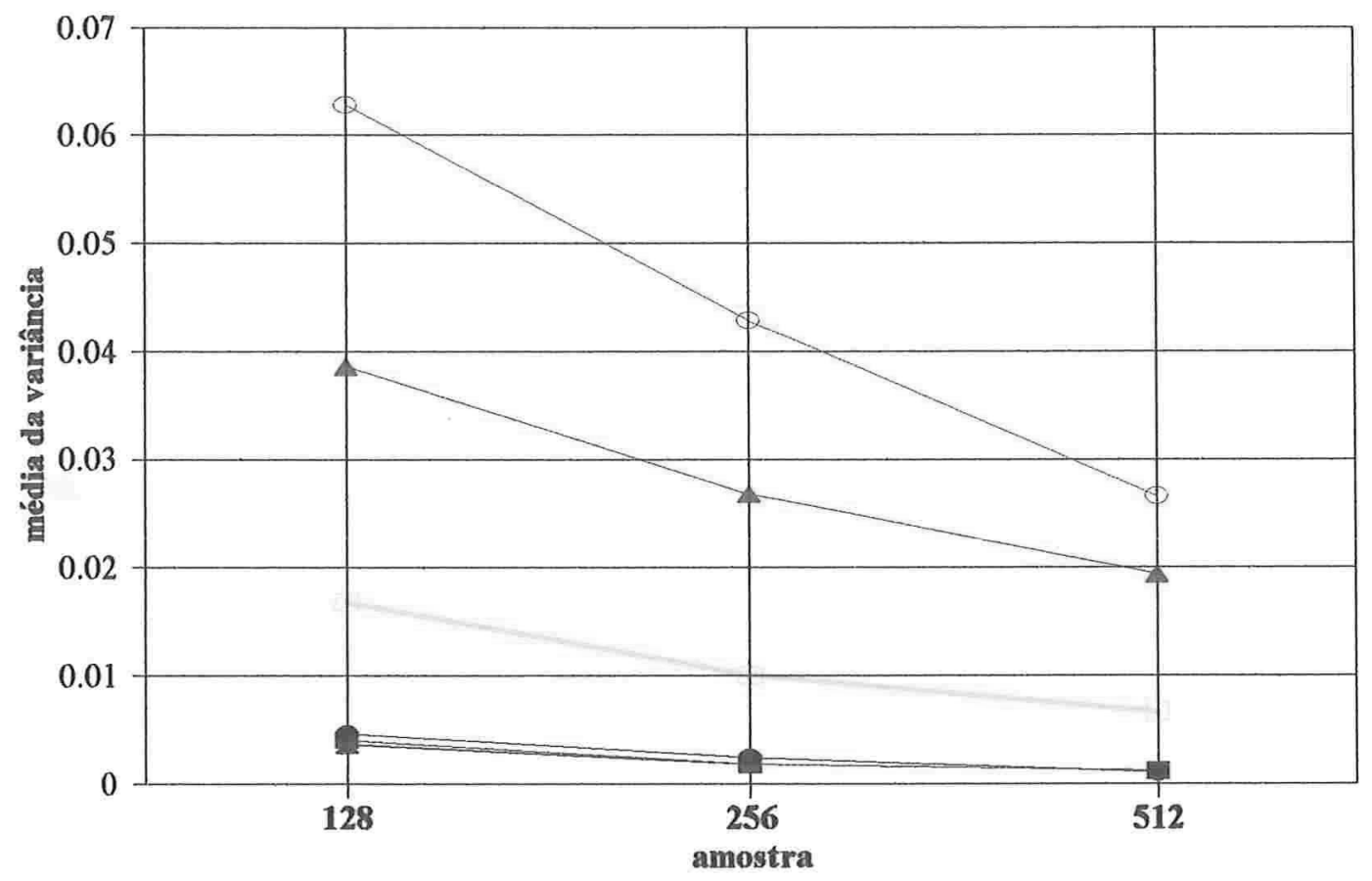

$\rightarrow \hat{d}_{c} \rightarrow \hat{d}_{c g}^{\prime} \quad-\hat{d}_{p} \rightarrow \Delta \hat{d}_{p s} \rightarrow \hat{d}_{m} \rightarrow \hat{d}_{w}$

Figura 6.2: Média das variâncias estimadas, em relação a $d$, dos estimadores. 

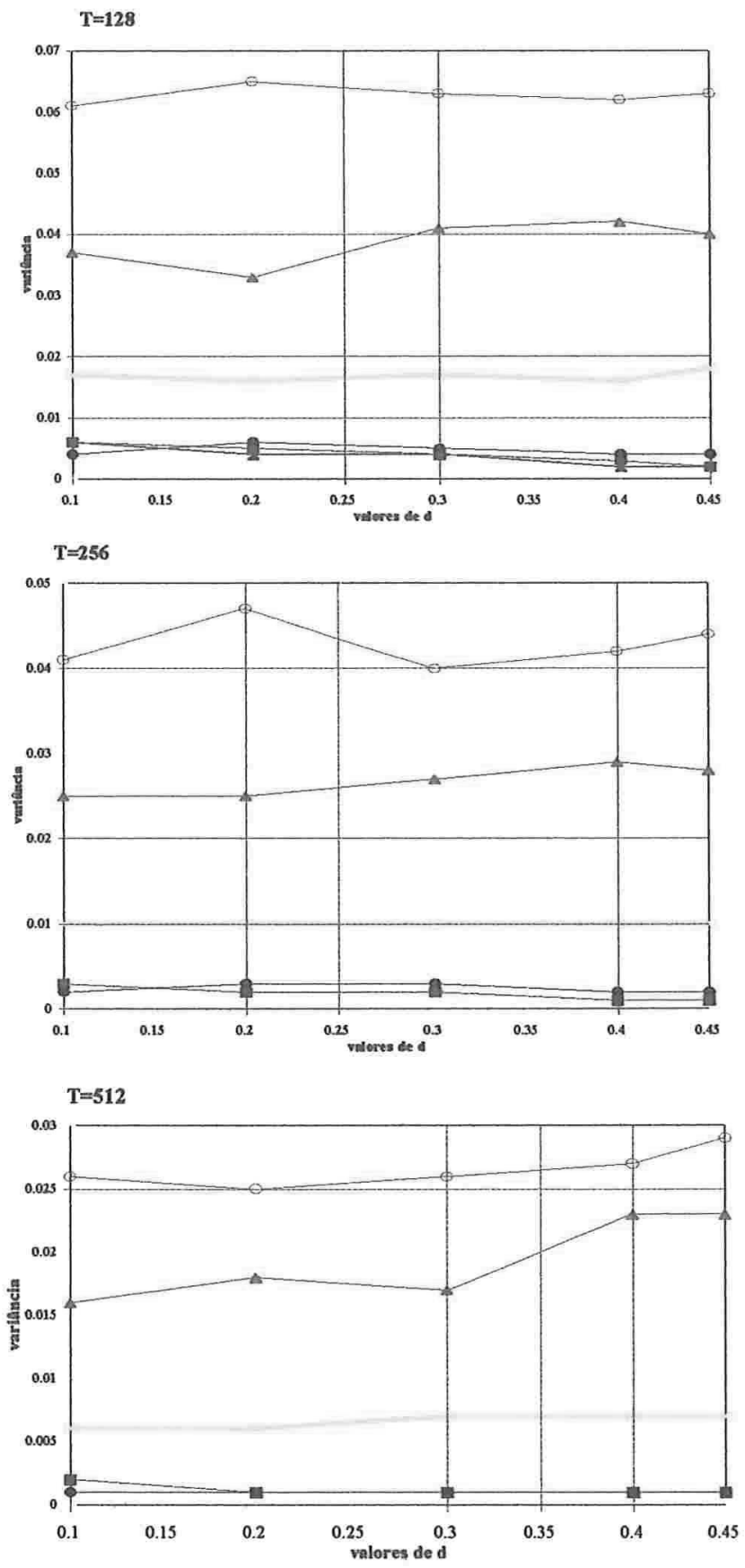

$$
\because \hat{d}_{c} \rightarrow \hat{d}_{c g} \multimap \hat{d}_{p} \multimap \hat{d}_{p s} \multimap \hat{d}_{m v}-\hat{d}_{w}
$$

Figura 6.3: Módulo do viés estimado dos estimadores de $d$ 
6. Devido ao comportamento das estimativas de viés de $\hat{d}_{\mathrm{c}}$ e $\hat{d}_{\mathrm{cg}}$. para $d=0,4$ e 0,45 , os estimadores $\hat{d}_{p s}$ e $\hat{d}_{w}$ passam à terceira e quarta colocaçào. respectivamente.

7. O estimador $\hat{d}_{m v}$ aparece na segunda colocação para todos os valores de $d$.

Resumindo as informaçòes obtidas com as Figuras 6.1 a 6.3 , podemos dizer que:

- A variância estimada, em geral. pouco se altera em relação aos valores que o verdadeiro valor do parâmetro $d$ assume, $d=0,1,0.2,0,3,0,4$. e 0.45 , exceçào feita aos estimadores $\hat{d}_{c}$ e $\hat{d}_{c g}$. Entretanto, a variância sempre diminui com o aumento da amostra.

- As estimativas de viés dos estimadores $\hat{d}_{c}, \hat{d}_{c g} \cdot \hat{d}_{m v}$ e $\hat{d}_{w}$ sofrem um aumento quando o valor de $d$ aumenta, ou seja. quando se aproxima do limite de nào estacionariedade, 0,5 . Quanto aos demais estimadores, nào podemos perceber nenhum comportamento que represente uma tendência no viés em relação aos valores de $d$.

- Em geral, o padrão de comportamento dos viéses estimados dos estimadores, em relação aos valores que $d$ assume. não se altera muito com o aumento do tamanho da amostra, entretanto, eles sofrem uma queda com o aumento da amostra.

- O estimador $\hat{d}_{p}$ foi o menos viesado em todas as situações. com exceção para o caso em que $T=512$ e $d=0,1$, entretanto, possui a maior variância estimada para todos os tamanhos de amostra.

- O estimador $\hat{d}_{m v}$ é um dos menos viesados e apresenta a menor variância para todos os tamanhos de amostra utilizados.

- Os estimadores $\hat{l}_{c}$ e $\hat{l}_{c g}$ apresentaram estimativas das variâncias muito próximas da variância estimada de $\hat{d}_{m v}$ para todos os tamanhos de amostra. Para $d=0,1$ e 0,2 as estimativas de viés destes estimadores estiveram muito próximas do viés estimado de $\hat{d}_{m v}$, mas para $d \geq 0.3$ esses estimadores sofrem um acréscimo muito grande no viés e para $d=0.4$ e 0.45 eles se tornam os mais viesados. Esse comportamento acontece para todos os tamanhos de amostra utilizados. 
- Os estimadores $\hat{d}_{u}$ e $\hat{d}_{p s}$. quando classificados segundo a variância estimada, aparecem em quarto e quinto lugares, respectivamente, para todos os tamanhos de amostra utilizados. Em relaçào ao viés, quando $d$ assume valores próximos de 0,1 esses estimadores aparecem com estimativas de viés muito próximas e são os mais viesados. Mas quando $d$ se aproxima do limite de nào estacionariedade, 0.5 , o estimador $\hat{d}_{w}$ sofre um acréscimo no viés. o que não acontece $\operatorname{com} \hat{d}_{p s}$. C'om isso, as estimativas de viés desses dois estimadores se afastam e, devido ao comportamento de $\hat{d}_{c}$ e $\hat{d}_{c g}$, os estimadores $\hat{d}_{p s}$ e $\hat{d}_{w}$ passam para a terceira e quarta posiçòes, respectivamente.

Para avaliar os estimadores utilizando as informaçòes do viés e da variância conjuntamente. apresentamos os valores do $E \hat{Q} M$. obtidos para todlas as situaçòes estudadas, na Tabela 6.2. Além disso. a Tabela 6.2 dá a classificaçào dos estimadores segundo o EQ̂M médio de cada estimador. que é uma média dos $E \hat{Q} . M^{\prime}$ s obtidos para os cinco valores que o parâmtetro $d$ assume.

Com base nesses resultados, percebemos que existem três grupos de estimadores:

1. $\hat{d}_{m v}, \hat{d}_{c}$ e $\hat{d}_{c g}$, que possuem os menores $E \hat{Q} M^{\prime} s$.

2. $\hat{d}_{w}$, que possui E $\hat{Q} M$ intermediário.

3. $\hat{d}_{p s}$ e $\hat{d}_{p}$, que possuem os maiores $E \hat{Q} M^{\prime} s$.

Observação 1: No grupo 1, citado acima. temos três estimadores. porém eles apresentaram características muito diferentes em relação ao viés.

Observação 2: Os estimadores, $\hat{d}_{c}$ e $\hat{d}_{c g}$ se mostraram muito parecidos. Como foi visto no Capítulo $\tilde{5}, \hat{d}_{c g}$ é uma generalizaçào de $\hat{d}_{c}$, mas pelos resultados das simulaçòes o ganho que se tem com essa generalização nào parece ser significativo. Portanto. levando em consideração a complexidade computacional de $\hat{d}_{c g}$ este deve ser classificado como um estimador menos aconselhável que $\hat{d}_{c}$. 
Tabela 6.2: Classificação dos estimadores pela média do Erro Quadrático Médio Estimado (EQ.M

\begin{tabular}{|c|c|c|c|c|c|c|}
\hline \multirow{2}{*}{$\begin{array}{l}\mathrm{T}=12 \mathrm{~S} \\
\text { Estimador }\end{array}$} & \multicolumn{5}{|c|}{$E \hat{Q} . M(\hat{d})$} & \multirow{2}{*}{$\begin{array}{c}E \hat{Q} M(\hat{d}) \\
\text { Médio }\end{array}$} \\
\hline & $\mathrm{d}=0,1$ & $\mathrm{~d}=0.2$ & $\mathrm{~d}=0.3$ & $\mathrm{~d}=0,4$ & $\mathrm{~d}=0,45$ & \\
\hline$\hat{d}_{m v}$ & 0.005 & $0,00 \bar{\imath}$ & 0.007 & $0,00 \tau$ & 0,008 & 0.007 \\
\hline$\hat{d}_{c}$ & 0.007 & 0,006 & 0,007 & 0.010 & 0,014 & 0.009 \\
\hline$\hat{d}_{c g}$ & 0.006 & 0.006 & 0,007 & 0.011 & 0.015 & 0.009 \\
\hline$\hat{d}_{u}$ & 0.020 & 0.020 & 0.022 & 0,022 & 0.026 & 0.022 \\
\hline$\hat{d}_{p s}$ & 0.041 & 0.037 & 0.046 & 0.046 & 0.045 & 0.043 \\
\hline$\hat{d}_{p}$ & 0.062 & 0.065 & $0.06: 3$ & 0.062 & 0.064 & 0.063 \\
\hline$t=256$ & \multicolumn{5}{|c|}{$E \hat{Q} . M(\hat{d})$} & $E \hat{Q} . M(\hat{d})$ \\
\hline Estimador & $\mathrm{d}=0,1$ & $\mathrm{~d}=0,2$ & $\mathrm{~d}=0.3$ & $\mathrm{~d}=0,4$ & $\mathrm{~d}=0.45$ & Médio \\
\hline$\hat{d}_{m v}$ & 0.003 & 0,003 & $0,00: 3$ & $0,00: 3$ & 0,003 & $0,00: 3$ \\
\hline$\hat{d}_{c}$ & 0,003 & 0,003 & 0,003 & 0,006 & 0,008 & 0,005 \\
\hline$\hat{d}_{c g}$ & 0.003 & 0,003 & $0,00: 3$ & 0,006 & 0,010 & 0.005 \\
\hline$\hat{d}_{w}$ & 0.011 & 0,011 & 0,013 & 0.012 & 0,013 & 0.012 \\
\hline$\hat{d}_{p s}$ & 0.027 & 0,028 & $0,0: 30$ & $0,0: 31$ & $0,0: 30$ & 0,029 \\
\hline$\hat{d}_{p}$ & 0.041 & $0,04 i$ & 0,040 & 0.042 & 0.044 & 0.043 \\
\hline $\mathrm{T}=512$ & \multicolumn{5}{|c|}{$E \hat{Q} M(\hat{d})$} & $E \hat{Q} M(\hat{d})$ \\
\hline Estimador & $\mathrm{d}=0,1$ & $\mathrm{~d}=0.2$ & $d=0,3$ & $\mathrm{~d}=0,4$ & $\mathrm{~d}=0,45$ & Médio \\
\hline$\hat{d}_{m v}$ & 0.001 & 0,001 & 0.001 & 0,001 & 0,002 & 0.001 \\
\hline$\hat{d}_{c}$ & 0.002 & 0,001 & 0.002 & 0.004 & 0.006 & 0,003 \\
\hline$\hat{d}_{c \mathfrak{l g}}$ & 0.002 & 0,001 & 0,002 & 0.004 & 0.007 & 0.003 \\
\hline$\hat{d}_{w}$ & 0.006 & 0.006 & $0,00 \tau$ & $0.00 \tau$ & 0.007 & 0.007 \\
\hline$\hat{d}_{p s}$ & 0.018 & 0,020 & 0,017 & 0,024 & 0,024 & 0.021 \\
\hline$\hat{d}_{p}$ & 0.026 & 0.025 & 0.026 & 0.027 & 0,029 & 0,027 \\
\hline
\end{tabular}




\subsection{Resultados para os modelos $\operatorname{ARFIMA}(1, \mathrm{~d}, 0)$}

Vesta seção apresentamos os resultados obtidos com as simulaçòes de amostras do processo $A R F I M A(1, d .0), \operatorname{com} d=0.3$ e $o_{1}=0 . \bar{\tau}$. onde $\omega_{1}$ é o parâmetro auto-regressivo de ordem 1. Com isso, analisaremos apenas um tipo de modelo $A R F I M A(1, d, 0)$. Com base nessas simulaçòes, podemos avaliar como os estimadores de $d$ se comportam quando a série de longa memória também apresenta comportamento auto-regressivo de primeira ordem. Ou seja, queremos saber se as conclusòes obtidas a respeito dos estimadores de $d$ no caso do modelo $A R F I M A(1, d, 0)$ serào semelhantes as obtidas no caso do modelo $A R F I M A(0, d, 0)$. Também temos interesse em comparar as estimativas obtidas para $O_{1}$ nos diversos métodos de estimação. pois a estimativa de $o_{1}$ depende do estimador de $d$ utilizado no método de estimaçào dos parâmetros do modelo . ARF I.M.A(1.d.0).

C'omo foi visto na Seção 5.2.2, o estimador de $\phi_{1}$ depende do estimador de $d$ utilizado no método de estimação. Por isso, denotaremos por $\hat{\phi}_{c}, \hat{O}_{c g}, \hat{O}_{p}, \hat{\phi}_{p s}, \hat{O}_{m v}$ e $\hat{\phi}_{w}$ os estimadores de $\phi_{1}$ relacionados aos estimadores $\hat{d}_{c}, \hat{d}_{c g}, \hat{d}_{p}, \hat{d}_{p s}, \hat{d}_{m v}$ e $\hat{d}_{w}$, respectivamente.

Foram utilizados três tamanhos de amostras nas simulaçòes, T=128, 256 e 512. A Tabela 6.3 apresenta a variância e o viés estimados de $\hat{d}$ e $\hat{O}_{1}$, obtidos para os seis métodos de estimaçào estudados. Para melhor comparar os estimadores apresentamos alguns gráficos que resumem os resultados da Tabela 6.3. A Figura 6.4 apresenta o gráfico das variâncias estimadas dos estimadores de $d(\hat{\operatorname{Var}}(\hat{d}))$. dadas por $(6.1)$, para cada tamanho de amostra utilizado nas simulaçǒes. Com base nesses resultados, podemos dizer que:

1. As variâncias estimadas de $\hat{d}_{c}$. $\hat{l}_{c g}$ e $\hat{d}_{m v}$ são as menores para todos os tamanhos de amostra utilizados.

2. O estimador $\hat{d}_{p}$ apresenta a maior estimativa de variância para todos os tamanhos de amostra; os estimadores $\hat{d}_{w}$ e $\hat{d}_{p s}$ também têm um comportamento ruim quando comparados aos melhores.

3. Podemos observar que, para todos os estimadores, as variâncias encontradas diminuem quando aumentamos o tamanho da amostra. 
Tabela 6.3 - Resultados de uma simulação, com 1000 réplicas, de um modelo $\operatorname{ARFIMA(1,d,0).~}$

\begin{tabular}{|c|c|c|c|c|c|}
\hline \multirow{2}{*}{$\begin{array}{l}\mathrm{T}=12 \mathrm{~S} \\
\text { Estimador }\end{array}$} & \multicolumn{2}{|c|}{$\mathrm{d}=0.3$} & \multirow[b]{2}{*}{ Estimador } & \multicolumn{2}{|c|}{$o_{1}=0 . \bar{\imath}$} \\
\hline & $\hat{\operatorname{Var}(\hat{d})}$ & $\hat{B}(\hat{d})$ & & $\hat{\operatorname{Var}\left(\hat{O}_{1}\right)}$ & $\hat{B}\left(\hat{\phi}_{1}\right)$ \\
\hline$\hat{d}_{c}$ & 0,003 & $-0,07 \mathrm{~S}$ & $\hat{\phi}_{c}$ & 0,001 & 0,121 \\
\hline$\hat{d}_{c y}$ & 0,003 & $-0,079$ & $\hat{\dot{O}}_{c g}$ & 0.001 & 0.122 \\
\hline$\hat{d}_{p}$ & 0,078 & 0,2333 & $\hat{O}_{p}$ & 0.007 & 0.015 \\
\hline$\hat{d}_{p s}$ & $0.04 \pi$ & 0.149 & $\hat{O}_{p s}$ & 0.006 & 0.033 \\
\hline$\hat{d}_{m v}$ & $0.00 \uparrow$ & $-0,148$ & $\hat{O}_{m v}$ & 0.006 & 0.082 \\
\hline$\hat{d}_{w}$ & 0,023 & -0.008 & $\hat{\phi}_{w}$ & $0.00: 3$ & 0.092 \\
\hline $\mathrm{T}=256$ & \multicolumn{2}{|c|}{$\mathrm{d}=0.3$} & & \multicolumn{2}{|c|}{$\phi_{1}=0.7$} \\
\hline Estimador & $\hat{\operatorname{Var}}(\hat{d})$ & $\hat{B}(\hat{d})$ & Estimador & $\hat{\operatorname{lar}\left(\hat{\phi}_{1}\right)}$ & $\hat{B}\left(\hat{\phi}_{1}\right)$ \\
\hline$\hat{d}_{c}$ & 0,001 & $-0,063$ & $\hat{\phi}_{c}$ & 0,001 & 0,122 \\
\hline$\hat{d}_{c g}$ & 0,002 & $-0,066$ & $\hat{\phi}_{c g}$ & 0,001 & 0,121 \\
\hline$\hat{d}_{p}$ & 0,046 & 0,139 & $\hat{o}_{p}$ & 0.006 & 0,045 \\
\hline$\hat{d}_{p s}$ & $0,02 \bar{\imath}$ & 0,086 & $\hat{\phi}_{p s}$ & 0.004 & 0,059 \\
\hline$\hat{d}_{m v}$ & 0,003 & $-0,122$ & $\hat{\dot{O}}_{m v}$ & $0,00: 3$ & 0,076 \\
\hline$\hat{d}_{w}$ & 0,014 & $-0,021$ & $\hat{\phi}_{w}$ & 0,002 & 0,103 \\
\hline $\mathrm{T}=512$ & \multicolumn{2}{|c|}{$\mathrm{d}=0.3$} & & \multicolumn{2}{|c|}{$\phi_{1}=0 . \bar{\imath}$} \\
\hline Estimador & $\hat{\operatorname{Var}}(\hat{d})$ & $\hat{B}(\hat{d})$ & Estimador & $\hat{\operatorname{Var}\left(\hat{\phi}_{1}\right)}$ & $\hat{B}\left(\hat{\phi}_{1}\right)$ \\
\hline$\hat{d}_{c}$ & 0,001 & $-0,053$ & $\hat{\phi}_{c}$ & 0,000 & 0,120 \\
\hline$\hat{d}_{c g}$ & 0,001 & $-0,056$ & $\hat{\phi}_{c g}$ & 0,000 & 0,121 \\
\hline$\hat{d}_{p}$ & 0,029 & 0.070 & $\hat{O}_{p}$ & 0,004 & 0,071 \\
\hline$\hat{d}_{p s}$ & 0,019 & 0.033 & $\hat{\partial}_{p s}$ & $0,00: 3$ & 0,083 \\
\hline$\hat{d}_{m v}$ & 0,002 & $-0,084$ & $\hat{\varphi}_{m v}$ & 0,002 & 0,058 \\
\hline$\hat{d}_{w}$ & 0,008 & $-0,031$ & $\hat{\phi}_{w}$ & 0,001 & 0,108 \\
\hline
\end{tabular}




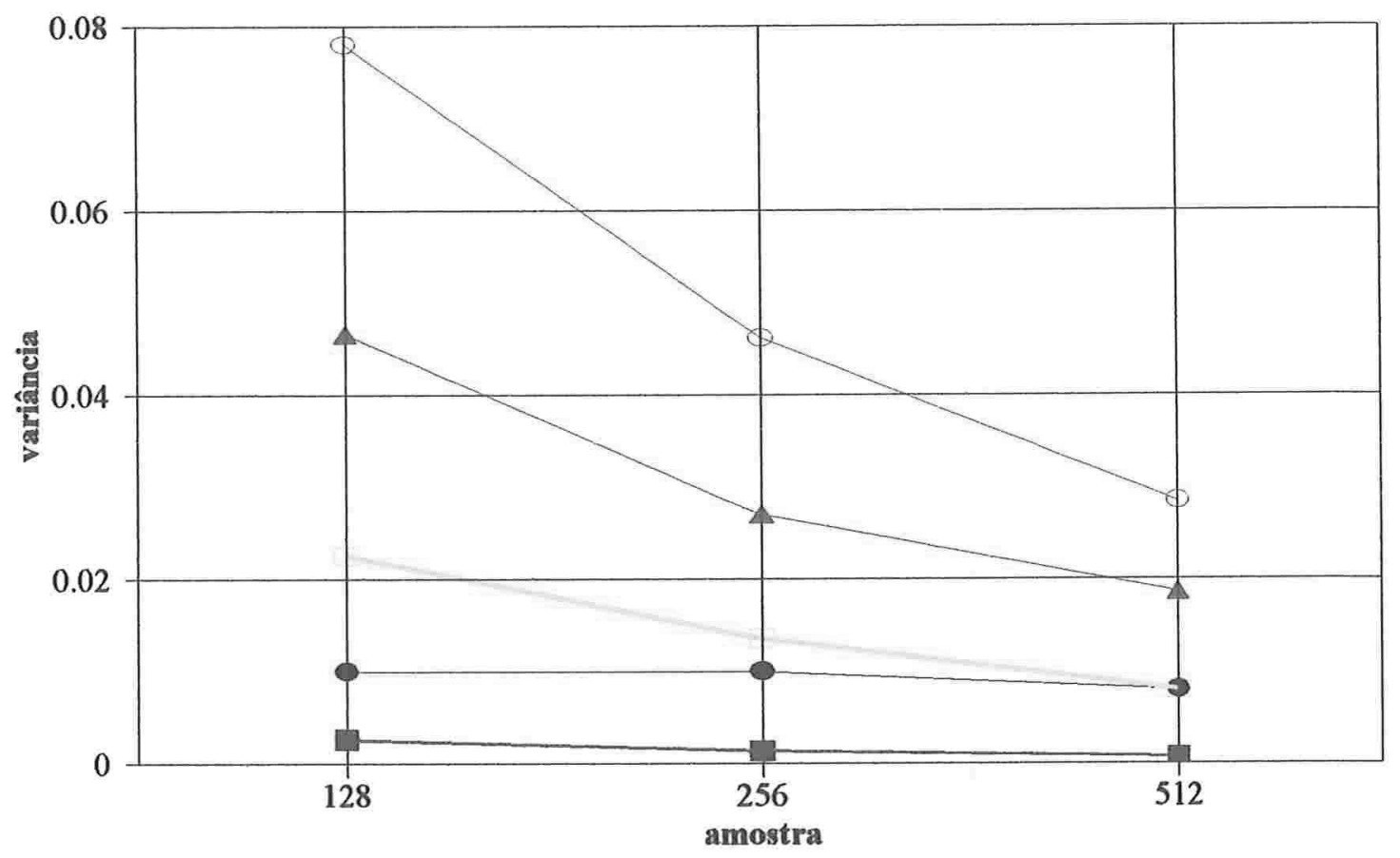

$\rightarrow \hat{d}_{c} \rightarrow \hat{d}_{c g} \quad-\hat{d}_{p} \rightarrow \hat{d}_{p s}-\hat{d}_{m v} \longrightarrow \hat{d}_{w}$

Figura 6.4: Variância estimada dos estimadores de $d$. 
A Figura 6.5 apresenta um gráfico que compara os estimadores do parâmetro $d$ com base nos valores absolutos das estimativas de viés dos estimadores de $d(\hat{B}(\hat{d}))$. dadas por $(6.2)$. Com base nesse gráfico. temos que:

1. O estimador $\hat{d}_{w}$ é o menos viesado para todos os tamanhos de amostra utilizados . mas seu viés aumenta (em valores absolutos) quando aumentamos o tamanho da amostra.

2. Os estimadores $\hat{d}_{c}$ e $\hat{d}_{c g}$ aparecem com estimativas de viés muito próximas, além de serem os segundos menos viesados para amostras de tamanho $T=128$ e 256. Para amostras de tamanho 512 esses estimadores passam a ser os terceiros menos viesados, isso devido ao rápido decaimento do viés de $\hat{d}_{p s}$. No caso desses estimadores. o viés diminui com o aumento do tamanho da amostra.

3. Os estimadores $\hat{d}_{m \varepsilon}$, $\hat{d}_{p s}$ e $\hat{d}_{p}$ nào têm um bom comportamento com relação ao viés estimado de $d$. Nota-se, entretanto, que o valor do viés decai com o aumento do tamanho da amostra.

4. Podemos observar que com o aumento do tamanho da amostra as distâncias entre as estimativas de viés dos estimadores estudados diminuem muito.

A Figura 6.6 apresenta um gráfico das variâncias estimadas dos estimadores de $\phi_{1}$ $\left(\hat{\operatorname{Var}}\left(\hat{\phi}_{1}\right)\right)$, dadas por (6.1). Como foi dito antes, denotaremos por $\hat{\phi}_{c}, \hat{\phi}_{c g}, \hat{\phi}_{p}, \hat{\phi}_{p s}, \hat{\phi}_{m v}$ e $\hat{\phi}_{w}$ os estimadores de $o_{1}$ relacionados aos estimadores $\hat{d}_{c}, \hat{d}_{c g}, \hat{d}_{p}, \hat{d}_{p s}, \hat{d}_{m v}$ e $\hat{d}_{w}$, respectivamente. Sendo assim, com base nos resultados, temos que:

1. As variâncias estimadas de $\hat{\phi}_{c}$ e $\hat{\phi}_{c g}$ estão muito próximas. além de serem os estimadores que apresentam as menores variâncias para todos os tamanhos de amostra utilizados.

2. O estimador $\hat{o}_{w}$ apresenta a segunda menor estimativa de variância para todos os tamanhos de amostra.

3. O estimador $\hat{\phi}_{m v}$ tem um comportamento ruim para $\mathrm{T}=12 \mathrm{~S}$ e 256 .

4. O estimador $\hat{\phi}_{p}$ é o pior dentre eles, apresentando a maior variância para todos os tamanhos de amostra utilizados. 


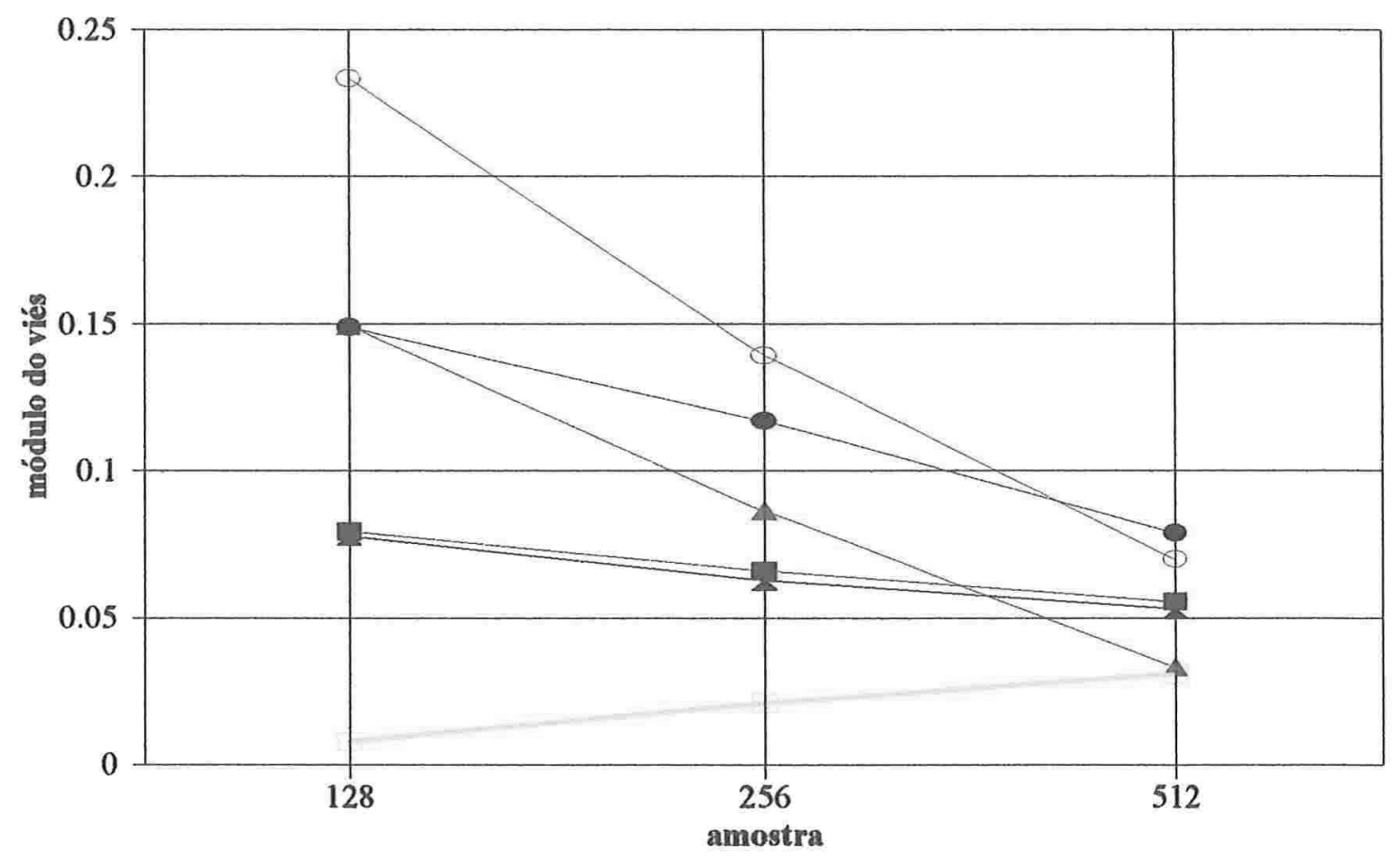

$\varangle \quad \hat{d}_{c} \rightarrow \hat{d}_{c g} \odot \hat{d}_{p} \rightarrow \hat{d}_{p s} \multimap-\hat{d}_{m v} \rightarrow \hat{d}_{w}$

Figura 6.5: Módulo do viés estimado dos estimadores de $d$. 


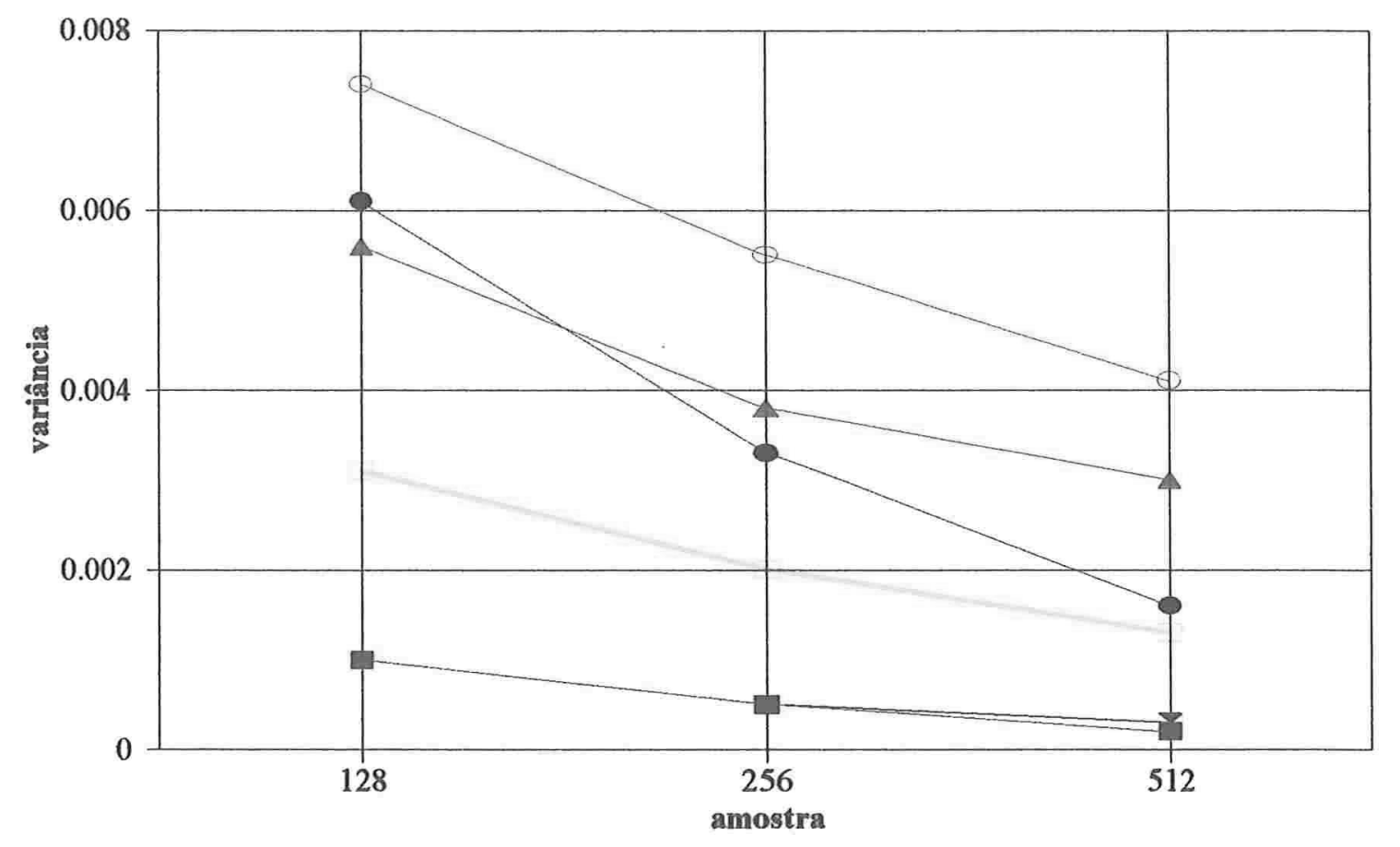

$\mp \hat{\phi}_{c} \rightarrow \hat{\phi}_{c g}-\hat{\phi}_{p} \rightarrow \hat{\phi}_{p s} \rightarrow \hat{\phi}_{m v} \rightarrow \hat{\phi}_{w}$

Figura 6.6: Variância estimada dos estimadores de $\phi_{1}$. 
5. Também podemos observar que. para todos os estimadores. as variàncias estimadas de o diminuem quando aumentamos o tamanho da amostra.

6. O estimador $\hat{O}_{m v}$ apresenta o maior decaimento da rariância com o aumento da amostra. Já os demais estimadores se assemelham em relaçào ao decaimento da variância quando aumentamos o tamanho da amostra.

A Figura 6.7 apresenta um gráfico que compara os estimadores com base nas estimativas do valor absoluto do viés dos estimadores de $o_{1}\left(\hat{B}\left(\hat{O}_{1}\right)\right)$ para todos os tamanhos de amostra utilizados. C'om base neste gráfico. podemos observar que:

1. O estimador $\hat{o}_{p}$ é o menos viesado para $T=128$ a 256 . mas para $T=512$ troca de posiçào com $\hat{O}_{m u}$ e passa a ser o segundo menos viesado. Além disso. o viés deste estimador aumenta quando aumentamos o tamanho da amostra.

2. O estimador $\hat{O}_{p s}$ é o segundo menos viesado para $T=128$ e 256 . mas para $T=512$ passa a ser o terceiro menos viesado. Entretanto. seu viés também aumenta quando aumentamos o tamanho da amostra.

3. O estimador $\hat{O}_{m}$ aparece como o terceiro menos viesado para amostras de tamanho 128 e 256 . mas para $T=512$ ele passa a ser o estimador menos viesado. Além disso, o viés deste estimador é o único que diminui quando aumentamos o tamanho da amostra.

4. O estimador $\hat{O}_{w}$ é o quarto menos viesado para todos os tamanhos de amostra e seu viés aumenta com o aumento da amostra.

5. Os estimadores $\hat{\partial}_{\mathrm{c}}$ e $\hat{O}_{\varepsilon g}$ apresentam valores próximos para o viés e sào os mais viesados para todos os tamanhos de amostra utilizados. Além disso. os valores do viés desses estimadores permanecem estáveis com o aumento do tamanho da amostra.

Para avaliar os métodos de estimaçào é necessário analisar conjuntamente o comportamento das estimativas dos parâmetros $d$ e $o_{1}$. Primeiro ramos analisar, com base nas estimativas do viés e da variância. como os diversos estimadores de d e o se comportaram com o aumento da amostra. Entào. em relaçào a essa questào podemos dizer que quando aumentamos o tamanho da amostra: 


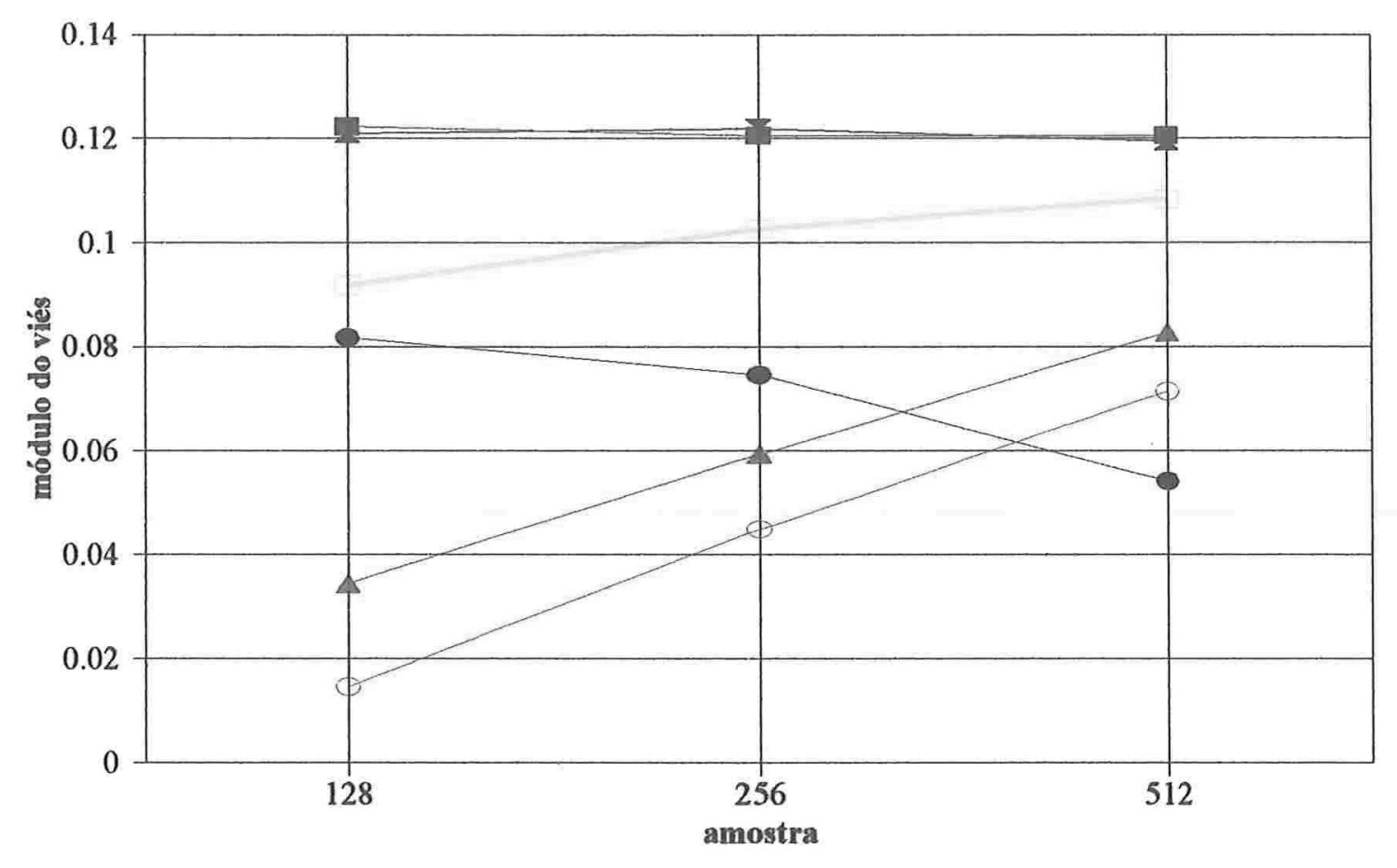

$\geq \hat{\phi}_{c} \rightarrow \hat{\phi}_{c g}-\hat{\phi}_{p} \rightarrow \hat{\phi}_{p s} \rightarrow \hat{\phi}_{m v} \longrightarrow \hat{\phi}_{w}$

Figura 6.7: Módulo do viés estimado dos estimadores de $\phi_{1}$. 
1. A variância estimada de todos os estimadores de de o diminuem.

2. Tanto o viés estimado de $\hat{d}_{n}$ (quanto o de $\hat{\partial}_{w}$ aumentan. entretanto. eles diminuem no caso dos estimadores $\hat{d}_{m e}$ e $\hat{o}_{m}$.

3. Os valores dos vieses estimados de $\hat{d}_{p}$ e de $\hat{d}_{p s}$ dimimuem. enquanto os de $\hat{o}_{p}$ e $\hat{\phi}_{p s}$ aumentam.

4. Com relaçào aos estimadores $\hat{d}_{\varepsilon}$ e $\hat{d}_{\imath g}$. podemos observar que as estimativas dos vieses diminuem. enquanto as de $\hat{O}_{c}$ e $\hat{O}_{c g}$ pouco se alteram.

Agora. iremos comparar os métodos de estimaçào analisando conjuntamente os estimadores de d e de $O_{1}$. Desta forma. temos que:

1. Os estimadores $\hat{d}_{u}$. $\hat{d}_{c}$ e $\hat{d}_{c y}$ sào os menos viesados. enculanto $\hat{O}_{u}$. $\hat{\partial}_{c}$ e $\hat{\phi}_{c g}$ são os mais viesados. Entretanto. todos esses estimadores possuem as menores estimativas para a variâncias.

2. O estimador $\hat{d}_{p}$ apresenta a maior estimativa da variância e é um dos mais viesados. Com relação a $\hat{o}_{p}$, este é um dos menos viesados. mas ć o que possui a maior variância.

3. O estimador $\hat{d}_{m v}$ apresenta uma das menores estimativas de variância, mas é um dos mais viesados. Os valores das estimativas da variância c do viés de $\hat{\phi}_{m v}$ são intermediários quando comparados aos de outros estimadores. mas para $\mathrm{T}=512$, este se torna o estimador menos viesado.

4. O estimador $\hat{d}_{p s}$ é um dos mais viesados para amostras pequenas, mas a estimativa do viés cai rapidamente quando aumentamos o tamanho da amost ra fazendo com que ele se torne um dos menos viesados para $T=512$. Entretanto. cle apresenta uma das maiores estimativas para a variância. $\hat{O}_{p s}$ possui uma clas maiores estimativas de variância e valores intermediários para as estimativas de riés.

C'om base nessas análises. podemos observar que muitas vezes um estimador menos viesado possui uma variância alta e vice-versa. o que dificulta nossa análise. Para conseguir classificar os estimadores segundo essas duas medidas conjuntamente. usaremos o erro quadrático médio estimado dos estimadores de $d(E \hat{Q} . M(\hat{d}))$ e dos estimadores de $\phi_{1}\left(E \hat{Q} M\left(\hat{\phi}_{1}\right)\right)$, 
clados por (6.3). A seguir. apresentamos a Tabela 6.t com a classificaçào dos estimadores de d e $o_{1}$. de acordo com os valores do $E Q . M$ desses estimadores. para os tamanhos de amostra. $T=128.256$ e 512. Com base nesta tabela. percebemos que a classificaçào dos estimadores de $d$ permanece a mesma. enquanto a classificaçào dos estimadores de o difere para cada tamanho de amostra utilizado. Entretanto. as diferenças entre os EQ.$M^{\prime}$ 's dos estimadores de $o_{1}$ são bem menores que as dos estimadores de $d$. indicando que pequenas variações do EQ. M podem fazer com que a classificaçào dos estimadores de $O_{1}$ mude. Outro fato rapidamente observado é que os métodos de estimação que possuem os menores $E \hat{Q} M^{\prime}$ s de $\hat{d}$ são aqueles que possuem os maiores $E \hat{Q} M^{\prime}$ 's de $O_{1}$ e vice-rersa.

('om base nessas observaçoes. podemos dizer que para classificar os métodos devemos nos basear na classificaçào dos estimadores de d pelo EQQ.YI pois. de acordo com o EQQM. os estimadores de or sào muito semelhantes. Sendo assim. concluńmos que:

1. Os métodos da autocorrelaçào e da autocorrelaçào generalizado sào os melhores métodos de estimação, além de serem muito semelhantes. Eles apresentam os menores $E \hat{Q} M^{\prime} s$ para os estimadores de $d$ e os maiores $E \hat{Q} . M^{\prime}$ s para os estimadores de $O_{1}$

2. O método das ondaletas é classificado como o segundo melhor método, apresentando o segundo menor valor do EQQM dos estimadores de d e valores intermediários para os $E \hat{Q} \cdot M^{\prime} s$ dos estimadores de $\phi_{1}$.

3. O método de máxima verossimilhança é o terceiro melhor método de estimação, $\hat{d}_{m v}$ apresenta o terceiro menor valor do $E \hat{Q} M$ e $\hat{O}_{m \text { r }}$ apresenta valores intermediários dos $E \hat{Q} \cdot M^{\prime} s$, para $\mathrm{T}=128$ e 256 . e o menor valor para $\mathrm{T}=512$.

4. Os métodos baseados no periodograma sào os piores. apresentando os maiores valores do $E \hat{Q} . M(\hat{d})$ e os menores valores do $E \hat{Q} . M(\hat{o})$ 
Tabela 6.4: Classificação dos estimadores de $d$ e O pelo EQ. II

\begin{tabular}{|c|c|}
\hline \multirow{2}{*}{$\begin{array}{l}\mathrm{T}=128 \\
\text { Estimador }\end{array}$} & $d=0.3$ \\
\hline & $E \hat{Q} . M(\hat{d})$ \\
\hline$\hat{d}_{c}$ & 0.009 \\
\hline$\hat{d}_{c g}$ & 0.009 \\
\hline$\hat{d}_{u}$ & $0.0 \cdot 2: 3$ \\
\hline$\hat{d}_{m e}$ & 0.032 \\
\hline$\hat{d}_{p s}$ & 0.069 \\
\hline$\hat{d}_{p}$ & 0.132 \\
\hline \multirow{2}{*}{$\begin{array}{l}T=256 \\
\text { Estimador }\end{array}$} & $\mathrm{d}=0.3$ \\
\hline & $E \hat{Q} M(\hat{d})$ \\
\hline$\hat{d}_{c}$ & 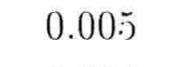 \\
\hline$\hat{d}_{c y}$ & 0.006 \\
\hline$\hat{d}_{n}$ & 0.014 \\
\hline$\hat{d}_{m x}$ & 0.025 \\
\hline$\hat{d}_{p s}$ & 0.035 \\
\hline$\hat{d}_{p}$ & 0.066 \\
\hline \multirow{2}{*}{$\begin{array}{l}\mathrm{T}=512 \\
\text { Estimador }\end{array}$} & $\mathrm{d}=0.3$ \\
\hline & $E \hat{Q} M(\hat{d})$ \\
\hline$\hat{d}_{c}$ & 0.004 \\
\hline$\hat{d}_{c g}$ & 0.004 \\
\hline$\hat{d}_{u}$ & 0.009 \\
\hline$\hat{d}_{m u}$ & 0.015 \\
\hline$\hat{d}_{p s}$ & 0.020 \\
\hline$\hat{d}_{p}$ & 0.0333 \\
\hline
\end{tabular}

\begin{tabular}{|c|c|}
\hline \multirow{2}{*}{$\begin{array}{l}T=128 \\
\text { Estimador }\end{array}$} & $o_{1}=0 . \bar{\imath}$ \\
\hline & $E \hat{Q} \cdot M /\left(\hat{O}_{1}\right)$ \\
\hline$\hat{O}_{p s}$ & $0.00 \tau$ \\
\hline$\hat{O}_{p}$ & 0.008 \\
\hline$\hat{O}_{u}$ & 0.012 \\
\hline$\hat{O}_{m u}$ & $0.01: 3$ \\
\hline$\hat{O}_{e}$ & 0.016 \\
\hline$\hat{O}_{e y}$ & 0.016 \\
\hline \multirow{2}{*}{$\begin{array}{l}\mathrm{T}=256 \\
\text { Estimador }\end{array}$} & $o_{1}=0 . \bar{\imath}$ \\
\hline & $E \hat{Q} \cdot M\left(\hat{O}_{1}\right)$ \\
\hline$\hat{O}_{p s}$ & 0.007 \\
\hline$\hat{O}_{p}$ & 0.008 \\
\hline$\hat{O}_{m e}$ & 0.012 \\
\hline$\hat{O}_{u}$ & $0.01: 3$ \\
\hline$\hat{O}_{c g}$ & 0.015 \\
\hline$\hat{O}_{\mathrm{e}}$ & $0.01 \overline{5}$ \\
\hline \multirow{2}{*}{$\begin{array}{l}\mathrm{T}=512 \\
\text { Estimador }\end{array}$} & $o_{1}=0 . \bar{\imath}$ \\
\hline & $E Q . M\left(\hat{O}_{1}\right)$ \\
\hline$O_{m}$ & 0.008 \\
\hline$\hat{O}_{p}$ & 0.009 \\
\hline$\hat{O}_{p s}$ & 0.010 \\
\hline$\hat{O}_{11}$ & $0.01: 3$ \\
\hline$\hat{O}_{i}$ & 0.015 \\
\hline$\hat{O}_{0 y}$ & 0.015 \\
\hline
\end{tabular}




\subsection{Resultados para o modelo $\operatorname{ARFIMA}(0, \mathrm{~d}, 1)$}

Nesta seçào apresentamos os resultados das simulaçòes de amostras do processo $A R F I . M A(0 . d .1) \mathrm{com} d=0.3$ e $\theta_{1}=-0.6$. onde $\theta_{1}$ é o parâmetro de médias móveis de ordem um. Neste caso, também foram simuladas 1.000 amostras deste processo utilizando 3 tamanhos de amostras diferentes. $T=128$. 256 e 512 . Da mesma forma que o estimador de $o_{1}$ no processo $A R F I M A(1, d, 0)$. o estimador de $\theta_{1}$ depende do método utilizado para estimar o parâmetro $d$. Com isso. denotaremos por $\hat{\theta}_{\varepsilon} . \hat{\theta}_{\varepsilon g} . \hat{\theta}_{p} . \hat{\theta}_{p s}, \hat{\theta}_{m v}$ e $\hat{\theta}_{w}$ os estimadores de $\theta_{1}$ relacionados aos estimadores de $\hat{d}_{c}, \hat{d}_{c y}, \hat{d}_{p}, \hat{d}_{p s}, \hat{d}_{m v}$ e $\hat{d}_{w}$. respectivamente.

Para analisar os diversos métodos de estimaçào. utilizaremos as estimativas da variância. do viés e do erro quadrático médio dos estimadores de de de $\theta_{1}$. darlos por $(6.1),(6.2)$ e (6.3). respectivamente.

A Tabela 6.j apresenta o viés e a variância estimados dos estimadores de $d$ e de $\theta_{1}$ para os três tamanhos de amostra utilizados. Apresentaremos alguns gráficos baseados nesta tabela que ajudam na interpretaçào desses resultados.

A Figura 6.8 apresenta o gráfico da variância estimada dos estimadores de $d$ para os três tamanhos de amostra. Com base neste gráfico. temos que:

1. As variâncias estimadas de todos os estimadores de d diminuem com o aumento do tamanho da amostra. fazendo com que as diferenças entre essas variâncias diminuam.

2. Os estimadores $\hat{d}_{c} \cdot \hat{d}_{c g}$ e $\hat{d}_{m v}$ apresentam as menores estimativas da variância.

3. Os estimadores $\hat{d}_{u}$ e $\hat{d}_{p s}$ possuem a segunda e a terceira menor estimativa de variância. respectivamente. Entretanto, para $\mathrm{T}=512$ suas estimativas praticamente se igualam.

4. O estimador $\hat{d}_{p}$ é o estimador que oferece a maior estimativa de variância. 
Tabela 6.5 - Resultados de uma simulação, com 1000 réplicas,

de um modelo ARFIMA(0,d,1).

\begin{tabular}{|c|c|c|c|c|c|}
\hline \multirow{2}{*}{$\begin{array}{l}\mathrm{T}=12 \mathrm{~S} \\
\text { Estimador }\end{array}$} & \multicolumn{2}{|c|}{$\mathrm{d}=0.3$} & \multirow[b]{2}{*}{ Estimador } & \multicolumn{2}{|c|}{ Thet $a=-0.6$} \\
\hline & $\hat{\operatorname{lar}(\hat{d})}$ & $\hat{B}(\hat{d})$ & & $\operatorname{Var}\left(\hat{\theta}_{1}\right)$ & $\hat{B}\left(\hat{\theta}_{1}\right)$ \\
\hline$\hat{d}_{c}$ & 0.002 & -0.012 & $\hat{\theta}_{c}$ & 0.004 & 0.088 \\
\hline$\hat{d}_{c g}$ & 0.002 & -0.015 & $\hat{\theta}_{c g}$ & 0.004 & 0.082 \\
\hline$\hat{d}_{p}$ & 0.072 & 0.013 & $\hat{\theta}_{p}$ & 0.011 & 0.081 \\
\hline$\hat{d}_{p s}$ & 0.041 & -0.064 & $\hat{\theta}_{p s}$ & 0.009 & 0.061 \\
\hline$\hat{d}_{m c}$ & $0.00 \tau$ & -0.056 & $\hat{\theta}_{m e}$ & 0.006 & -0.022 \\
\hline$\hat{d}_{n}$ & 0.019 & $-0.0: 31$ & $\hat{\theta}_{u}$ & 0.006 & 0.072 \\
\hline $\mathrm{T}=256$ & \multicolumn{2}{|c|}{$\mathrm{d}=0.3$} & & \multicolumn{2}{|c|}{ Theta $=-0.6$} \\
\hline Estimador & $\hat{\operatorname{Var}}(\hat{d})$ & $\hat{B}(\hat{d})$ & Estimador & $\hat{V a r}\left(\hat{\theta}_{1}\right)$ & $\hat{B}\left(\hat{\theta}_{1}\right)$ \\
\hline$\hat{d}_{c}$ & 0,001 & 0,008 & $\hat{\theta}_{c}$ & 0,002 & 0,094 \\
\hline$\hat{d}_{c g}$ & 0.001 & 0,000 & $\hat{\theta}_{c g}$ & 0.002 & 0.091 \\
\hline$\hat{d}_{p}$ & 0.046 & 0.024 & $\hat{\theta}_{p}$ & $0.00 \tau$ & 0,087 \\
\hline$\hat{d}_{p s}$ & 0.026 & -0.051 & $\hat{\theta}_{p s}$ & 0.005 & 0.067 \\
\hline$\hat{d}_{m v}$ & $0.00: 3$ & $-0.0: 31$ & $\hat{\theta}_{m v}$ & 0.003 & -0.014 \\
\hline$\hat{d}_{w}$ & 0.016 & -0.050 & $\hat{\theta}_{u}$ & 0,004 & 0,068 \\
\hline $\mathrm{T}=512$ & \multicolumn{2}{|c|}{$\mathrm{d}=0.3$} & & \multicolumn{2}{|c|}{ Thet $\mathrm{a}=-0.6$} \\
\hline Estimador & $\hat{\operatorname{Var}(\hat{d})}$ & $\hat{B}(\hat{d})$ & Estimador & $\operatorname{Var}\left(\hat{\theta}_{1}\right)$ & $\hat{B}\left(\hat{\theta}_{1}\right)$ \\
\hline$\hat{d}_{c}$ & 0.001 & 0.018 & $\hat{\theta}_{c}$ & 0.001 & 0,101 \\
\hline$\hat{d}_{c y}$ & 0,001 & 0,012 & $\hat{\theta}_{c g}$ & 0,001 & 0,098 \\
\hline$\hat{d}_{p}$ & 0.029 & -0.001 & $\hat{\theta}_{p}$ & 0.005 & $0,08: 3$ \\
\hline$\hat{d}_{p s}$ & 0,017 & $-0,0: 36$ & $\hat{\theta}_{p s}$ & 0.009 & 0.075 \\
\hline$\hat{l}_{m v}$ & 0.002 & $-0,016$ & $\hat{\theta}_{m u}$ & 0.002 & $-0.00 \tau$ \\
\hline$\hat{d}_{w}$ & 0.016 & $-0,049$ & $\hat{\theta}_{u}$ & 0.004 & 0.069 \\
\hline
\end{tabular}




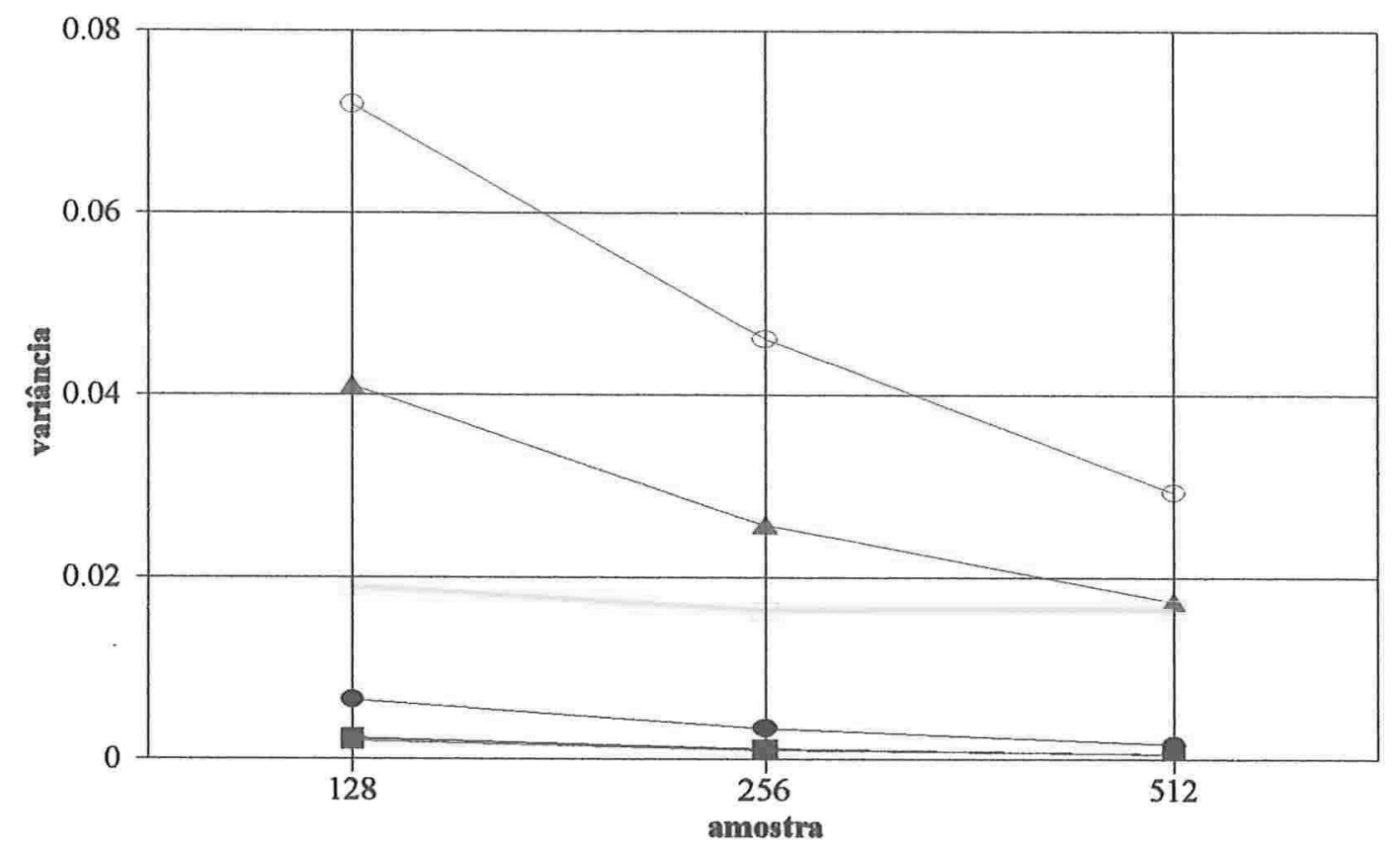

$\longrightarrow \hat{d}_{c} \rightarrow \hat{d}_{c g}-\odot \hat{d}_{p} \rightarrow \hat{d}_{p s} \rightarrow \hat{d}_{m v} \rightarrow \hat{d}_{w}$

Figura 6.8: Variância estimada dos estimadores de $d$. 


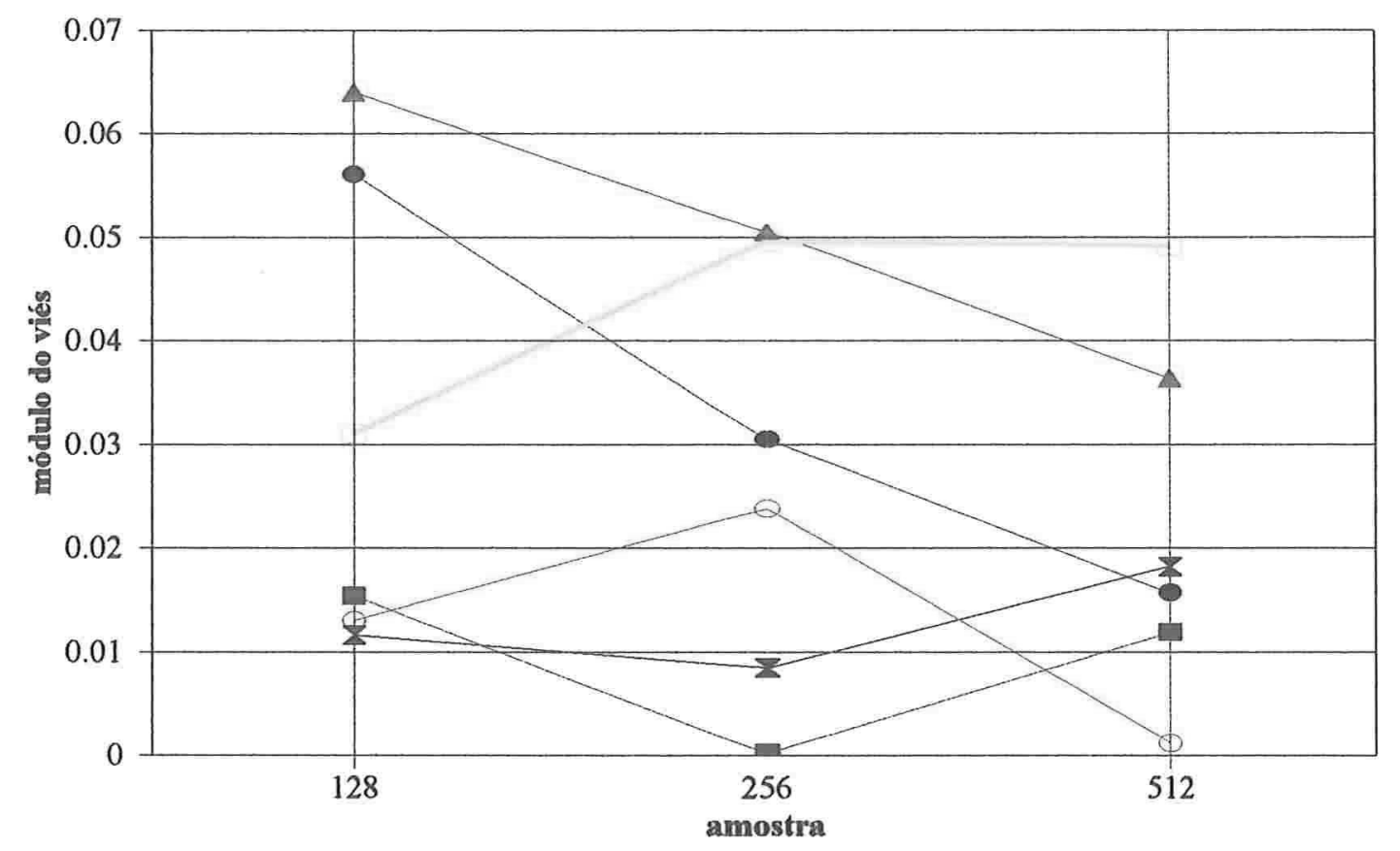

$\rightarrow \quad \hat{d}_{c} \rightarrow-\hat{d}_{c g}-\ominus \hat{d}_{p} \rightarrow \hat{d}_{p s} \rightarrow \hat{d}_{m v} \longrightarrow \hat{d}_{w}$

Figura 6.9: Módulo do viés estimado dos estimadores de $d$. 
A Figura 6.9 apresenta o gráfico do riés estimado dos estimadores de $d$ para $T=128.256$ e jl2. Segundo este gráfico. podemos dizer que:

1. O comportamento do viés. à medida que aumentamos o tamanho da amostra. varia de estimador para estimador. No caso dos estimadores $\hat{d}_{c}$, $\hat{d}_{c g}$ e $\hat{d}_{p}$, não existe um comportamento do viés estimado que indique alguma tendência em relaçào ao tamanho da amostra. Os estimadores $\hat{d}_{p s}$ e $\hat{d}_{m v}$ apresentam $u$ comportamento do viés que indica uma tendência de decrescimento deste quando $T \rightarrow \infty$. O estimador $\hat{d}_{w}$ é o único que apresenta uma tendência de crescimento do viés quando aumentamos o tamanho da amostra.

2. A classificaçào dos estimadores com base no viés estimado nào é constante para todos os tamanhos de amostra. ou seja. para cada tamanho de amostra utilizado a classificaçào muda.

3. Em geral, temos os estimadores $\hat{d}_{c}, \hat{d}_{c y}$ e $\hat{d}_{p}$ como os menos viesados, enquanto $\hat{d}_{p s}$ e $\hat{d}_{w}$ sào os mais viesados. O estimador $\hat{d}_{m v}$ apresenta um decaimento muito rápido do viés com o aumento do tamanho da amostra e. por isso, passa de um dos estimadores mais viesados. quando $\mathrm{T}=12 \mathrm{~S}$. para o segundo menos viesado. quando $\mathrm{T}=512$.

A Figura 6.10 apresenta a variância estimada dos estimadores de $\theta_{1}$ para os três tamanhos de amostra. Com base neste gráfico, temos que:

1. As estimativas das variâncias do todos os estimadores de $\theta_{1}$ diminuem quando aumentamos o tamanho da amostra.

2. Os estimadores $\hat{\theta}_{c}$ e $\hat{\theta}_{c y}$ apresentam as menores rariàncias estimadas, enquanto $\hat{\theta}_{m v}$ aparece com a segunda menor estimativa da variância. para todos os tamanhos de amostra.

3. O estimador $\hat{\theta}_{p}$ possui a maior estimativa de variância para todos os tamanhos de amostra.

A Figura 6.11 apresenta o gráfico das estimativas do viés dos estimadores de $\theta_{1}$. Com base neste gráfico. temos que: 


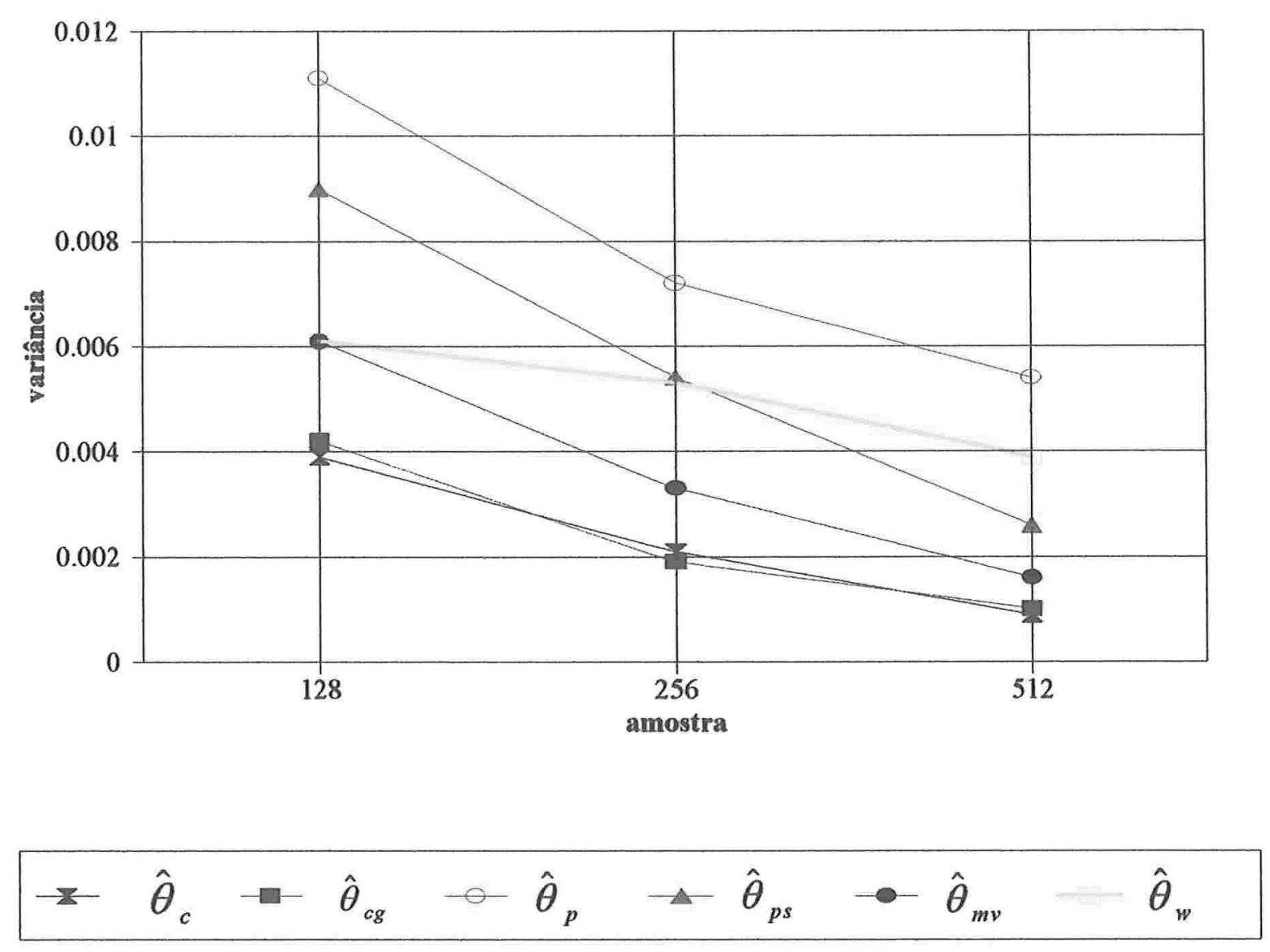

Figura 6.10: Variância estimada dos estimadores de $\theta_{1}$. 


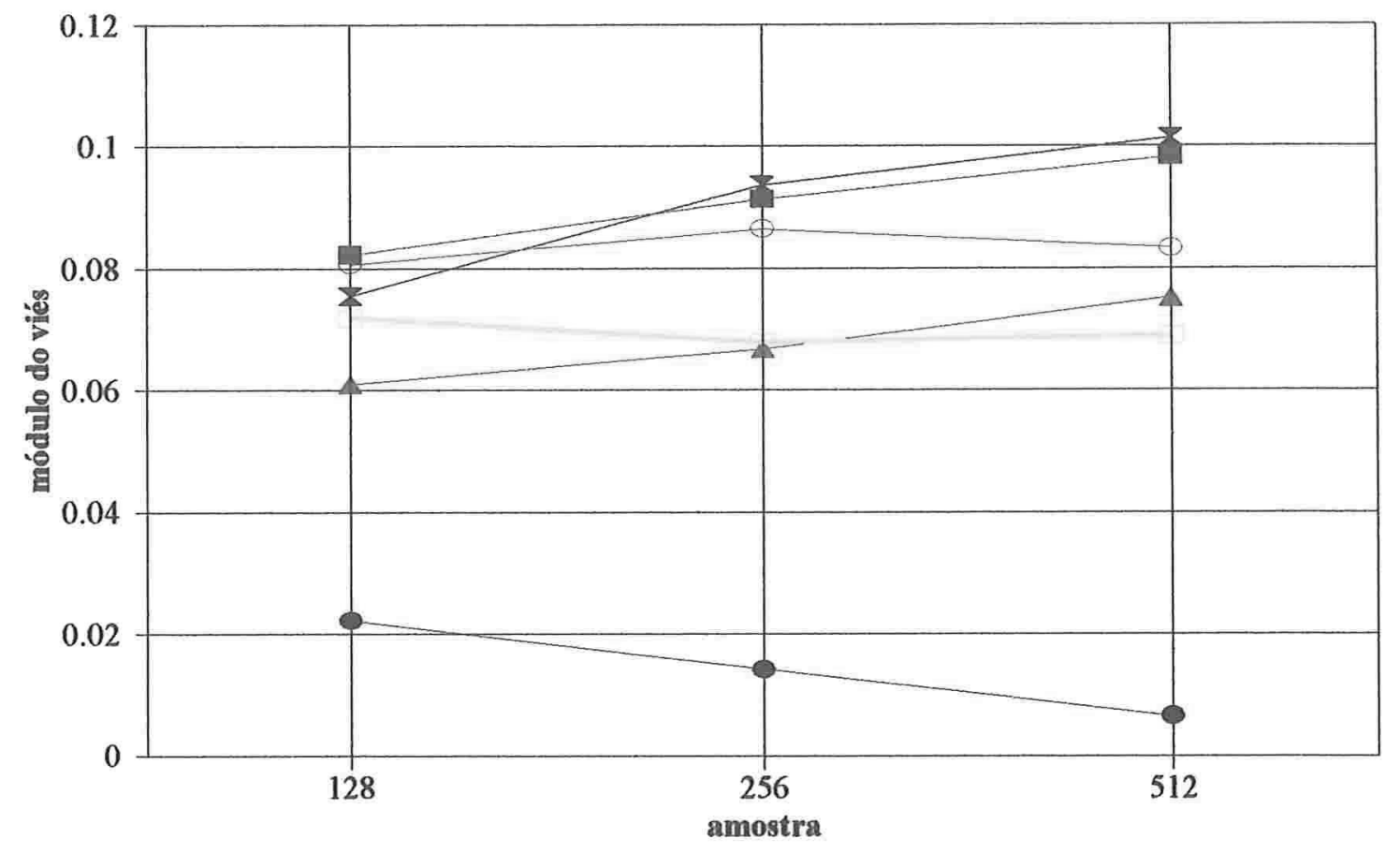

$\longrightarrow \hat{\theta}_{c} \rightarrow \hat{\theta}_{c g} \multimap \hat{\theta}_{p} \rightarrow \hat{\theta}_{p s} \rightarrow \hat{\theta}_{m v} \longrightarrow \hat{\theta}_{w}$

Figura 6.11: Módulo do viés estimado dos estimadores de $\theta_{1}$. 
1. Com o aumento da amostra as estimativas do viés dos estimadores $\hat{\theta}_{m v}$ e $\hat{\theta}_{w}$ diminuem. enquanto os estimativas do viés de $\hat{\theta}_{.} . \hat{\theta}_{g}$ e $\hat{\theta}_{p s}$ aumentam. As estimativas do viés de $\hat{\theta}_{p}$ nào apresentam alguma tendência em relaçào ao tamanho da amostra.

2. As estimativas do viés de $\hat{\theta}_{m v}$ sào bem menores que as dos demais estimadores, para todos os tamanhos de amostra.

3. Para $\mathrm{T}=128$. o estimador $\hat{\theta}_{p s}$ aparece como o segundo menos viesado e $\hat{\theta}_{c}, \hat{\theta}_{c g}, \hat{\theta}_{p}$ e $\hat{\theta}_{w}$ possuem estimativas do viés muito próximas, além destes estimadores serem os mais viesados.

4. Para $\mathrm{T}=2.56 . \hat{\theta}_{p s}$ e $\hat{\theta}_{w}$ sào os estimadores que apresentam as segundas menores estimativas do viés e $\hat{\theta}_{p s} . \hat{\theta}_{e}$ e $\hat{\theta}_{\varepsilon}$ sào os estimadores mais viesados.

5. Para $\mathrm{T}=512$. os estimadores $\hat{\theta}_{w}, \hat{\theta}_{p s}$ e $\hat{\theta}_{p}$ apresentam as segundas menores estimativas do viés. enquanto $\hat{\theta}_{\varepsilon}$ e $\hat{\theta}_{c g}$ sào os mais viesados.

Resumindo as informaçòes obtidas com os quatro gráficos analisados anteriormente. temos o seguinte:

- Os estimadores $\hat{d}_{\varepsilon}$ e $\hat{d}_{c g}$ apresentaram estimativas da variância e do viés estimados abaixo das dos demais estimadores de $d ; \hat{\theta}_{c} e \hat{\theta}_{c g}$ também apresentaram estimativas das variâncias pequenas. mas as estimativas lo viés desses estimadores foram as maiores.

- O estimador $\hat{d}_{p}$ apresentou a maior variância estimada. entretanto, seu viés estimado foi o menor: o estimador $\hat{\theta}_{p}$ foi um dos mais viesados e com a maior variância estimada.

- O estimador $\hat{d}_{p s}$ se apresentou como $u m$ dos mais viesados e suas estimativas de variância ficou entre as maiores; $\hat{\theta}_{p s}$, em geral, apresentou valores intermediários para as estimativas do viés e da variância.

- O estimador $\hat{d}_{m x}$. em relaçào ao viés. porle ser considerado um estimador intermediário. mas com relaçào à variância. é un dos melhores estimadores : $\hat{\theta}_{m v}$ também apresentou boas estimativas da variância e do viés. 
- $\hat{d}_{u}$ é um dos estimadores mais viesados. mas obteve bons resultados com relaçào à variância estimada: $\hat{\theta}_{u}$ se classificou. segundo o viés. como um estimador intermediário. entretanto, este estimador nào possui boas estimativas de variância. sendo classificado, segundo a variância. como um dos piores estimadores.

A seguir apresentamos a Tabela 6.6 com a classificaçào pelo erro quadrático médio estimado $(E \hat{Q} . M)$ dos estimadores dos parâmetros $d$ e $\theta_{1}$. para todos os tamanhos de amostra. Com base nesses resultados. temos que:

1. A classificaçào dos estimadores de $d$. em relaçào ao EQQ.M. nào se modifica com o aumento do tamanho da amostra. Entretanto. as diferenças entre os $E \hat{Q} M^{\prime}$ 's desses estimadores diminui muito. ('om isso. para $\mathrm{T}=12 \mathrm{~S}$. $\hat{d}_{c}$ e $\hat{d}_{c \mathrm{~g}}$ são os melhores estimadores de d. $\hat{d}_{m u}$ é o segundo melhor estimador. $\hat{d}_{w}$ é o terceiro. $\hat{d}_{p s}$ e $\hat{d}_{p}$ sào os piores. Porém. para $\mathrm{T}=512 . \hat{d}_{c} \cdot \hat{d}_{\varepsilon y}$ e $\hat{d}_{m v}$ podem ser considerados os melhores estimadores de $d, \hat{d}_{p s}$ e $\hat{d}_{w}$ pertencem ao grupo que se classifica en segundo lugar e $\hat{d}_{p}$ continua sendo o pior estimador do parâmetro, segundo o $E \hat{Q} M$.

2. A classificação dos estimadores de $\theta_{1}$, muda com o aumento do tamanho da amostra, mas os valores dos $E \hat{Q} . Y^{\prime}$ s dos estimadores $\hat{\theta}_{u} . \hat{\theta}_{c g}, \hat{\theta}_{c}$ e $\hat{\theta}_{p s}$ são muito próximos para todos os tamanhos de amostra. Com isso. esses estimadores formam um grupo dos estimadores classificados em segundo lugar. () melhor estimador, com base no EQ̂M. é $\hat{\theta}_{m v}$ e o pior estimador é $\hat{\theta}_{p}$.

Com base nessas análises podemos dizer que:

- Os métodos da autocorrelaçào. da autocorrelação generalizado e de máxima verossimilhança podem ser considerados os melhores métodos de estimaçào dos parâmetros do modelo ARFI.MA(0.d,1).

- Os métodos das ondaletas e do periodograma suavizado podem ser classificados como métodos intermediários.

- O método do periodograma se mostros o pior método de estimaçào dos parâmetros do modelo ARFIXIA(0.d.1). 
Tabela 6.6: Classificação dos estimadores de $d$ e $\theta_{1}$ pelo $E \hat{Q} . M$

\begin{tabular}{|l|c|}
\hline $\mathrm{T}=128$ & $\mathrm{~d}=0.3$ \\
\cline { 2 - 2 } Estimador & $E \hat{Q} . M(\hat{d})$ \\
\hline$\hat{d}_{c g}$ & 0.002 \\
$\hat{d}_{c}$ & 0.002 \\
$\hat{d}_{m v}$ & 0.010 \\
$\hat{d}_{w}$ & 0.020 \\
$\hat{d}_{p s}$ & 0.046 \\
$\hat{d}_{p}$ & 0.072 \\
\hline \hline $\mathrm{T}=256$ & $\mathrm{~d}=0.3$ \\
\cline { 2 - 2 } Estimador & $E \hat{Q} . M(\hat{d})$ \\
\hline$\hat{d}_{c g}$ & 0.001 \\
$\hat{d}_{c}$ & 0.001 \\
$\hat{d}_{m v}$ & 0.004 \\
$\hat{d}_{w}$ & 0.019 \\
$\hat{d}_{p s}$ & 0.028 \\
$\hat{d}_{p}$ & 0.047 \\
\hline \hline $\mathrm{T}=512$ & $\mathrm{~d}=0.3$ \\
\cline { 2 - 2 } Estimador & $E \hat{Q} M(\hat{d})$ \\
\hline$\hat{d}_{c g}$ & 0.001 \\
$\hat{d}_{c}$ & 0.001 \\
$\hat{d}_{m v}$ & 0.002 \\
$\hat{d}_{w}$ & 0.019 \\
$\hat{d}_{p s}$ & 0.019 \\
$\hat{d}_{p}$ & 0.029 \\
\hline
\end{tabular}

\begin{tabular}{|c|c|}
\hline \multirow{2}{*}{$\begin{array}{l}\mathrm{T}=128 \\
\text { Estimador }\end{array}$} & $\theta_{1}=-0.6$ \\
\hline & $E \hat{Q} . M\left(\hat{\theta}_{1}\right)$ \\
\hline$\hat{\theta}_{m v}$ & 0.007 \\
\hline$\hat{\theta}_{c y}$ & 0.011 \\
\hline$\hat{\theta}_{w}$ & 0.012 \\
\hline$\hat{\theta}_{c}$ & 0.012 \\
\hline$\hat{\theta}_{p s}$ & 0.012 \\
\hline$\hat{\theta}_{p}$ & 0.018 \\
\hline $\mathrm{T}=256$ & $\theta_{1}=-0.6$ \\
\hline Estimador & $E \hat{Q} . M\left(\hat{\theta}_{1}\right)$ \\
\hline$\hat{\theta}_{m v}$ & 0.004 \\
\hline$\hat{\theta}_{w}$ & 0.009 \\
\hline$\hat{\theta}_{p s}$ & 0.010 \\
\hline$\hat{\theta}_{c g}$ & 0.010 \\
\hline$\hat{\theta}_{c y}$ & 0.011 \\
\hline$\hat{\theta}_{p}$ & 0.015 \\
\hline $\mathrm{T}=512$ & $\theta_{1}=-0.6$ \\
\hline Estimador & $E \hat{Q} \cdot M\left(\hat{\theta}_{1}\right)$ \\
\hline$\hat{\theta}_{m v}$ & 0.002 \\
\hline$\hat{\theta}_{w}$ & 0.009 \\
\hline$\hat{\theta}_{p s}$ & 0.009 \\
\hline$\hat{\theta}_{c g}$ & 0.011 \\
\hline$\hat{\theta}_{c}$ & 0.011 \\
\hline$\hat{\theta}_{p}$ & 0.012 \\
\hline
\end{tabular}




\section{Capítulo 7}

\section{Aplicação a uma série real}

\subsection{Série de Vazão do Rio São Marcos}

Neste capítulo ajustaremos o modelo ARFIMA(p,d.q) a uma série de vazão do Rio Sào Marcos, ver Apêndice C'. (fonte: C'ESP - Companhia Furnas) utilizando todos os métodos de estimaçào do parâmetro de longa memória, $d$. estudados no Capítulo 5. Além disso, ajustaremos os modelos auto-regressivo $(A R(p))$ e $S . A R I M A(p . d . q) \times(P . D . Q)_{12}$, com o objetivo de comparar os modelos $A R F I M A(p . d . q)$ a esses modelos mais tradicionais. No caso dos modelos $A R(p)$. sabemos que estes eram indicados para modelar séries que apresentavam comportamento de longa memória antes do aparecimento dos modelos ARFIMA(p,d,q), entretanto, o excesso de parâmetros poderia prejudicar a previsào.

A série hidrológica de vazào do Rio São Marcos é apresentada na Figura 7.1,onde podemos perceber que a variância desta série nào é constante em relação ao tempo, podemos ver também que as últimas observaçòes desta série possuem uma variaçào maior que as demais. A necessidade de uma transformaçào pode ser constatada através da Figura 7.2 , onde as 540 observaçòes da série foram divididas em 45 subséries de 12 elementos cada e foram calculadas as médias e variaçòes de amplitude de cada segmento.

Com base neste gráfico, podemos constatar o aumento da variaçào dos dados conforme a média das subséries aumenta. Sendo assim, aplicamos o logarítmo na série de vazão com o objetivo de melhorar esse comportamento. O gráfico da série transformada, bem como 


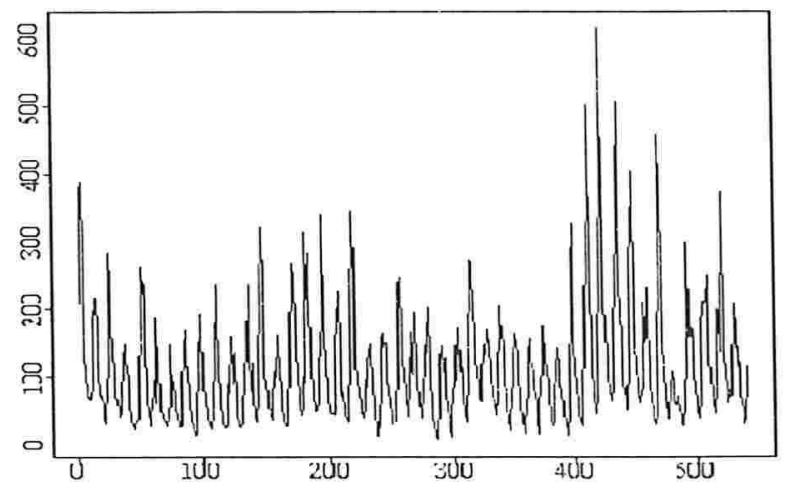

Figura T.1: Série de vazão do Rio Sào Marcos - ('ESP

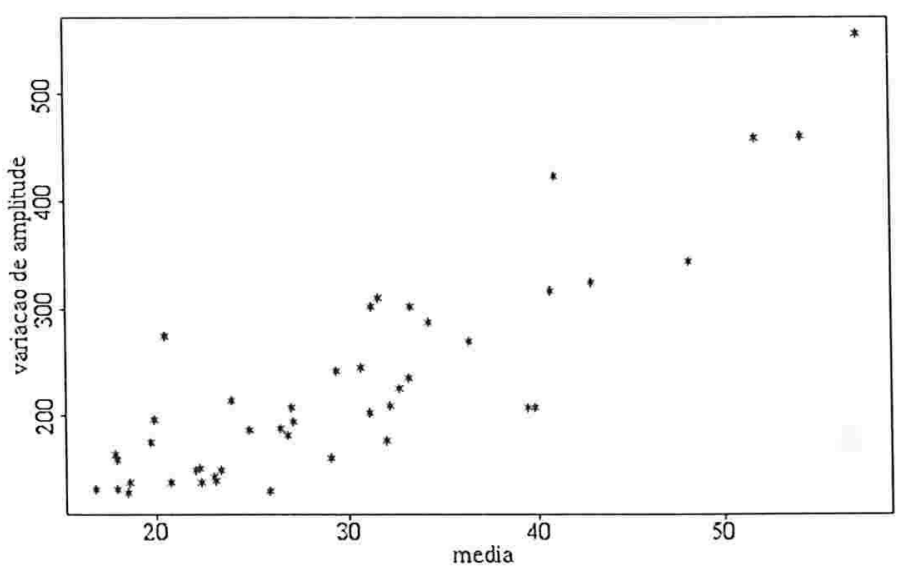

Figura 7.2 : Gráfico da média $\mathrm{x}$ variação de amplitude das 4.5 subséries 
suas funçòes de autocorrelaçào e autocorrelação parcial amostrais e o seu periodograma são apresentados na Figura 7.3. A adequaçào da aplicaçào do logarítmo nos dados pode ser confirmada pelo gráfico da série transformada. no qual o comportamento verificado na série original nào se apresenta. O gráfico da função de autocorrelação amostral apresenta um evidente comportamento de longa memória da série de vazão, ou seja, mostra a forte correlaçào entre observaçòes da série distanciadas por um grande intervalo no tempo, este gráfico também mostra o forte comportamento sazonal da série. A partir da série transformada, iremos ajustar os modelos já citados no início deste capítulo.
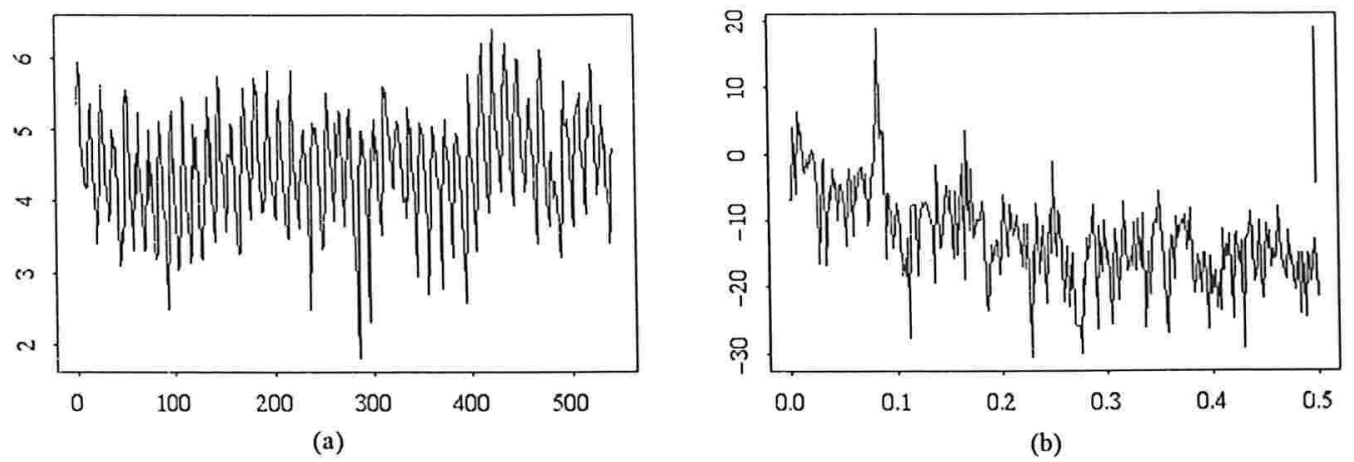

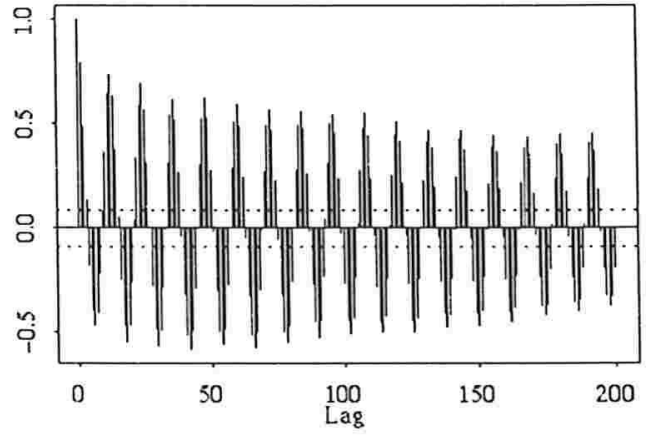

(c)

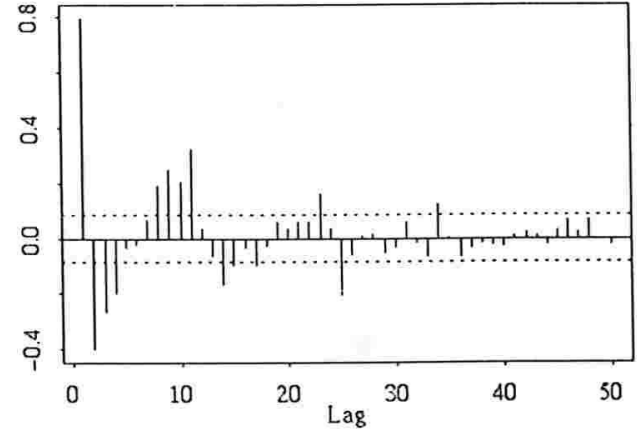

(d)

Figura 7.3: (a) Série transformada. (b) Periodograma, (c) Função de autocorrelação amostral e (d)Funçào de autocorrelaçào parcial amostral 


\subsubsection{Ajustamento dos modelos}

a) Modelo Auto-regressivo $(\mathrm{AR}(\mathrm{p}))$.

Para a série de vazào, ajustamos um modelo auto-regressivo de ordem $25(\operatorname{AR}(25))$. As estimativas dos parâmetros e seus desvios padrões sào apresentados na Tabela T.1.

Tabela 7.1 - Resultados para o ajuste do modelo $\mathrm{AR}(25)$ à Série de Vazão

\begin{tabular}{|c|c|c|c|}
\hline Parâmetro & Estimativas & Desvio Padrão & T-valor \\
\hline$\hat{O}_{1}$ & 0.7128 & $0.0+22$ & 16.89 \\
\hline$\hat{\phi}_{2}$ & $-0.032 t$ & 0.0516 & -0.62 \\
\hline$\hat{O}_{3}$ & $0.00: 31$ & 0.051 .4 & 0.06 \\
\hline$\hat{\phi}_{4}$ & -0.0536 & $0.0 \div 1: 3$ & -1.04 \\
\hline$\hat{O}_{5}$ & 0.0800 & $0.051: 3$ & 1.56 \\
\hline$\hat{O}_{6}$ & $0.029 \bar{\top}$ & $0.051+4$ & 0.58 \\
\hline$\hat{\phi}_{\tau}$ & -0.0630 & 0.0514 & -1.23 \\
\hline$\hat{\phi}_{8}$ & -0.0328 & 0.0513 & -0.64 \\
\hline$\hat{\phi}_{9}$ & 0.1003 & 0.0513 & 1.95 \\
\hline$\hat{O}_{10}$ & -0.1201 & 0.0514 & -2.34 \\
\hline$\hat{\phi}_{11}$ & $0.2 \cdot 2 \cdot 29$ & 0.0515 &,+ 33 \\
\hline$\hat{o}_{12}$ & $0.0+7 \overline{1}$ & 0.0523 & 0.91 \\
\hline$\hat{\phi}_{13}$ & 0,0646 & 0.0523 & $1.2: 3$ \\
\hline$\hat{\phi}_{14}$ & $-0.0+78$ & $0.052: 3$ & -0.91 \\
\hline$\hat{\phi}_{15}$ & -0.0951 & 0.0515 & -1.85 \\
\hline$\hat{\phi}_{16}$ & $0.056-$ & $0,051 \cdot 4$ & 1.10 \\
\hline$\hat{O}_{17}$ & $-0.0 \bar{\tau}+3$ & 0.0513 & -1.45 \\
\hline$\hat{O}_{18}$ & -0.0894 & 0.0513 & $-1 . \overline{1 . t}$ \\
\hline$\hat{\phi}_{19}$ & 0.0480 & 0.051 .4 & 0.93 \\
\hline$\hat{\phi}_{20}$ & 0.0130 & 0.0514 & 0.25 \\
\hline$\hat{O}_{21}$ & $0.01+1$ & 0.0513 & $0.2 \bar{\imath}$ \\
\hline$\hat{\phi}_{22}$ & $-0,0521$ & 0.0513 & -1.02 \\
\hline$\phi_{23}$ & 0,1239 & 0.0513 & 2.41 \\
\hline$\hat{\phi}_{24}$ & 0.17 .56 & 0.0516 & 3.40 \\
\hline$\hat{O}_{25}$ & $-0.201+$ & $0.0 \cdot+2 \cdot 2$ & $-4 . \bar{i}$ \\
\hline$\hat{\sigma}_{a}^{2}$ & 0.1033 & & \\
\hline
\end{tabular}


Pela Tabela 7.1 . apenas as estimativas dos parâmetros $O_{1} \cdot O_{9} \cdot O_{11} \cdot O_{23}, O_{24}$ e $O_{25}$ sào significativamente diferentes de zero ao nivel de significancia de 0.0\%). Entretanto. os gráficos das funçòes de autocorrelaçào e autocorrelaçào parcial residuais. dados na Figura 7.4. não apresentam nenhum valor significativamente diferente de zero. o que indica um ótimo comportamento dos resíduos.

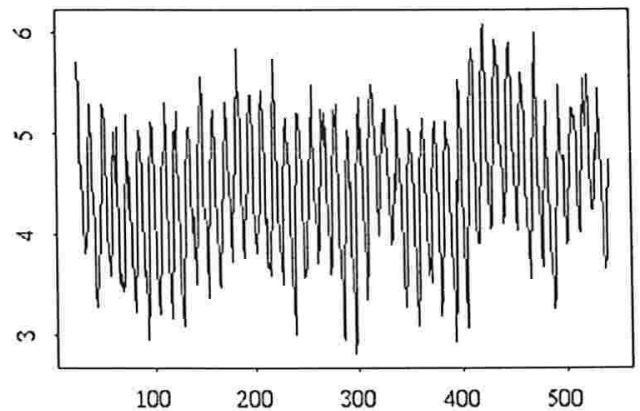

(a)

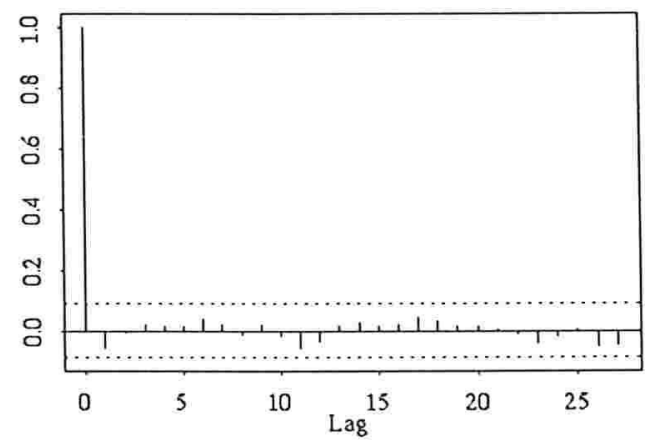

(c)

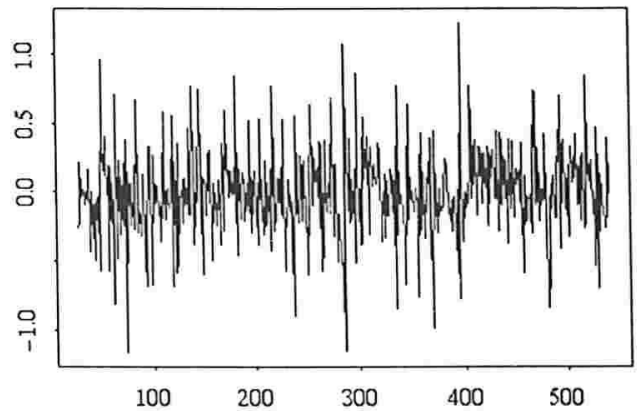

(b)

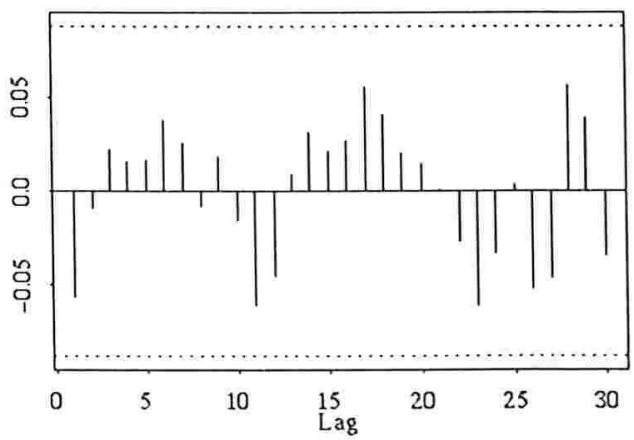

(d)

Figura 7.4: (a) Série ajustada, (b) Resíduos, (c) Funçào de autocorrelação amostral residual e (d) Funçào de autocorrelaçào parcial amostral residual.

b) Modelo S.ARI.YA(p.d.q) $\times(P . D . Q)_{12}$

No caso deste modelo. inicialmente ajustamos o modelo S. $A R I . M A(1,0,0) \times(0,1.1)_{12}$, cujos resultados da estimaçào dos parâmetros são apresentados na Tabela 7.2 . 
Tabela 7.2 - Resultados do ajuste do modelo S.ARI.M.A(1.0.0) × (0.1.1) 12 à Série de Vazão

\begin{tabular}{|l|r|r|r|}
\hline Parâmetro & Estimativa & Desvio Padrào & T-valor \\
\hline$\hat{o}_{1}$ & 0.6859 & $0.031 \bar{\imath}$ & 21.64 \\
$\hat{\theta}_{12}$ & 0.9279 & 0.0162 & 52.28 \\
\hline \hline$\hat{\sigma}_{n}^{2}$ & 0.1032 & & \\
\hline
\end{tabular}

Com base nesta tabela. podemos ver que as estimativas dos parâmetros são significativamente diferentes de zero. entretanto. os gráficos das funções de autocorrelação e autocorrelaçào parcial amostrais dos resíduos. dados na Figura 7.j). mostram que. para o "lag" 6. essas funçòes apresentam valores altos. Também podemos perceber que a funçào de autocorrelaçào parcial apresenta valores significativamente diferentes de zero para os "lags" 12 e 24. Com isso, vamos inserir os parâmetros auto-regressivos de ordem 6 e 12 ajustando o modelo S.ARIMA(6.0.0) $\times(1.1 .1)_{12}$, onde $O_{2}=\varphi_{3}=O_{4}=o_{5}=0$.

Os resultados das estimativas dos parâmetros desse modelo e seus respectivos desvios padrões sào dados na Tabela 7.3 , onde todas as estimativas dos parâmetros do modelo são significativamente diferentes de zero ao nível de significância de 0.05.

Tabela 7.3 - Resultados do ajuste do modelo

$$
\text { S.ARI.MA(6.0.0) } \times(1,1.1)_{12} \text { à Série de Vazão }
$$

\begin{tabular}{|c|c|c|c|}
\hline Parâmetro & Estimativa & Desvio Padrào & P-valor \\
\hline$\phi_{1}$ & $0,6+47$ & 0.0 .138 & 1.4 .72 \\
\hline$\hat{O}_{6}$ & 0.143 .4 & 0.0 .417 & 3.21 \\
\hline$\dot{\phi}_{12}$ & -0.1228 & 0.0501 & $2 .+1$ \\
\hline$\hat{\theta}_{12}$ & $0,8+70$ & 0.0287 & 2.95 \\
\hline$\hat{\sigma}_{a}^{2}$ & i & & \\
\hline
\end{tabular}

Os gráficos do ajuste. e das funçòes de autocorrelaçào e autocorrelação parcial são apresentados no Figura 7.6 , onde percebemos que a inserçào dos parâmetros $\oint_{12}$ e $\phi_{6}$ melhoraram o a juste da série. pois as funções de autocorrelação amostral e de autocorrelação parcial amostral dos resíduos indicam um bom comporatamento dos resíduos. ou seja. nào apresentam valores que justifiquem a inadequaçào do modelo ajustado. 

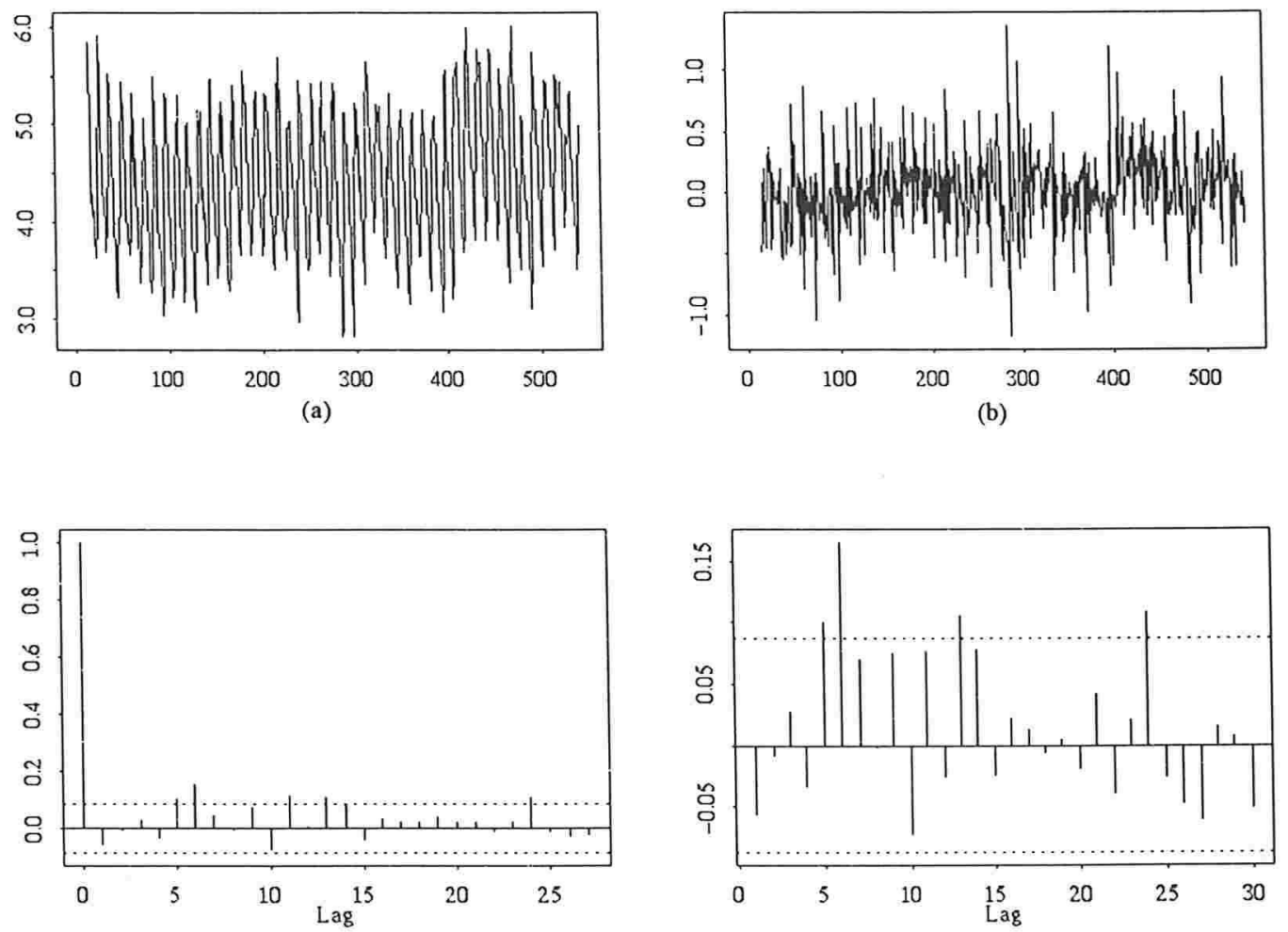

(c)

(d)

Figura 7.5: (a) Série ajustada, (b) Resíduos. (c) Função de autocorrelação amostral e (d) Funçào de autocorrelaçào parcial amostral. 

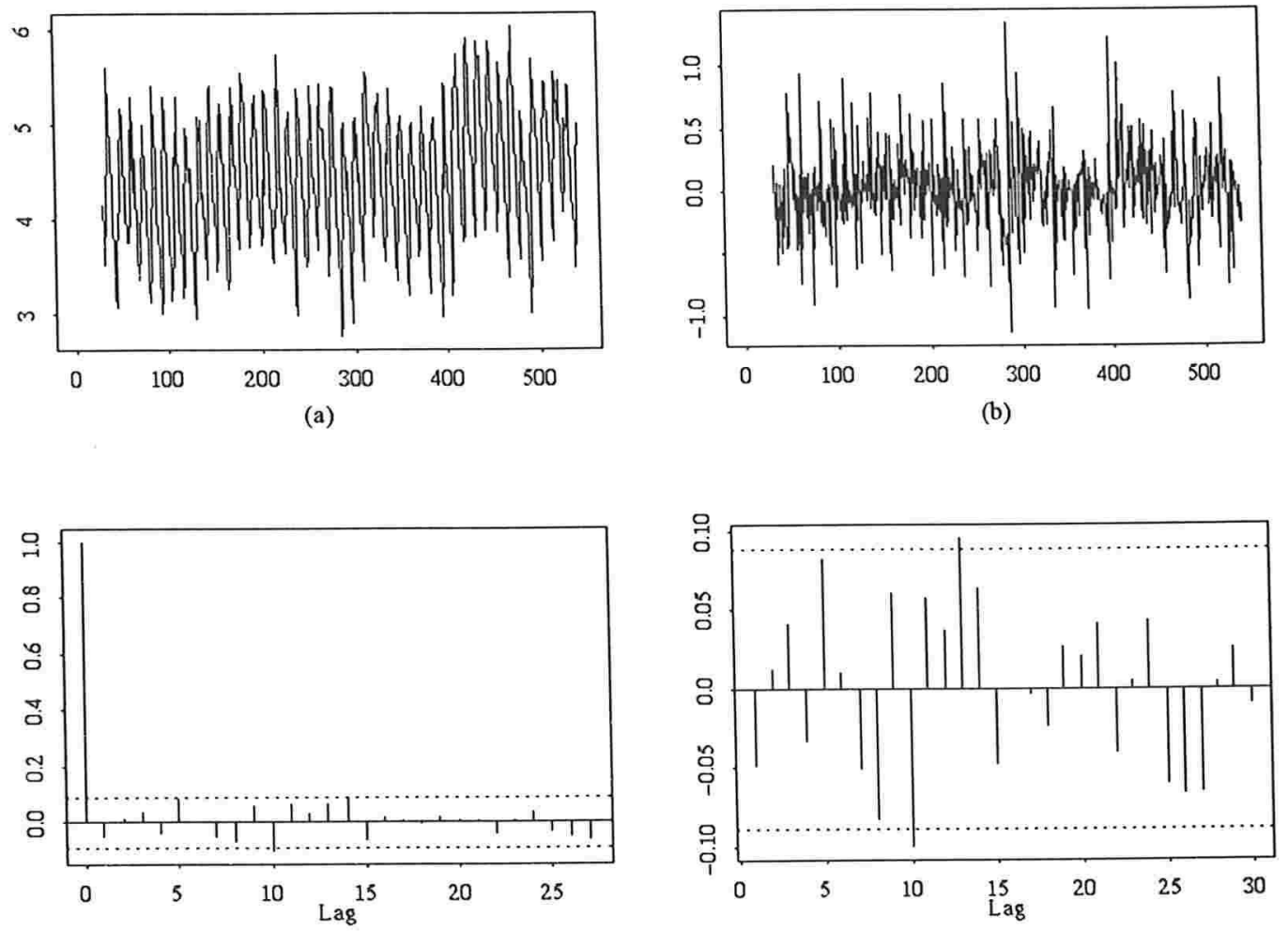

(c)

(d)

Figura 7.6: (a) Série ajustada, (b) Resíduos. (c) Função de autocorrelação amostral e (d) Função de autocorrelação parcial amostral. 
c) Modelo ARFIMA $(p, d, q)$

A princípio, ajustamos o modelo $A R F I M A(0, d .0)$ para todos os seis métodos de estimaçào do parâmetro $d$. Os gráficos da função de autocorrelaçào amostral dos resíduos desse ajuste, para os seis estimadores de $d$, são apresentados na Figura 7.7. Da mesma forma, temos os gráficos da função de autocorrelação parcial amostral na Figura 7.8.

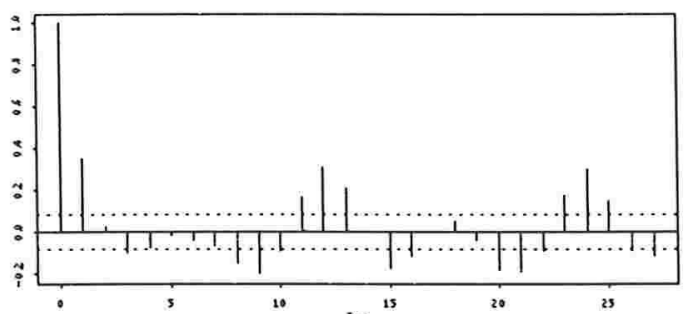

(a)

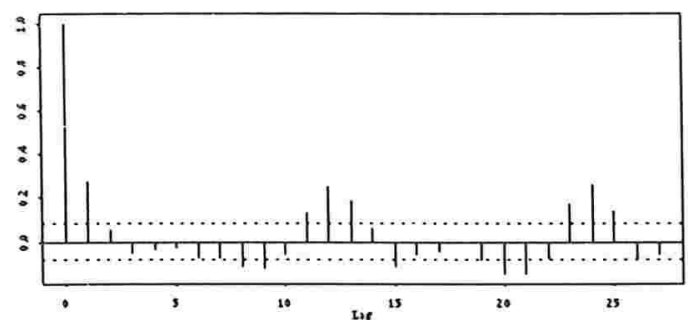

(c)

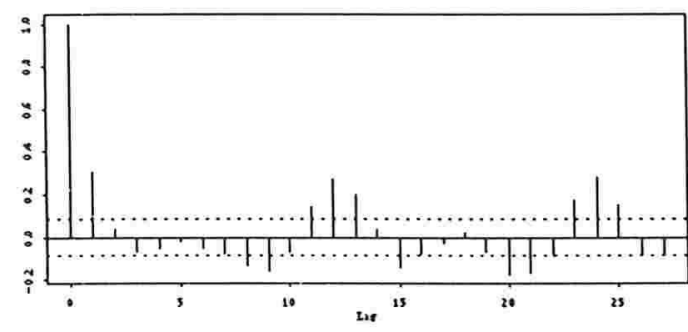

(e)

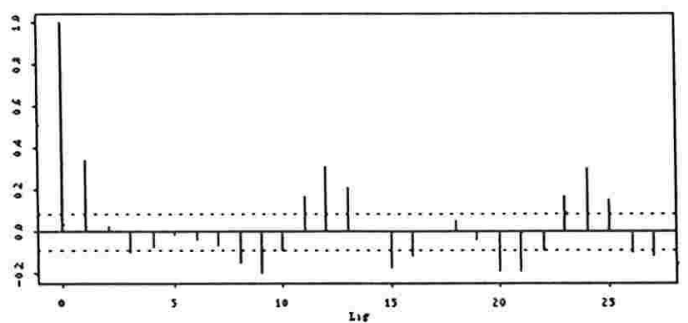

(b)

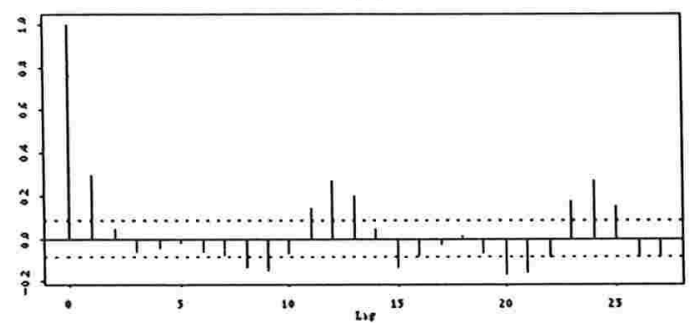

(d)

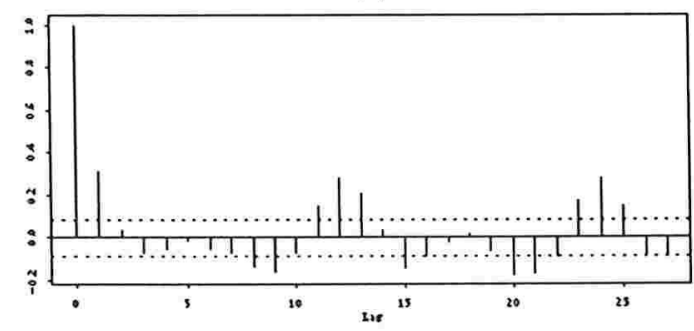

(f)

Figura 7.7: Função de autocorrelação amostral dos resíduos: (a) estimador $\hat{d}_{c}$, (b) estimador $\hat{d}_{c g},(\mathrm{c})$ estimador $\hat{d}_{p},(\mathrm{~d})$ estimador $\hat{d}_{p s},(\mathrm{e})$ estimador $\hat{d}_{m v},(\mathrm{f})$ estimador $\hat{d}_{w}$.

Podemos perceber pelas Figuras 7.7 e 7.8 que o comportamento sazonal permanece nos resíduos do modelo $A R F I M A(0, d, 0)$, além disso esse comportamento é bastante parecido para todos os seis métodos de estinação de $d$. Com base nesses gráficos, ajustaremos o modelo $S A R F I M A(1, d, 0) \times(0,1,1)_{12}$ à Série de Vazão, onde serão utilizados os seis estimadores de $d$. Desta forma, teremos seis modelos $S . A R F I M A(1, d, 0) \times(0,1,1)_{12}$ ajustados. 


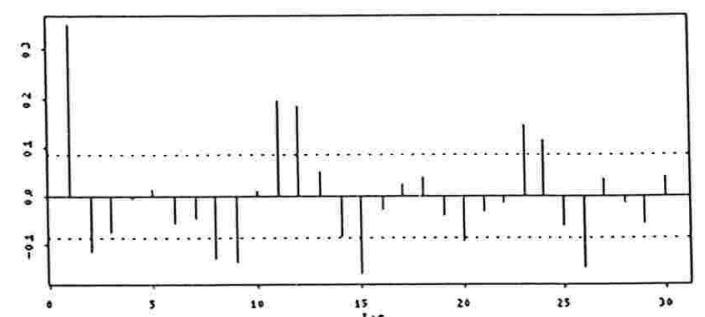

(a)

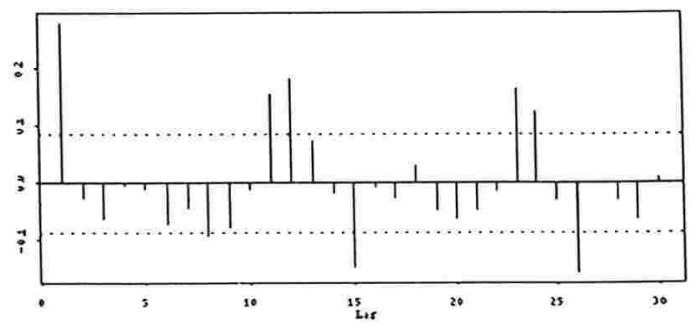

(c)

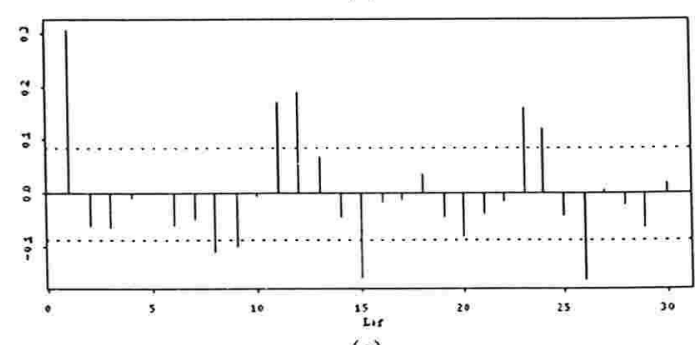

(e)

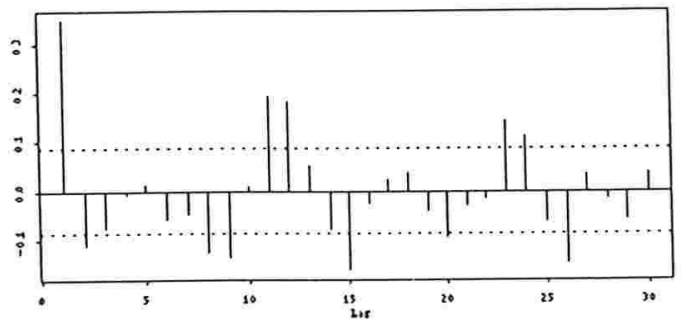

(b)

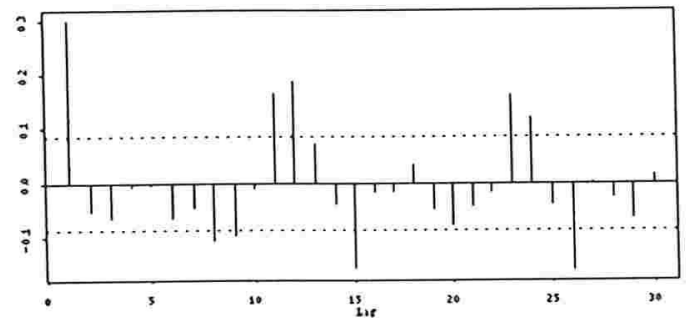

(d)

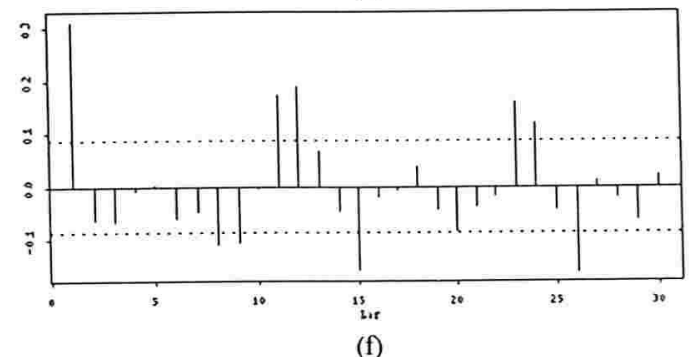

Figura 7.S: Funçào de autocorrelaçào parcial amostral dos resícluos: (a) estimador $\hat{d}_{c}$, (b) estimador $\hat{d}_{c y}$. (c) estimador $\hat{d}_{p}$, (d) estimador $\hat{d}_{p s}$, (e) estimador $\hat{d}_{m v}$, (f) estimador $\hat{d}_{w}$. 
A Tabela i.ł apresenta os resultados das estimativas e desvios padròes dos parâmetros desses modelos. Com base nessa tabela. temos todas as estimativas dos parâmetros significativamente diferentes de zero ao nível se significância de 0.05.

Tabela 7.4 - Resultados do ajuste do modelo $A R F I M A(1, d, 0) \times(0,1,1)_{12}$ à Série de Vazão

\begin{tabular}{|c|c|c|c|c|c|c|c|}
\hline \multirow{2}{*}{$\begin{array}{l}\text { Estimadores } \\
\text { de } d\end{array}$} & \multicolumn{2}{|c|}{ Estimativa } & \multicolumn{2}{|c|}{ Desvio Padrão } & \multicolumn{2}{|c|}{ P-valor } & \multirow[b]{2}{*}{$\hat{\sigma}_{a}^{2}$} \\
\hline & $\hat{\phi}_{1}$ & $\hat{\theta}_{12}$ & $\hat{\phi}_{1}$ & $\hat{\theta}_{12}$ & $\hat{\phi}_{1}$ & $\hat{\theta}_{12}$ & \\
\hline$\hat{d}_{c}=0.3+15$ & 0,2685 & 0,9581 & 0,0420 & 0.0125 & 6.39 & 76.65 & 0.0711 \\
\hline$\hat{d}_{\imath g}=0.3199$ & 0,2736 & 0,9580 & 0,0419 & 0.0125 & 6.52 & 76.64 & 0.071. \\
\hline$\hat{d}_{p}=0.4610$ & 0,2141 & 0,9490 & 0,0425 & $0.013 \overline{1}$ & 2,38 & 69.27 & 0.0679 \\
\hline$\hat{d}_{p s}=0.4172$ & 0,2367 & 0,9534 & 0,0423 & 0,0131 & 5,59 & 72.79 & 0.0690 \\
\hline$\hat{d}_{m v}=0.389 .5$ & 0,2446 & 0,9554 & 0.0422 & 0,0129 & 5,79 & $i+.06$ & $0,0 \bar{\imath}+0$ \\
\hline$\hat{d}_{w}=0,3911$ & 0,2441 & 0,9553 & 0,0422 & 0,0129 & 5,78 & 70.05 & 0,0694 \\
\hline
\end{tabular}

A seguir, são apresentadas na Figura 7.9 a função de autocorrelação amostral dos resíduos do modelo $S . A R F I . M A(1, d, 0) \times(0,1,1)$ 12 para cada um dos seis estimadores do parâmetro d. Da mesma forma, temos as funções de autocorrelação parcial amostral dos resíduos na Figura 7.10 .

Os gráficos da Figura 7.9 mostram que a funçào de autocorrelaçào amostral dos resíduos de ordem 6 é considerada significativamente diferente de zero para todos os métodos de estimação do parâmetro $d$. A função de autocorrelação parcial, Figura 7.10 , apresenta valores altos nos "lags" 6, 13, 23 e 26 para todos os estimadores de $d$. Esse comportamento deve ser melhorado com a inclusão de um parâmetro auto-regressivo de ordem seis nesse modelo. Com isso, ajustamos o modelo $\operatorname{SARFIMA}(6, d, 0) \times(0,1,1)_{12}$, onde $o_{2}=o_{3}=o_{4}=\phi_{5}=0$. Os resultados das estimativas desse modelo sào apresentados na Tabela 7.5 . onde podemos perceber que os parâmetros do modelo $S A R F I M A(6, d, 0) \times(0,1,1)_{12}$ sào significativamente diferentes de zero, ao nível de significância de 0.05 , para todos os métodos de estimação do parâmetro $d$. As funções de autocorrelação e autocorrelação parcial amostrais dos resíduos desse modelo sào dadas nas Figuras 7.11 e 7.12 , respectivamente, para todos os estimadores de $d$. 


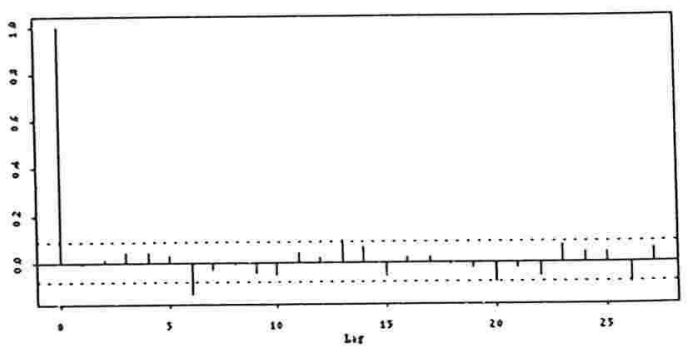

(a)

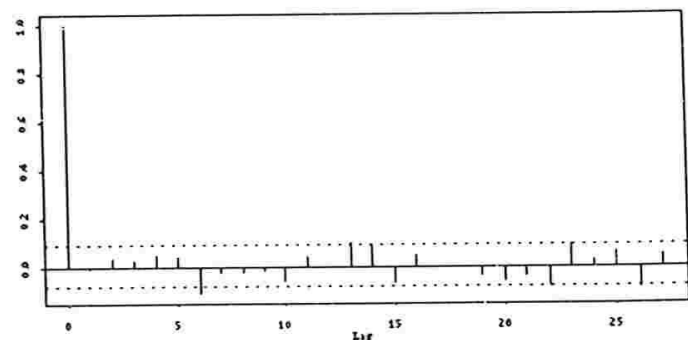

(c)

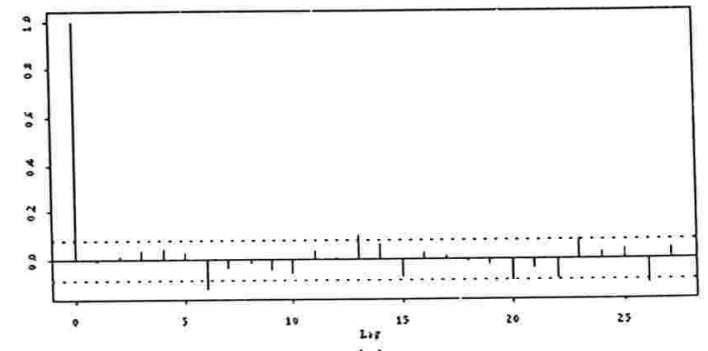

(e)

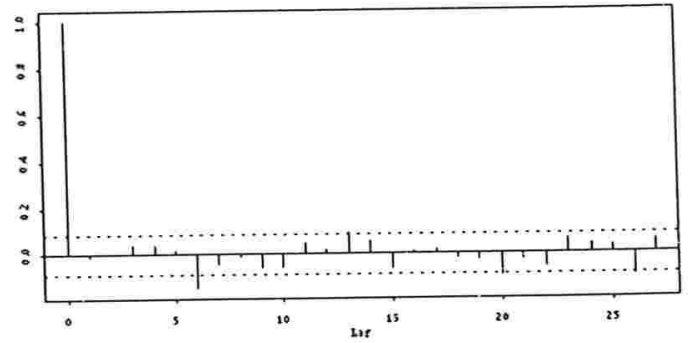

(b)

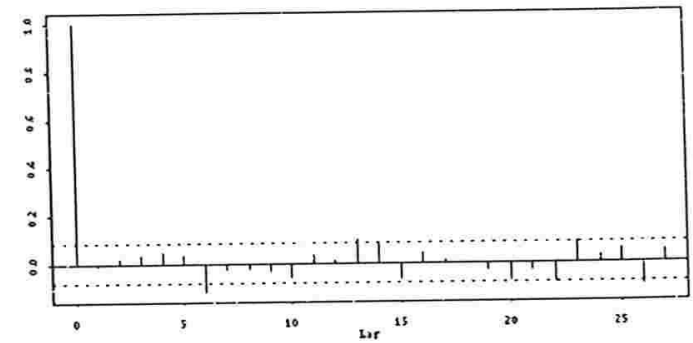

(d)

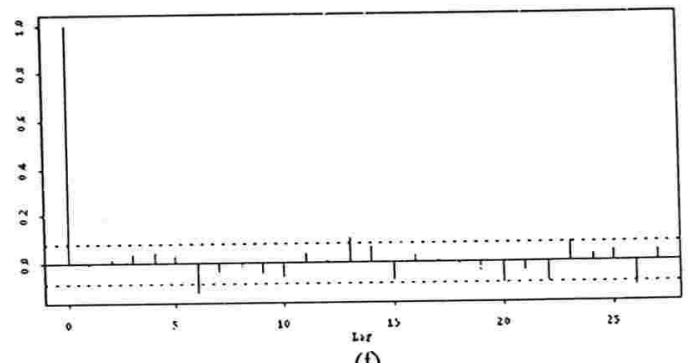

(f)

Figura 7.9: Funçào de autocorrelação amostral dos resíduos: (a) estimador $\hat{d}_{c}$. (b) estimador $\hat{d}_{c g},(\mathrm{c})$ estimador $\hat{d}_{p},(\mathrm{~d})$ estimador $\hat{d}_{p^{\prime}}$. (e) estimador $\hat{d}_{m v},(\mathrm{f})$ estimador $\hat{d}_{u}$. 


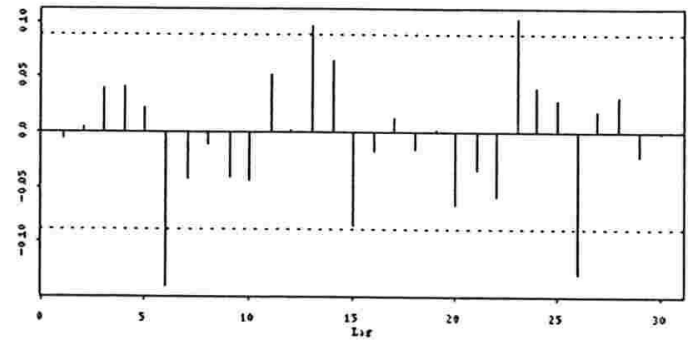

(a)

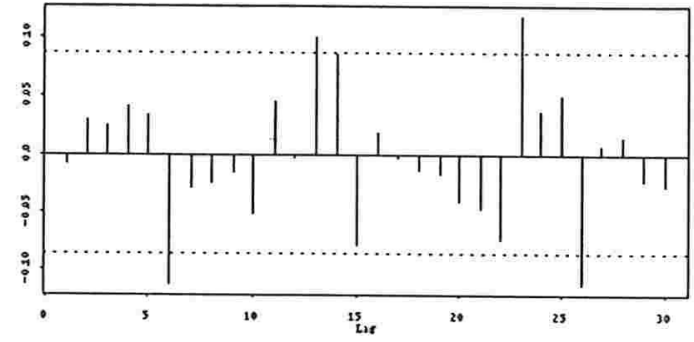

(c)

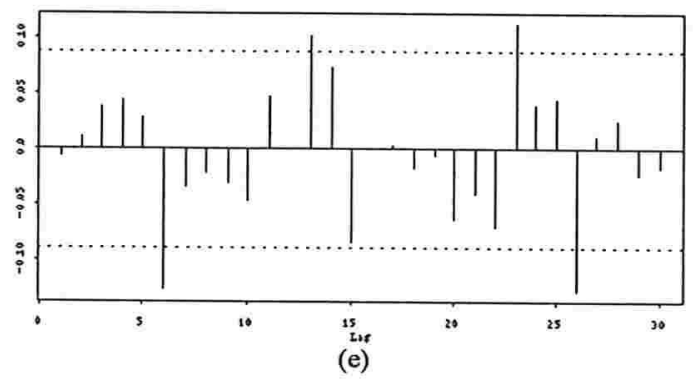

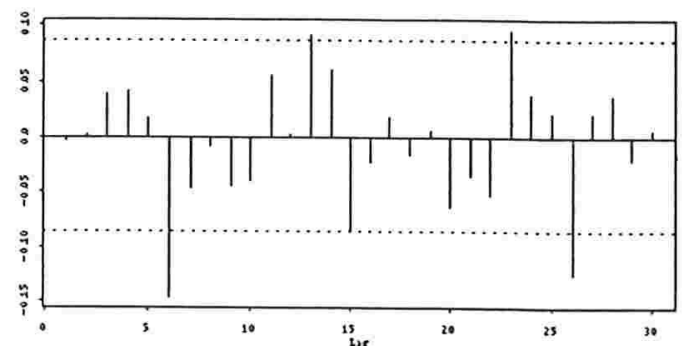

(b)

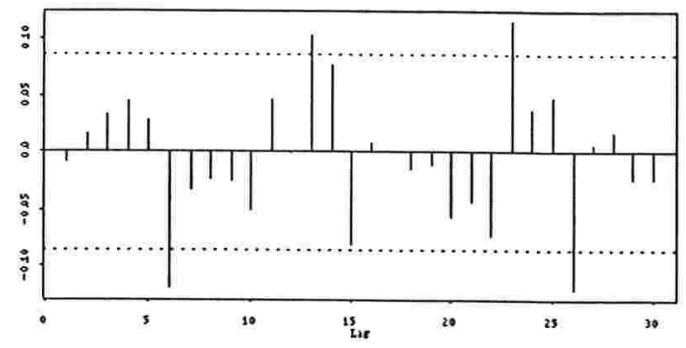

(d)

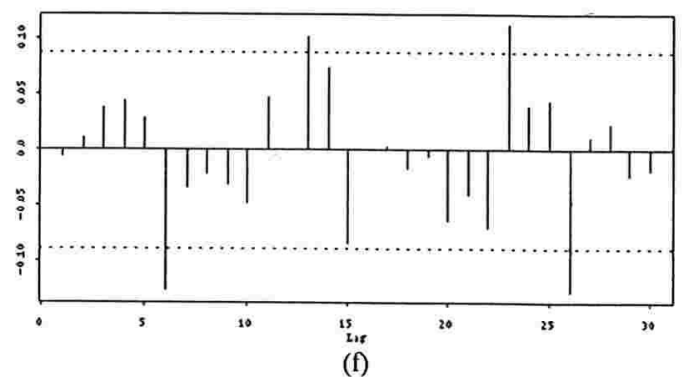

Figura 7.10 : Funçào de autocorrelação parcial amostral dos resíduos: (a) estimador $\hat{d}_{c}$, (b) estimador $\hat{d}_{c g},(\mathrm{c})$ estimador $\hat{d}_{p}$, (d) estimador $\hat{d}_{p s}$, (e) estimador $\hat{d}_{m v}$, (f) estimador $\hat{d}_{w}$. 
Tabela 7.5 - Resultados do ajuste do modelo SARFIM $\mathcal{A}(6, d .0) \times(0,1,1)_{12}$ à Série de Vazão

\begin{tabular}{|l|c|c|c|c|c|c|c|c|c|c|}
\hline \multirow{2}{*}{$\begin{array}{l}\text { Estimadores } \\
\text { de } d\end{array}$} & \multicolumn{3}{|c|}{ Estimativa } & \multicolumn{3}{c|}{ Desvio Padrão } & \multicolumn{3}{c|}{ T-valor } & \\
\cline { 2 - 10 } & $\hat{\phi}_{1}$ & $\hat{\phi}_{6}$ & $\hat{\theta}_{12}$ & $\hat{\phi}_{1}$ & $\hat{\phi}_{6}$ & $\hat{\theta}_{12}$ & $\hat{\phi}_{1}$ & $\hat{o}_{6}$ & $\hat{\theta}_{12}$ & $\hat{\sigma}_{a}^{2}$ \\
\hline$\hat{d}_{c}=0,3162$ & 0,2465 & $-0,1419$ & 0,9043 & 0,0415 & 0,0412 & 0.0132 & 6,70 & -3.46 & 74,72 & 0,0729 \\
$\hat{d}_{c g}=0,3198$ & 0,2771 & $-0,1613$ & 0,9156 & 0,0416 & 0,0413 & 0,0132 & 6,63 & $-3,43$ & 74,74 & 0,0709 \\
$\hat{d}_{p}=0,461$ & 0,1999 & $-0,1169$ & 0,9051 & 0,0425 & 0,0423 & 0,0131 & 5,04 & -2.51 & 74,33 & 0,0682 \\
$\hat{d}_{p s}=0,4172$ & 0,2269 & $-0,0993$ & 0,8846 & 0,0423 & 0,0421 & 0.0131 & 5,47 & -2.68 & 74,82 & 0,0708 \\
$\hat{d}_{m v}=0,4001$ & 0,2672 & $-0,1055$ & 0,8961 & 0,0422 & 0,0420 & 0.0131 & 5,65 & -2.79 & 75,03 & 0,0707 \\
$\hat{d}_{w}=0,4419$ & 0,2481 & $-0,0812$ & 0,9119 & 0,0424 & 0,0422 & 0.0131 & 5,23 & -2.60 & 74,54 & 0,0693 \\
\hline
\end{tabular}

Os gráficos das funçòes de autocorrelação e de autocorrelação parcial amostrais dos resíduos apresentam formas parecidas para todos os estimadores de $d$ e essas duas funções apresentam valores significativamente diferentes de zero no "lag" 26, mas por este "lag" ser de ordem elevada nào consideraremos a inclusão de mais um parâmetro no modelo. 


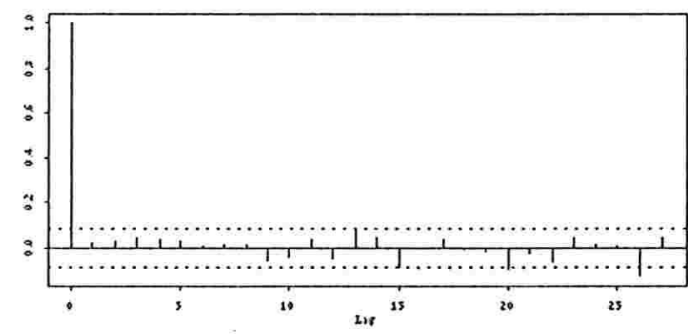

(a)

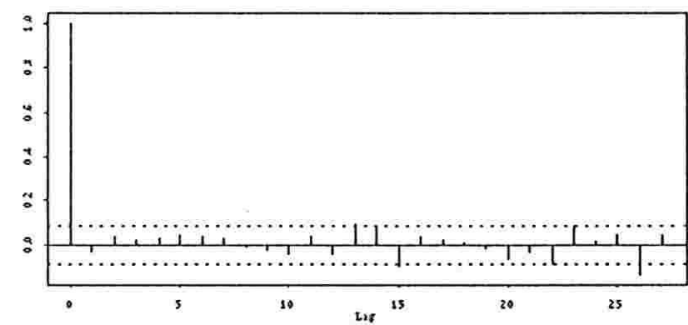

(c)

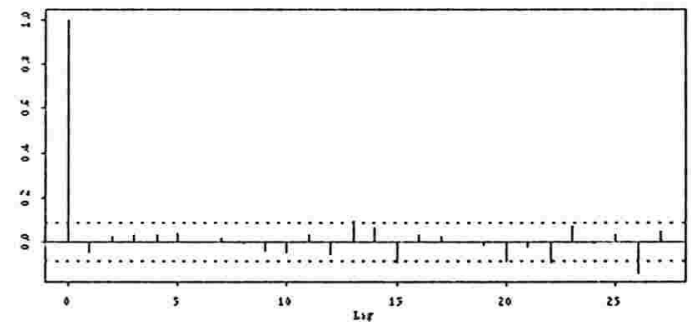

(c)

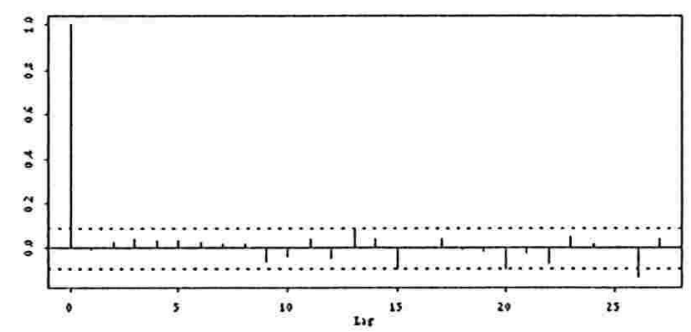

(b)

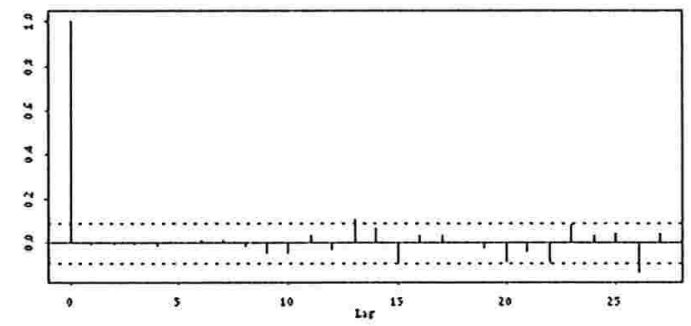

(d)

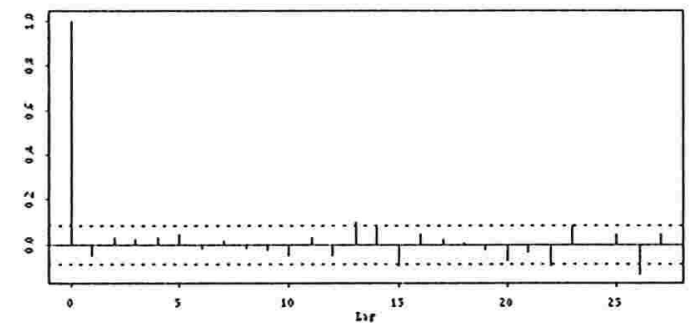

(I)

Figura 7.11: Função de autocorrelação amostral dos resíduos: (a) estimador $\hat{l}_{c}$, (b) estimador $\hat{d}_{c g}$, (c) estimador $\hat{d}_{p},(\mathrm{~d})$ estimador $\hat{d}_{p s},(\mathrm{e})$ estimador $\hat{d}_{m v},(\mathrm{f})$ estimador $\hat{d}_{w}$. 


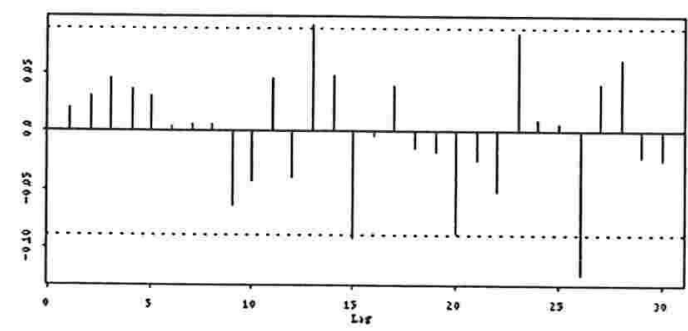

(a)

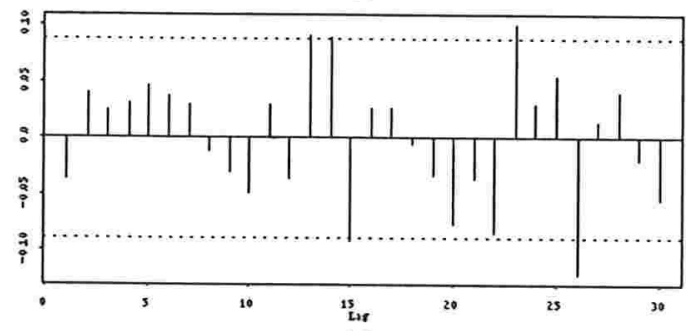

(c)

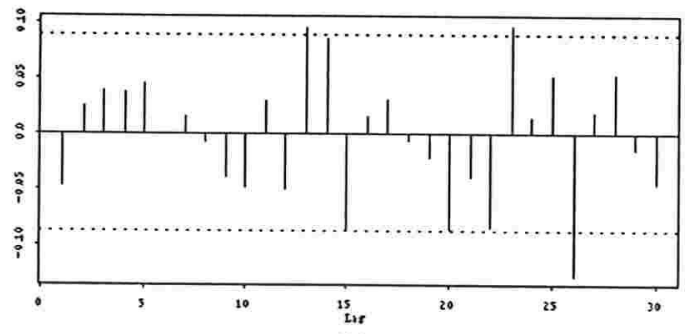

(e)

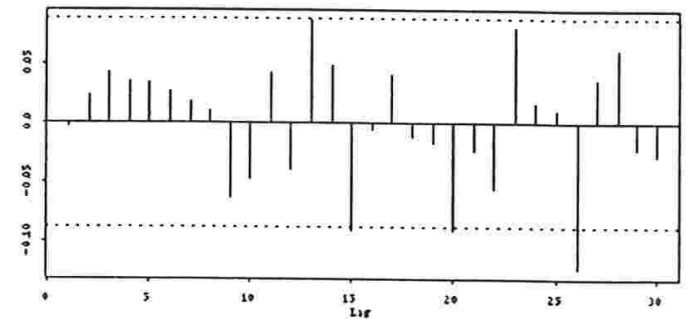

(b)

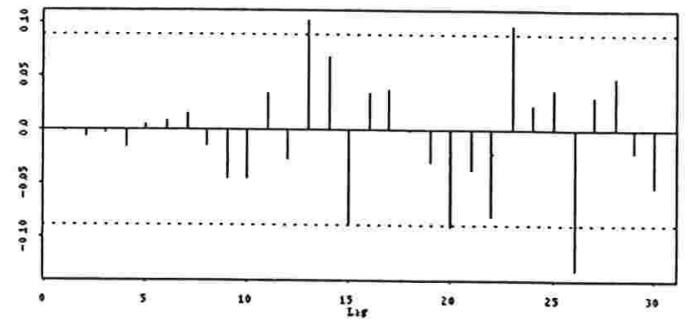

(d)

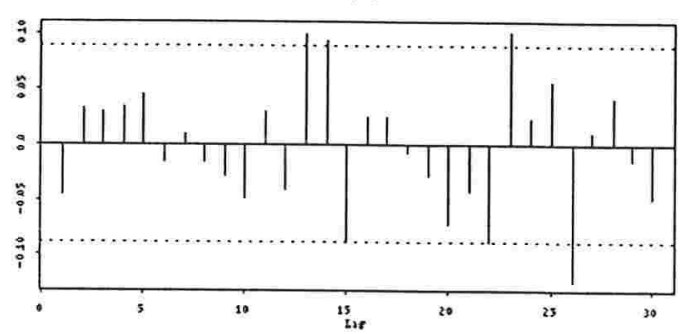

(f)

Figura 7.12: Função de autocorrelação parcial amostral dos resíduos: (a) estimador $\hat{d}_{c}$, (b) estimador $\hat{d}_{c g},(\mathrm{c})$ estimador $\hat{d}_{p},(\mathrm{~d})$ estimador $\hat{d}_{p s},(\mathrm{e})$ estimador $\hat{d}_{m v}$, (f) estimador $\hat{d}_{w}$. 


\subsubsection{Comparação dos modelos}

Para comparar os modelos ajustados à série de vazào do Rio Sào Marcos vamos utilizar uma medida de ajustamento e o potencial de previsão desse modelos.

\section{(i) Ajustamento}

Para mensurar o ajustamento dos modelos usaremos a medida $A I C=-2 \log (L(\Theta))+2 n$, onde $L(\Theta)$ é a verossimilhança do modelo e $n$ é do número de parâmetros estimados. Essa é uma medida comparativa do ajustamento dos modelos, quanto menor for o valor do AIC melhor será o ajuste do modelo. A Tabela 7.6 apresenta essa medida para todos os modelos ajustados à série de vazào.

Tabela 7.6 - Comparação dos modelos através do AIC

\begin{tabular}{|l|r|r|}
\hline Modelos a justados & AIC & $\hat{\sigma}_{a}^{2}$ \\
\hline$A R(25)$ & $1.400,00$ & 0,1033 \\
$S A R I M A(1.0 .0) \times(0,1,1)_{12}$ & 326,60 & 0,1032 \\
$S A R I M A(6.0 .0) \times(1,1,1)_{12}$ & 322,81 & 0,1037 \\
$S A R F I M A(1 . d, 0) \times(0,1.1)_{12}$ & & \\
$\hat{d}_{c}$ & 136.13 & 0,0711 \\
$\hat{d}_{c g}$ & 139,09 & 0,0714 \\
$\hat{d}_{p}$ & $109,8.5$ & 0,0679 \\
$\hat{d}_{p s}$ & 119,01 & 0,0690 \\
$\hat{d}_{m v}$ & 123,22 & 0,0740 \\
$\hat{d}_{w}$ & 118,79 & 0,0694 \\
$S A R F I M A(6 . d, 0) \times(0.1 .1)_{12}$ & & \\
$\hat{d}_{c}$ & 136,88 & 0,0729 \\
$\hat{d}_{c g}$ & 133,97 & 0,0709 \\
$\hat{d}_{p}$ & 108,48 & $0,068.2$ \\
$\hat{d}_{p s}$ & 120,99 & 0,0708 \\
$\hat{d}_{m v}$ & 121,68 & 0,0707 \\
$\hat{d}_{w}$ & 113,29 & 0,0693 \\
\hline
\end{tabular}


Através da Tabela 7.6 podemos notar que o modelo $f R(25)$ foi o que obteve o maior AIC, o que se justifica pela nào significância das estimativas de seus parâmetros. Os modelo $S A R I M A(1,0.0) \times\left((0,1,1)_{12}\right.$ e $S . A R I M A(6.0,0) \times\left((0,1,1)_{12}\right.$ obtiveram valores de AIC muito próximos, indicando que a inclusão do parâmetro $\omega_{6}$ no modelo não alterou muito o ajuste. Além disso, esses valores são cerca de três vezes maiores que os dos modelo de longa memória. Com isso, de acordo com o $\mathrm{AIC}$, os modelos que melhor ajustaram a série foram os $\operatorname{SARFIMA}(1, d, 0) \times(0,1,1)_{12}$ e $S A R F I M A(6, d, 0) \times(0,1,1)_{12}$. Entretanto, para esses modelos, a inclusão do parâmetro $\phi_{6}$ nem sempre implicou numa diminuição do AIC. A variância estimada do modelo sofreu uma redução de cerca de trinta porcento da variância quando utilizamos os modelo de longa memória. Com relação aos estimadores do parâmetro $d$, os modelos que utilizaram os estimadores $\hat{d}_{p}$ e $\hat{d}_{w}$ foram os que obtiveram melhores resultados para o AIC e para a variância estimada e os modelos que utilizaram os estimadores $\hat{d}_{c}$ e $\hat{d}_{c g}$ foram os que obtiveram os piores resultados. Entretanto, essas diferenças são pequenas principalmente com relação à variância estimada.

\section{(ii) Previsão}

Para verificar o poder de previsão dos modelos ajustados utilizamos a série de vazão sem as doze últimas observações para fazer a previsão de doze meses. Com isso, iremos comparar os valores previstos com os valores reais e utilizaremos o erro quadrático médio (EQM) dessas previsões para comparar os modelos. A seguir, apresentamos a Tabela 7.7 com os valores previstos juntamente com os valores reais e a Tabela $7.8 \mathrm{com}$ os erros de previsão dados pela diferença entre o valor real e o valor previsto pelos modelos. 
Tabela 7.7 - Previsão dos modelos ajustados

\begin{tabular}{|c|c|c|c|c|c|c|c|c|c|c|c|c|}
\hline \multirow[t]{2}{*}{ Modelos ajustados } & \multicolumn{12}{|c|}{ Valores Previstos } \\
\hline & $Y_{t+1}$ & $Y_{t+2}$ & $Y_{t+3}$ & $\dot{Y}_{t+4}$ & $\dot{y}_{t+5}$ & $\dot{r}_{t+6}$ & $\dot{Y}_{t+i}$ & $i_{t+8}$ & $Y_{i+9}$ & $\hat{Y}_{t+10}$ & $\hat{Y}_{t+11}$ & $\hat{Y}_{t+12}$ \\
\hline$A R(25)$ & 54,3 & 38,3 & 33.5 & 25,7 & 15.9 & 9,9 & 7.9 & 5.6 & 4.9 & 5.8 & 8,2 & 8,6 \\
\hline$S A R I M A$ & & & & & & & & & & & & \\
\hline$(1,0,0) \times(0,1,1)_{12}$ & 175,1 & 100,7 & 58,9 & 34,0 & 26.0 & 18,2 & 17,2 & 12,8 & 13.4 & 19.3 & 2,8 & 5,4 \\
\hline$(6,0,0) \times(1,1,1)_{12}$ & 48,0 & 89,0 & 66.8 & 32,5 & 36,0 & 24,9 & 21,8 & 19,7 & 32.0 & 35,6 & 63,1 & 68,1 \\
\hline $\begin{array}{l}\text { S.ARFIMA } \\
\quad(1, d, 0) \times(0,1,1)_{12}\end{array}$ & & & & & & & & & & & & \\
\hline$\dot{d}_{c}$ & 371,5 & 333,0 & 170,8 & 108,0 & 88,8 & 66,8 & 62,6 & 42,6 & $50, \bar{t}$ & 67.1 & 8,7 & 22,4 \\
\hline$\dot{d}_{c g}$ & 352,9 & 312,4 & 159,9 & 101,0 & 82,9 & 62,3 & 58,4 & 39.8 & 47.3 & 62,6 & 8,1 & 20,9 \\
\hline$\dot{d}_{p}$ & 394,8 & 363,1 & 212.0 & 141,6 & 118.3 & 89,1 & 85,0 & 60,4 & 69.1 & 95.0 & 12,5 & 29,0 \\
\hline$\hat{d}_{p s}$ & 393,2 & 361,2 & 199.1 & 129,9 & 107,9 & 82,3 & 76.9 & 53.6 & 62.4 & 84,4 & 11,0 & 26,8 \\
\hline$\dot{d}_{m v}$ & 386,9 & 353,3 & 189.3 & 122,0 & 101.0 & 76.0 & 71,6 & 49.5 & 58.1 & $7 \pi, 8$ & 10.1 & 25,3 \\
\hline$\hat{d}_{w}$ & 387,4 & 353,9 & 189.9 & 122,5 & 101.4 & 76,3 & 72,0 & 49,7 & 58.4 & 78.2 & 10,2 & 25,4 \\
\hline$(6, d, 0) \times(0,1,1)_{12}$ & & & & & & & & & & & & \\
\hline $\begin{array}{l}d_{c} \\
\dot{d}_{c}\end{array}$ & 227.4 & 159,2 & 131.6 & 84.8 & 87.0 & 60.7 & 58.0 & 46,3 & 43.6 & 62.0 & 10,0 & 15,0 \\
\hline$d_{c g}$ & 245,2 & 177.5 & 131,7 & 82,7 & 88.5 & 61,6 & 59.0 & 46.1 & 55.4 & 65.7 & 9,7 & $15, \bar{i}$ \\
\hline$d_{p}$ & 280,7 & 219,4 & 192.0 & 130,4 & $132, \pi$ & 93,6 & 88,5 & 69,9 & 65.9 & 93.5 & 15,2 & 23,0 \\
\hline$d_{p s}$ & 235,3 & 170,8 & 173.5 & 119,5 & 114,3 & 81,8 & 77,1 & 62.8 & 55.8 & 75,7 & 14,5 & 19,7 \\
\hline$d_{m v}$ & 248,4 & 182,7 & 168,7 & 114,4 & 111,0 & 79,3 & 75,0 & 60,2 & 55.0 & 76,6 & 13,3 & 19,1 \\
\hline$\hat{d}_{w}$ & 292,3 & 231,0 & 189,8 & 129,9 & 123,6 & 88,5 & 84,1 & 65,8 & 63.4 & 90.5 & 14,1 & 22,4 \\
\hline Valores Reais & 185,0 & 210,0 & 114.0 & 142,0 & 101,0 & 73,0 & 61,0 & 43.0 & 30.0 & 41,0 & 97,0 & 114,0 \\
\hline
\end{tabular}

Tabela 7.8 - Erros de previsão dos modelos ajustados

\begin{tabular}{|c|c|c|c|c|c|c|c|c|c|c|c|c|}
\hline \multirow[t]{2}{*}{ Modelos ajustados } & \multicolumn{12}{|c|}{ Erros de previsào (Valor Real - Valor previsto) } \\
\hline & $\hat{Y_{t+1}}$ & $\hat{Y}_{t+2}$ & $\hat{Y}_{t+3}$ & $\hat{Y}_{t+4}$ & $\hat{Y}_{t+5}$ & $\dot{Y_{t+6}}$ & $r_{t+7}$ & $\hat{Y}_{t+8}$ & $\dot{Y}_{t+3}$ & $Y_{t+10}$ & $\dot{Y}_{t+11}$ & $\dot{Y}_{t+12}$ \\
\hline $\begin{array}{l}A R(25) \\
\text { SARIMAA }\end{array}$ & 130,6 & 171,7 & 80.5 & 116,3 & 85,1 & 63,0 & 53,1 & 37,4 & 25.1 & 35.2 & 88,8 & 105,4 \\
\hline$(1,0,0) \times(0,1,1)_{12}$ & 9,8 & 109,2 & 55.1 & 108,0 & 75.0 & 54,7 & 43.8 & 30,2 & 16.6 & 21,7 & 94.1 & 108,6 \\
\hline$(6,0,0) \times(1,1,1)_{12}$ & 137,0 & 121,0 & 47,2 & 109,5 & 65.0 & 48,1 & 39,2 & 23,3 & $-2,0$ & 5.4 & 33,9 & 45,9 \\
\hline $\begin{array}{l}\text { SARFIMA } \\
\quad(1, d, 0) \times(0,1,1)_{12}\end{array}$ & & & & & & & & & & & & \\
\hline$\hat{d}_{c}$ & $-186,5$ & -123.0 & -56.8 & 34,0 & 12.2 & 6.2 & -1.6 & 0.34 & $-20 . \overline{7}$ & -26.1 & 88,3 & 91,6 \\
\hline$\hat{d}_{c g}$ & $-167,9$ & $-102,4$ & $-45,9$ & 41,0 & 18,1 & 10,7 & 2,6 & 3,2 & -17.3 & $-21,6$ & 88,9 & 93,1 \\
\hline$\hat{d}_{p}$ & $-209,8$ & $-153,1$ & $-98,0$ & 0,4 & -17.3 & $-16,1$ & $-24,0$ & $-17,4$ & -39.1 & $-54,0$ & 84,4 & 85,0 \\
\hline$\hat{d}_{p s}$ & $-208,2$ & $-151,2$ & -85.1 & 12,1 & -6.9 & -8.2 & $-15,9$ & $-10,6$ & -32.4 & -43.4 & 86,0 & $8 \pi, 1$ \\
\hline$\dot{d}_{m v}$ & $-202,0$ & $-143,3$ & -75.3 & 20,0 & 0.03 & $-3,0$ & $-10,6$ & $-6,4$ & -28.1 & -36.8 & 86,9 & $88, \pi$ \\
\hline $\begin{array}{c}\dot{d}_{w} \\
(6, d, 0) \times(0,1,1)_{12}\end{array}$ & $-202,4$ & $-143,9$ & -75.9 & 19,5 & $-0,4$ & $-3,3$ & $-11,0$ & $-6, i$ & $-28,4$ & $-37,2$ & 86,8 & 88,6 \\
\hline$i_{c}$ & $-42,4$ & 50,7 & $-1 \bar{i} .6$ & 57,2 & 14.0 & 12,3 & 2,9 & $-3,3$ & -13.6 & $-21,0$ & 87,0 & 99,0 \\
\hline$\hat{d}_{c g}$ & $-60,3$ & 32,5 & $-17,7$ & 59,3 & 12,5 & 11,4 & 2,0 & $-3,1$ & $-15,4$ & $-24,7$ & 87,3 & 98,3 \\
\hline$\hat{d}_{p}$ & $-96,0$ & $-9,4$ & -78.0 & 11,6 & -31.2 & $-20,6$ & $-27,5$ & $-2 \tau, 0$ & -35.9 & -52.5 & 81,8 & 91,0 \\
\hline$\hat{d}_{p s}$ & $-50,3$ & 39,2 & -59.5 & 22,5 & $-13,3$ & $-8,8$ & $-16,1$ & $-19,8$ & -25.8 & -34.7 & 82,5 & 94,3 \\
\hline$\dot{d}_{m v}$ & $-63,3$ & 27,3 & -54.7 & 27,6 & -10.0 & $-6,3$ & $-14,0$ & $-17,2$ & -25.0 & $-35,6$ & 83,7 & 94,9 \\
\hline$\hat{d}_{w}$ & $-107,3$ & $-21,0$ & -75.8 & 12,1 & $-22,6$ & $-15,5$ & $-23,1$ & $-22,8$ & -33.4 & -49.4 & 82,9 & 91,6 \\
\hline
\end{tabular}


Com base nas Tabelas 7.7 e 7.8 . podemos dizer que os modelos $A R$ e $S . A R I M A$ ajustados oferecem previsões, em geral. abaixo dos valores reais. Ou seja. esses modelos subestimam as previsões. Também podemos perceber que os valores previstos pelos modelos $S^{\prime} A R F I M A(6, d, 0) \times(0,1,1)_{12}$ estào bem mais próximos dos valores reais que os previstos pelos modelos $S A R F I M A(1, d, 0) \times(0,1,1)_{12}$. Para comparar o poder de previsão desses modelos utilizamos a média dos erros ao quadrado (erro quadrático médio das previsões), dados na Tabela 7.9 .

Tabela 7.9 - Erro quadrático médio das previsões

\begin{tabular}{|l|r|}
\hline Modelos a justados & EQM \\
\hline$A R(25)$ & 8573.323 \\
$S A R I M A$ & \\
$(1.0,0) \times(0,1,1)_{12}$ & 4965.775 \\
$(6,0,0) \times(1,1,1)_{12}$ & 4961.309 \\
$S A R F I M A$ & \\
$(1, d, 0) \times(0,1,1)_{12}$ & \\
$\hat{d}_{c}$ & 5981.006 \\
$\hat{d}_{c g}$ & 5022,499 \\
$\hat{d}_{p}$ & 8108.092 \\
$\hat{d}_{p s}$ & 7666.891 \\
$\hat{d}_{m v}$ & 7095.606 \\
$\hat{d}_{w}$ & 7132.336 \\
$(6, d, 0) \times(0,1,1)_{12}$ & \\
$\hat{d}_{c}$ & 2193.812 \\
$\hat{d}_{c g}$ & 2245.251 \\
$\hat{d}_{p}$ & 3114,370 \\
$\hat{d}_{p s}$ & 2216.286 \\
$\hat{d}_{m v}$ & 2253.999 \\
$\hat{d}_{w}$ & 3204.861 \\
\hline
\end{tabular}

Comparando os modelos ajustados através da Tabela 7.9 , podemos dizer que segundo o erro quadrático médio das previsões o pior modelo é o $\operatorname{AR}(25)$. seguido dos modelos $S A R F I M A\left((1,0,0) \times(0,1,1)_{12}\right.$. Os modelos $S . A R I M A(1.0,0) \times(0,1.1)_{12}$ e $S . A R I M A(6,0,0) \times$ $(1,1,1)_{12}$ são melhores que estes últimos, porém piores que os modelos S.ARFI.MA(6,d,0)× 


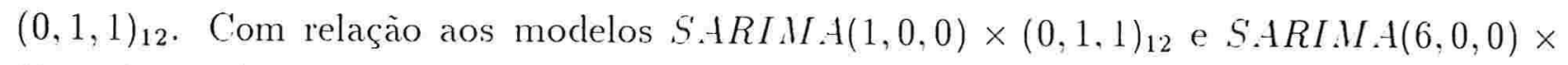
$(1,1,1)_{12}$ podemos perceber que a inclusào do parâmetro $\phi_{6}$ não melhora o poder de previsão do modelo SARIMA, ao contrario dos modelos de longa memória, onde a inclusão deste parâmetro diminui cerca de 50 porcento o erro quadrático médio das previsões. Com isso os melhores modelos, segundo a previsão, são os modelos $S A R F I M A(6, d, 0) \times(0,1,1)_{12} \mathrm{e}$ dentre estes modelos os que apresentaram menor erro quadrático médio foram os que utilizaram $\hat{d}_{c}, \hat{d}_{c g}, \hat{d}_{p s}$ e $\hat{d}_{m v}$ como estimadores de $d$.

\section{Conclusão:}

- De uma forma geral, os modelos S.tRFI.MA(6,d.0) $\times(0.1 .1)_{12}$ são os que fornecem melhor ajustamento e previsão. Entretanto, o método utilizado na estimação do parâmetro $d$ não influenciou de forma marcante na qualidade do ajustamento e da previsão.

- $\mathrm{O}$ ajustamento do modelo $\mathrm{AR}(25)$ fornece um modelo super parametrizado que foi péssimo tanto para o ajustamento quanto para obter previsões.

- A utilização de modelos de Box \& Jenkins clássicos, $S A R I M A(p, d, q) \times(P, D, Q)_{12}$, não conseguiu captar o comportamento de longa memória da série. 


\section{Capítulo 8}

\section{Conclusão}

A elaboraçào deste trabalho teve como objetivo comparar os estimadores do parâmetro $d$ do modelo ARFI.MA $(p, d, q)$ diante de diferentes situações que pudessem testar esses estimadores de maneira mais eficiente possível. Os resultados das simulações de amostras de modelos $A R F I M A(0, d, 0)$ para diferentes valores de $d$ e para três tamanhos de amostra mostram quais estimadores pioram suas estimativas a medida que o verdadeiro valor do parâmetro $d$ se aproxima do limite de não estacionariedade, 0,5 . Além disso. os diversos tamanhos de amostra utilizados nos ajudam perceber se esses estimadores melhoram suas estimativas de forma diferenciada quando aumentamos a amostra. A utilização dos diversos valores de $d$ nos permitiu perceber que quando o verdadeiro valor do parâmetro $d$ se aproxima do limite de nào estacionariedade o viés clos estimadores $\hat{d}_{c} \cdot \hat{d}_{c g}, \hat{d}_{m u}$ e $\hat{d}_{w}$ se tornam maiores. Entretanto. esse comportamento tende a diminuir a medida que aumentamos o tamanho da amostra. com excessão dos estimadores $\hat{d}_{c}$ e $\hat{d}_{c g}$ que mantem esse comportamento independente do tamanho da amostra. Com relação aos três tamanhos de amostra utilizados. percebemos que os estimadores $\hat{d}_{p}$ e $\hat{d}_{p s}$ melhoram suas estimativas em proporçòes menores em relação aos demais estimadores, pois para os demais estimadores dobrar o tamanho da amostra implicou numa diminuição de cerca de $50 \%$ do $E \hat{Q} M(\hat{d})$, enquanto que para $\hat{d}_{p}$ e $\hat{d}_{p s}$ essa diminuição foi em torno de $33 \%$. Quanto à classificação geral dos estimadores no modelo $A R F I M A(0, d, 0), \hat{d}_{m v}, \hat{d}_{c}$ e $\hat{d}_{c g}$ formam o grupo dos melhores estimadores, o estimador $\hat{d}_{w}$ se mostrou um estimador intermediário e $\hat{d}_{p}$ e $\hat{d}_{p s}$ foram classificados como os piores, sendo que $\hat{d}_{p}$ é o pior deles. 
Nas simulações do modelo $A R F I M A(1, d, 0)$. onde $d=0.3$ e $o_{1}=0.7$. o valor de $d$ foi escolhido propositalmente por ser um valor afastado do limite de nào estacionariedade. A inclusào do parâmetro auto-regressivo de ordem um teve como objetivo testar se as estimativas do parâmetro $d$ seriam prejudicadas ou se haveria alguma mudança na classificaçào dos estimadores. Também havia interesse em comparar as estimativas do parâmetro $\phi_{1}$ para os diversos métodos de estimação. Como o algorítmo de estimação dos parâmetros do modelo $A R F I M A(p, d, q)$, quando utilizamos $\hat{l}_{p}$ e $\hat{d}_{p s}$, é diferente do algoritmo utilizado no caso dos demais estimadores, esperávamos que houvesse alguma modificação nos resultados finais das estimativas. Entretanto, poucas modificações foram percebidas, apenas o estimador $\hat{d}_{m v}$, que no modelo $A R F I M A(0, d, 0)$ foi classificado como o melhor estimador. neste caso apresentou resultados piores que os de $\hat{d}_{w}$ e foi classificado em quarto lugar. Com isso, temos que os estimadores $\hat{d}_{c}$ e $\hat{d}_{c g}$ continuaram sendo os melhores estimadores. $\hat{d}_{u}$, foi classificado como o terceiro melhor estimador e $\hat{d}_{p}$ e $\hat{d}_{p s}$ foram os piores estimadores. As estimativas do parâmetro $\phi_{1}$ pouco se alteraram em relação ao método de estimação de $d$ utilizado, mas percebemos que os métodos de estimaçào dos parâmetros que apresentaram os melhores resultados para as estimativas de $d$ ofereceram as piores estimativas para $\phi_{1}$ e vice-versa.

Quanto ao modelo $A R F I M A(0, d, 1)$, onde $d=0,3$ e $\theta_{1}=-0,6$, a única mudança observada também foi em relação ao estimador $\hat{d}_{m v}$, que para amostras pequenas não ofereceu o melhor resultado sendo classificado em terceiro lugar. Entretanto, com o aumento da amostra seus resultados se aproximam dos de $\hat{d}_{c}$ e $\hat{d}_{c g}$ que novamente sào os melhores estimadores do parâmetro $d$. Para tamanhos de amostras grandes os estimadores $\hat{d}_{w}$ e $\hat{d}_{p s}$ apresentaram resultados semelhantes e são classificados como estimadores intermediários. A pior classificação foi a de $\hat{d}_{p}$.

Como resultado geral das simulações, temos que os melhores métodos de estimaçãos são os que utilizam $\hat{d}_{c}$ e $\hat{d}_{c g}$, onde $\hat{d}_{c}$ é o mais simples de todos os estimadores. O estimador $\hat{d}_{m v}$ é muito adequado para o modelo $A R F I M A(0, d .0)$, entretanto, suas estimativas se tornam deficientes a medida que outros parâmetros vão sendo incorporados ao modelo. $O$ estimador $\hat{d}_{w}$ apresentou sempre resultados intermediários, enquanto $\hat{d}_{p}$ sempre foi o pior dos estimadores. O estimador $\hat{d}_{p s}$, apresentou resultados significativamente melhores que os de $\hat{d}_{p}$, contudo, essa melhora não foi suficiente para promovê-lo a categoria dos bons estimadores.

Outra forma de comparar os estimadores foi utilizar uma série real para ajustar uma 
modelo ARFIMA(p.d.q) utilizando todos os métodos de estimaçào de $d$. Além disso, ajustamos os modelos $A R(p)$ e $A R I M A(p, d . q)$ à série para poder comparar o modelo de longa memória a esses modelos mais tradicionais. A comparaçào dos modelos revelou a superioridade do modelo $5 . A R F I . M A(p, d, q)$ que ofereceu o melhor ajuste e as melhores previsões. O modelo auto-regressivo ajustado apresentou os piores resultados e de forma alguma indicou que este modelo deva ser usado para ajustar séries de longa memória. $\mathrm{O}$ modelo $A R I M A(p, d, q)$, apesar de ser bem melhor que o $A R(p)$. não pode ser indicado para o ajuste de séries com comportamento de longa memória. Quanto a comparação dos métodos de estimação dos parâmetros do modelo ARF $I M A(p, d . q)$, temos poucas diferenças de resultados entre os métodos de estimação, mas podemos dizer que com relação ao ajuste os melhores estimadores foram $\hat{d}_{p}$ e $\hat{d}_{w}$, em segundo lugar temos $\hat{d}_{p s}$ e $\hat{d}_{m v}$ e $\hat{d}_{c}$ e $\hat{d}_{c g}$ apresentaram o pior ajuste. Entretanto. com relaçào ao poder de previsào. os melhores métodos foram aqueles que utilizaram os estimadores $\hat{d}_{c}, \hat{d}_{c g}$. $\hat{d}_{p s}$ e $\hat{d}_{m v} ; \hat{d}_{p}$ e $\hat{d}_{w}$ apresentaram previsões semalhantes, porém nào tão boas quanto as dos demais estimadores. 


\section{Bibliografia}

Agiakloglou, C.. Newbold, P. and Wohar (1993). Bias in an estimator of the fractional difference parameter. Journal of Time Series Analysis. 14:235-246.

Beltrão. K. I. and Bloomfield. P. (1987). Determinig the bandwidth of a kernel spectrum estimate. Journal of Time Series Analysis, 8:21-38.

Beltrão, K. I. and Hurvich, C. M. (1993). Assymptotics for the low-frequency ordinates of the periodogram of a long memory time series. Journal of Time Series Analysis, 14:45:5-472.

Beran. J. (1992a). A goodness of fit test for time series with long range dependence. J. $R$. Statist. Soc. Ser.. B54:749-760.

Beran. J. (1992b). Statistical methods for data with long range dependence. Stat. Sci.. 7:404-427.

Beran. J. and Terrin, N. (1994). Estimation of long-memory parameter, based on a multivariate central limit theorem. Journal of Time Series Analysis.

Chen, G., Abraham, B. and Peiris, S. (1994). Lag window estimation of the degree of differencing in fractionally integrated time series models. Journal of Time Steries Analysis.

Cheung. Y.W. (1993). Testes for fractional integration: A Monte Carlo investigation. Journal of Time Series Analysis.

Daubechies, I. (1988). Orthonormal bases of compactly sopported wavelets. Communications on Pure and Applied Mathemetics, 41:909-996. 
Daubechies, I. (1992). Ten lectures on wavelets. Philadelphia: SIAM.

Diebold, F.X. and Rudebusch, G.D. (1989). Long memory and persistence in aggregate output. Journal of Monetary and Economics, 24:18.9-206.

Fox, R. and Taqqu, M.S. (1986). Large sample properties of parameter estimates for strongly dependent stationary gaussian time series. The Annals of Statistics.

Geweke, J. and Porter-Hudak. S. (1983). The estimation and application of long memory time series models. Journal of Time Series Analysis, 4:221-238.

Granger, C.M.G. and Joyeux, R. (1980). An introduction to long memory time series models and fractional differencing. Journal of Time S'ries Analysis. 1:15-29.

Gupta, S.N. and Ji. X. (1992). Modeling persistence in time series clata. Estadistica.

Gupta, S.N. and Kumar, A. (1991). Time domain estimation in fractionally differenced noise processes. Journal of Combinatory Information and System Science.

Hassler, U. (1993). Regression of spectral estimator with fractionally integrated time series. Journal of Time Series Analysis, 14:369-380.

Hosking, J.R.M. (1981). Fractional differencing. Biometrika, 68:16:5-176.

Hosking, J.R.M. (1984). Modeling persistence in hydrological time series using fractional differencing. Water Resources Res., 20:1898-1908.

Hurvich, P.M. (1987). Data-driven choice of a spectrum estimates extending the applicability of cross-validation methods. J. Am. Statist. Assoc., 80:933-940.

Jensen, M.J. (1996). Ordinary least squares estimate of the fractional differencing parameter using wavelets. Department of Economics. Southern Illinois University. Preprint

Mandelbrot, B.B. and Ness, V. (1968). Fractional Brownian motion, fractional noises and applications. SIAM, Rev.

Nason, G.P. and Silvermann, B.W. (1994). The discrete wavelet transform in S. Journal of Computational and Graphical Statistics 
Porter-Hudak. S. (1990). An application to the seasonal fractionally differenced model to the monetary aggregates. J. Amer. Statist. Assoc., 85:338-344.

Reisen, V.A. (1994). Estimation of the fractional differenced parameter in the ARIM.A(p,d,q) model using the smoothed periodogram. Journal of Time Serits Analysis. 85:338-344.

Vinod, H. D. (1991). Estimating fractionally integrated ARMA models persistence in GNP. Preprint.

Yajima, Y. (1985). On estimation long memory time series models. Austral. J. Statist., 27:30:3-320. 


\section{Apêndice A}

\section{Programas para estimação dos parâmetros}

1- Programa para a estimação dos parâmetros do modelo $\operatorname{SARFIMA}(6, d, 0) \times(0,1,1)_{12}$ quando o estimador de $d$ é $\hat{d}_{c}$ Método Iterativo

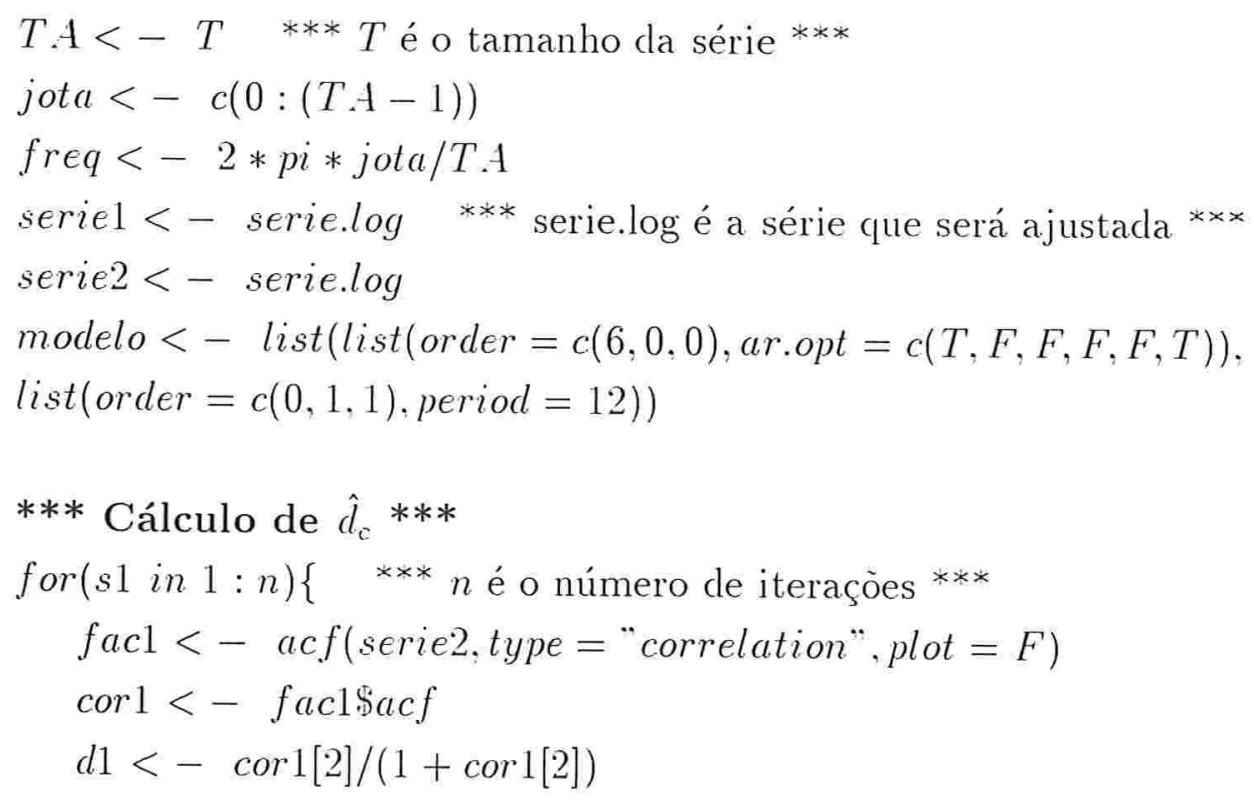




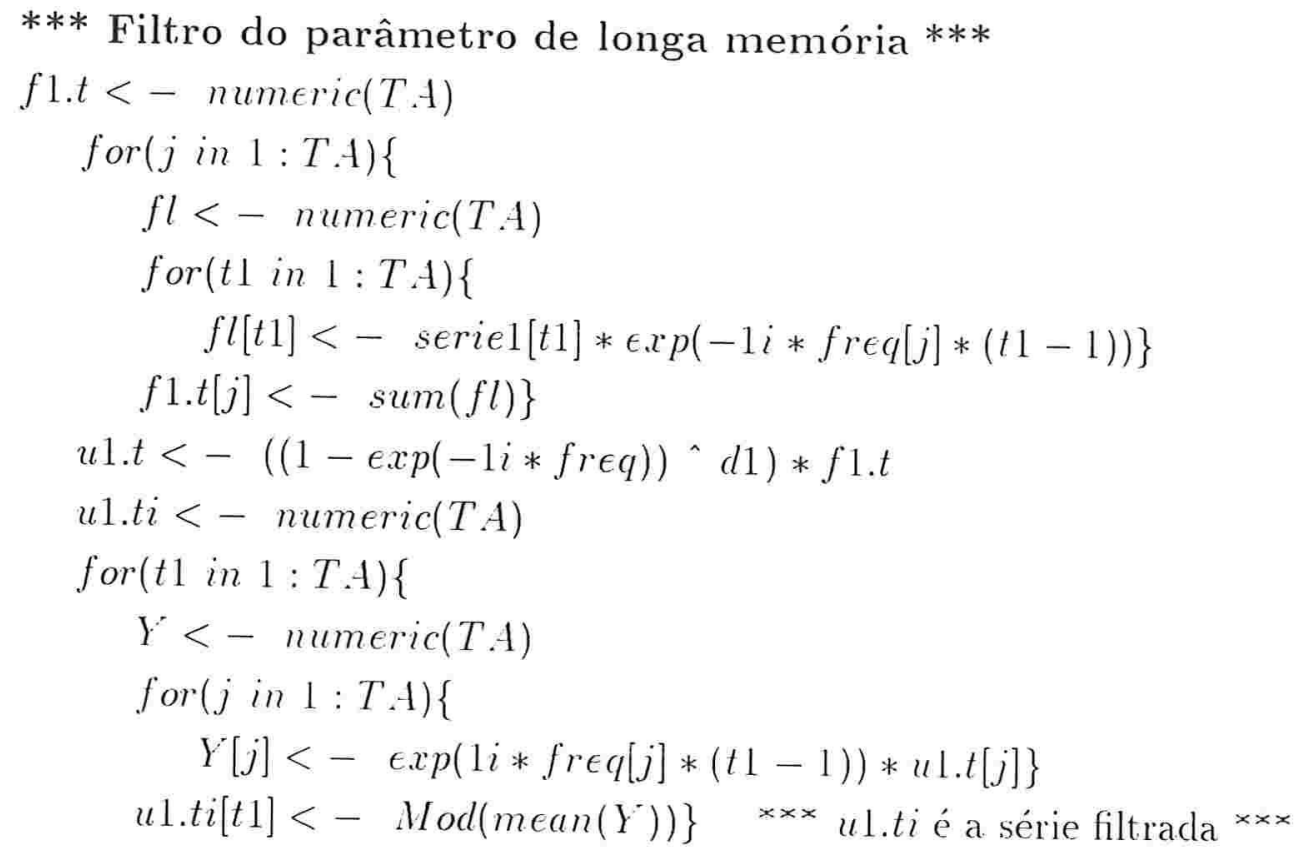

*** Ajuste do modelo $S A R I M A(6,0,0) \times(0,1,1)_{12}$ à série filtrada, u1.ti. *** fit $1<-$ arima.mle $(u 1 . t i$, model $=$ modelo $)$

filtrol $<-$ arima.filt(serie1, model $=$ fit 1 \$model $)$

serie $2<-$ filtrol\$filt $[31:$ T.A] - filtrolspred $[31: T . A]\}$ 
2- Programa para a estimação dos parâmetros do modelo $S A R F I M A(6, d, 0) \times(0.1,1)_{12}$ quando o estimador de $d$ é $\hat{d}_{c g}$ Método Iterativo

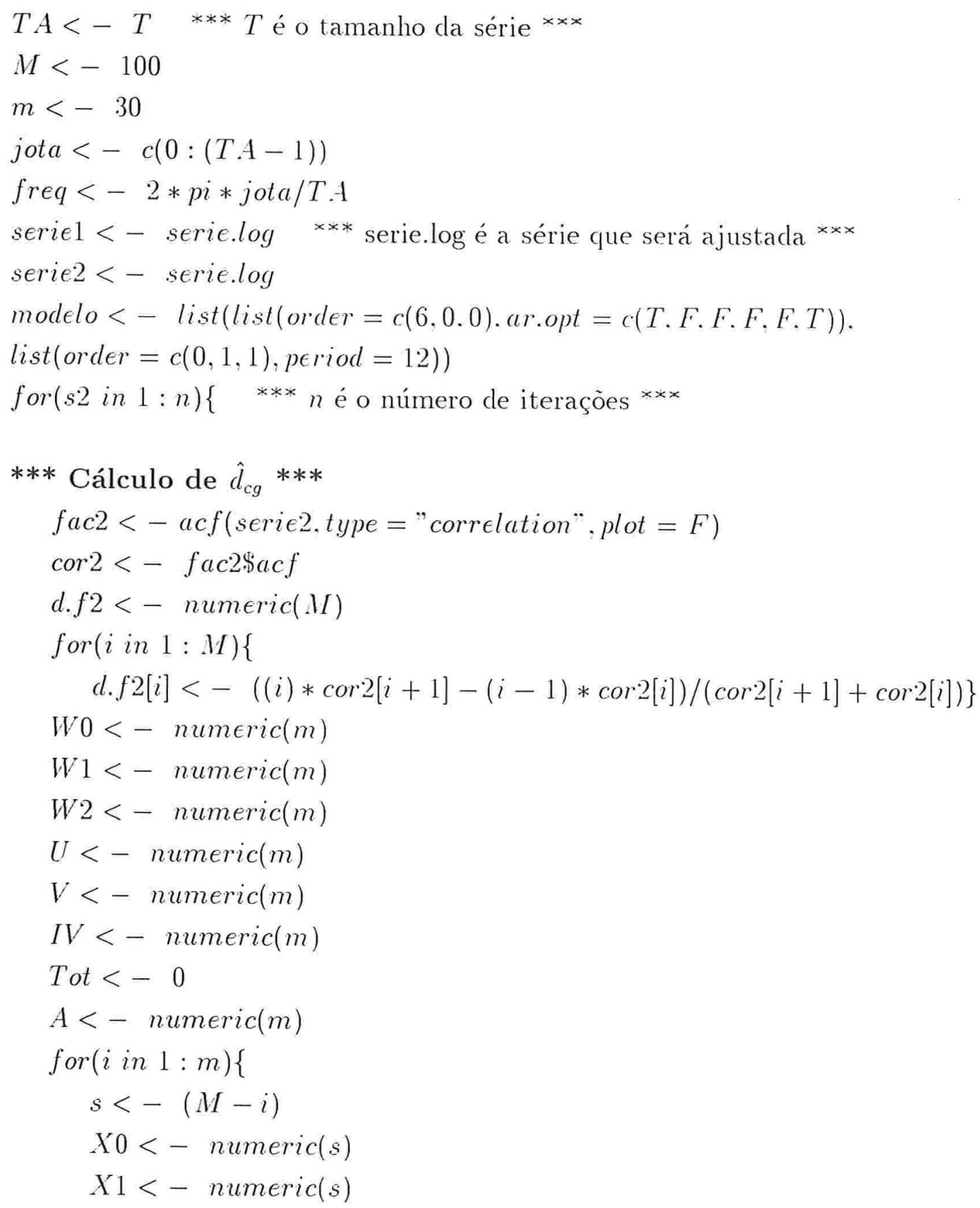




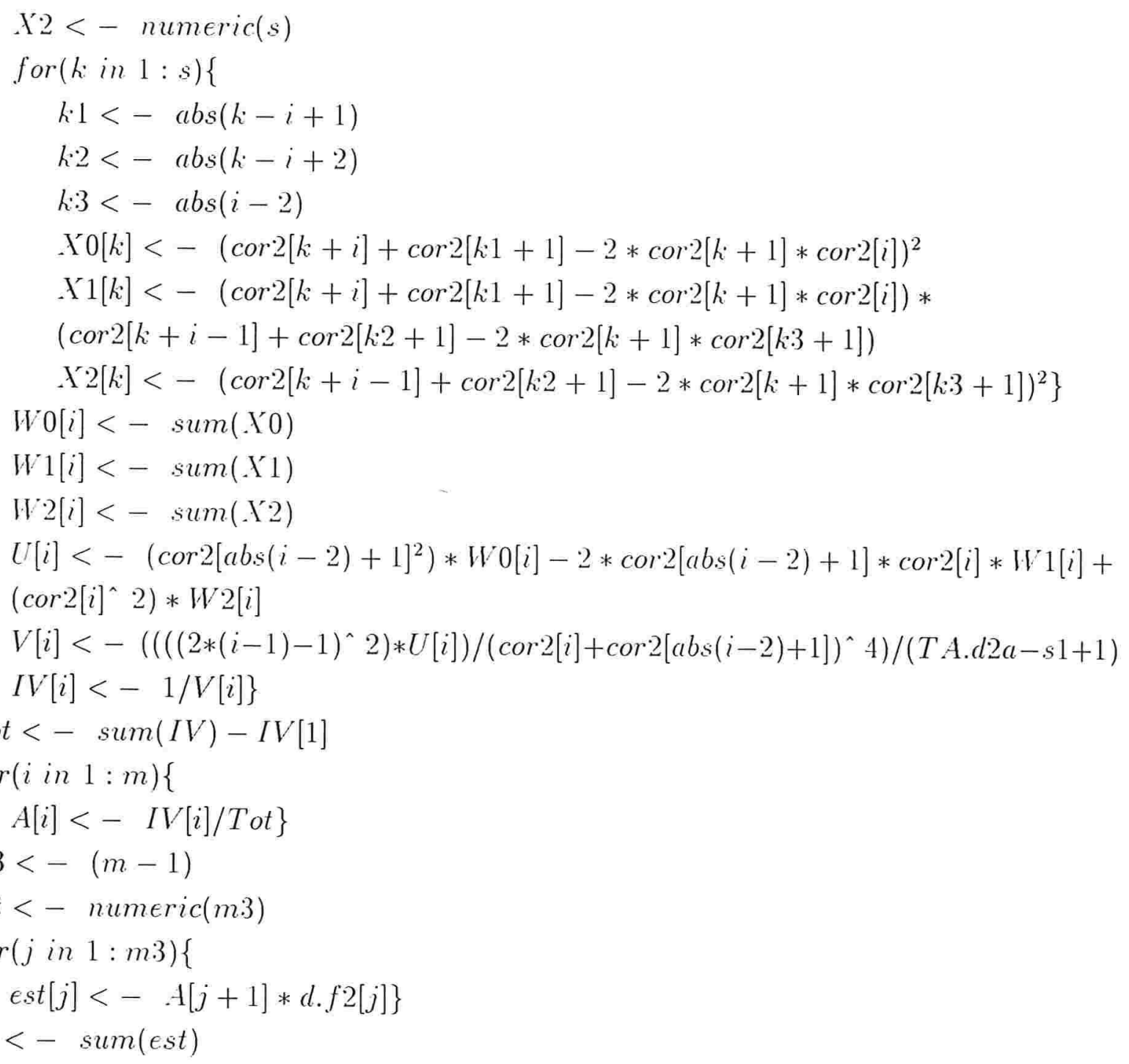

\section{*** Filtro do parâmetro de longa memória ***}

f2.t $<-$ numeric $(T . A)$

for $(j$ in $1: T A)\{$

$f l<-$ numeric $(T . A)$

for $(t 2$ in $1: T A)\{$

$f l[t 2]<-\operatorname{serie} 1[t 2] * \exp (-1 i *$ freq $[j] *(t 2-1))\}$

$f 2 . t[j]<-\operatorname{sum}(f l)\}$

$u 2 . t<-((1-\exp (-1 i * f r e q)) \wedge d 2) * f 2 . t$ 
APENDICE A. PROGRAMAS PARA ESTIMAÇAOO DOS PARÂAIETROS

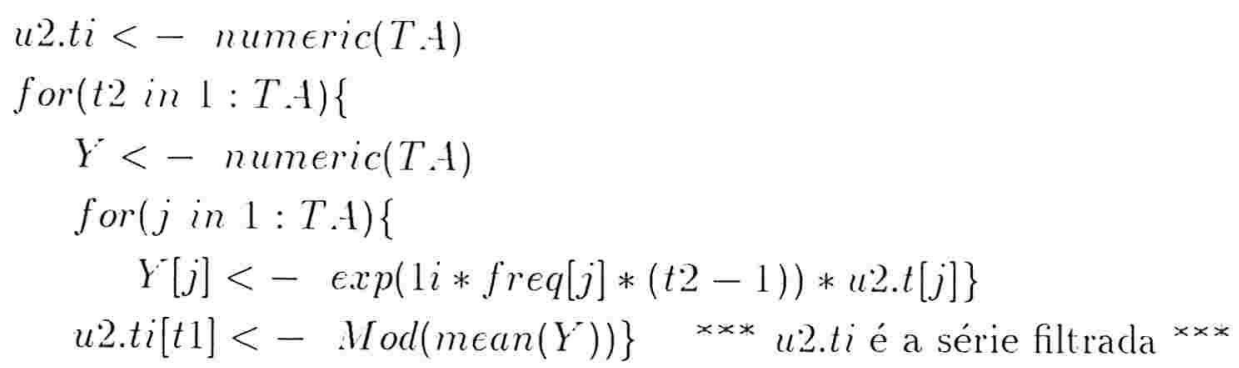

*** Ajuste do modelo $S . A R I M A(6,0,0) \times(0.1,1)_{12}$ à série filtrada, u2.ti. *** fit $2<-$ arima.mle $(u 2 . t i$, model $=$ modelo $)$ filtro $2<-$ arima.filt(serie 1, model $=$ fit 2 smodel) serie $2<-$ filtro2Sfilt [31:T.A] - filtro2spred[31:T.A]\} 
3- Programa para a estimação dos parâmetros do modelo $S A R F I M A(6, d, 0) \times(0.1 .1)_{12}$ quando o estimador de $d$ é $\hat{d}_{p}$

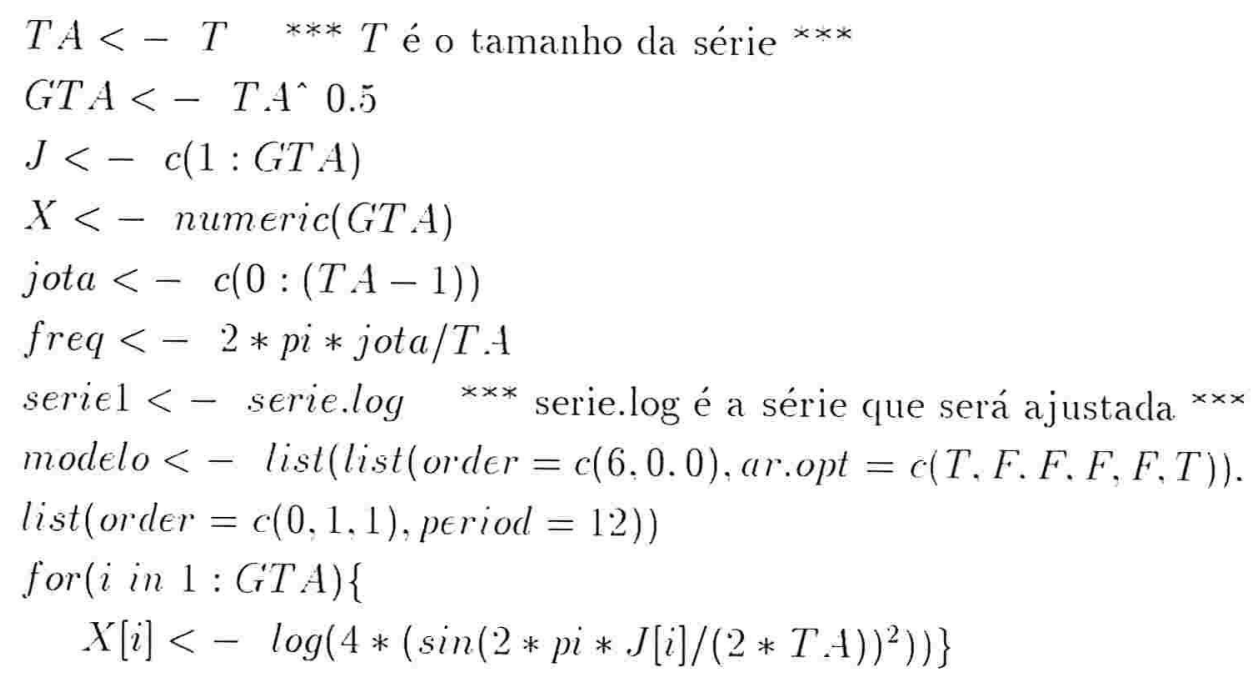


APENDICE A. PROGRAMAS PARA ESTIMAÇÃO DOS P.ARÂMETROS

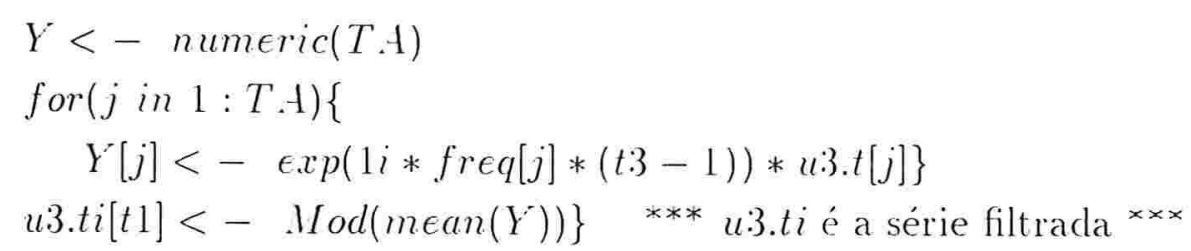

*** Ajuste do modelo $S A R I M A(6,0,0) \times(0,1,1)_{12}$ à série filtrada, u3.ti. *** fit $3<-$ arima.mle $(u 3 . t i$, model $=$ modelo $)$ 
4 - Programa para a estimação dos parâmetros do modelo $S A R F I M A(6, d .0) \times(0,1,1)_{12}$ quando o estimador de $d$ é $\hat{l}_{p s}$

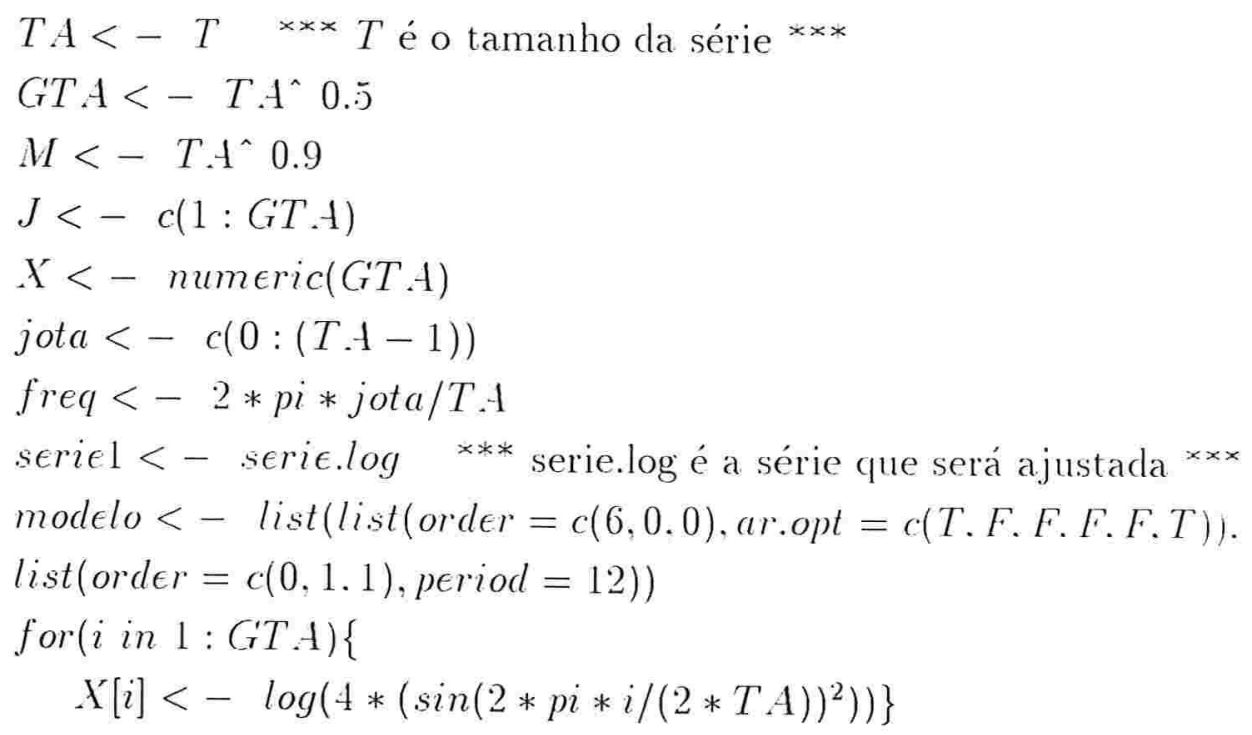




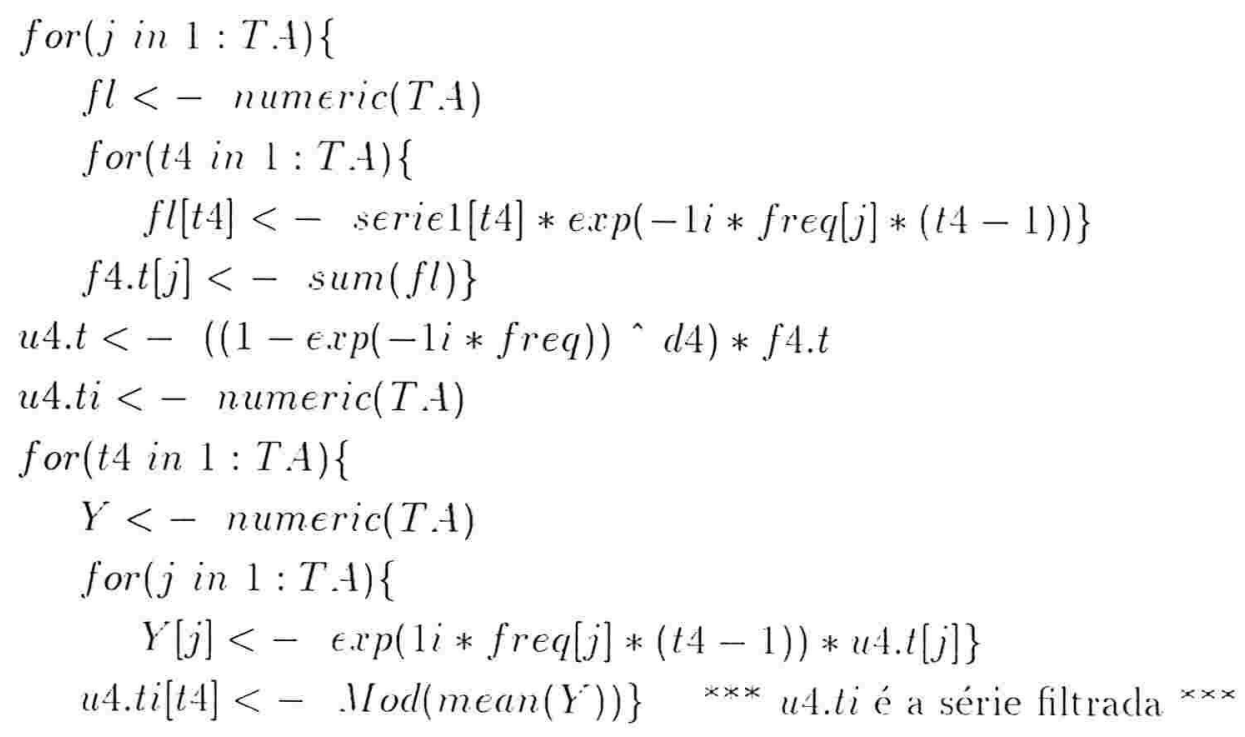

*** Ajuste do modelo $S . A R I M A(6,0,0) \times(0,1.1)_{12}$ à série filtrada, u4.ti. *** fit $4<-$ arima.mle $(u 4 . t i$, model $=$ modelo $)$ 
5 - Programa para a estimação dos parâmetros do modelo $S A R F I . M A(6, d .0) \times(0,1,1)_{12}$ quando o estimador de $d$ é $\hat{d}_{m v}$ Método Iterativo

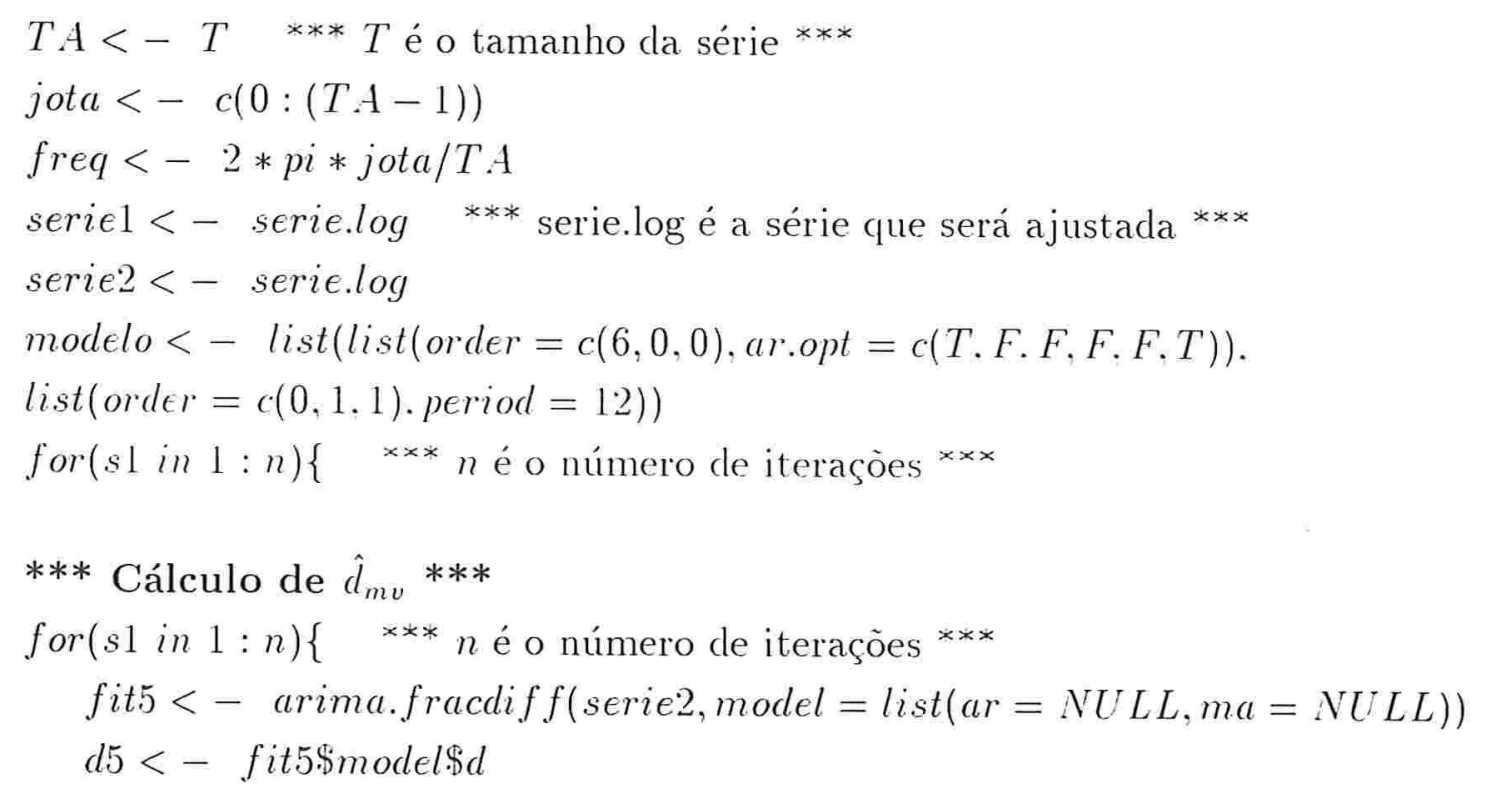

*** Filtro do parâmetro de longa memória $* * *$

f5.t $<-$ numeric $(T . A)$

for $(j$ in $1: T A)\{$

$f l<-\operatorname{numeric}(T A)$

for $(t, 5$ in $1: T A)\{$

$f l[t 5]<-\operatorname{serie} 1[t 5] * \exp (-1 i *$ freq $[j] *(t 5-1))\}$

$f 5 . t[j]<-\operatorname{sum}(f l)\}$

$u .5 . t<-((1-\epsilon x p(-1 i * f r e q)) \wedge$ (i5) *f.5.t

u.5.ti $<-$ numeric(T.A)

for (t.5 in $1: T .4)\{$

$Y<-\operatorname{numeric}(T A)$

for $(j$ in $1: T A)\{$

$Y[j]<-\exp (1 i * \operatorname{freq}[j] *(t 5-1)) * u \bar{J} . t[j]\}$

$u 5 . t i[t 5]<-\operatorname{Mod}(\operatorname{mean}(Y))\} \quad * * * u 5 . t i$ é a série filtrada ${ }^{* * *}$ 
*** Ajuste do modelo $S . A R I M A(6,0,0) \times(0.1,1)_{12}$ à série filtrada, u5.ti. *** fit. $5<-$ arima.mle (u.5.ti, model $=$ modelo $)$ filtro: $<<-$ arima.filt (serie1, model $=$ fit.s.smodel $)$ serie $2<-$ filtro: $\$$ filt $[31:$ T.A] - filtro.5pred $[31: T . A]\}$ 
6 - Programa para a estimação dos parâmetros do modelo $S A R F I . M A(6, d, 0) \times(0.1 .1)_{12}$ quando o estimador de $d$ é $\hat{l}_{w}$ Método Iterativo

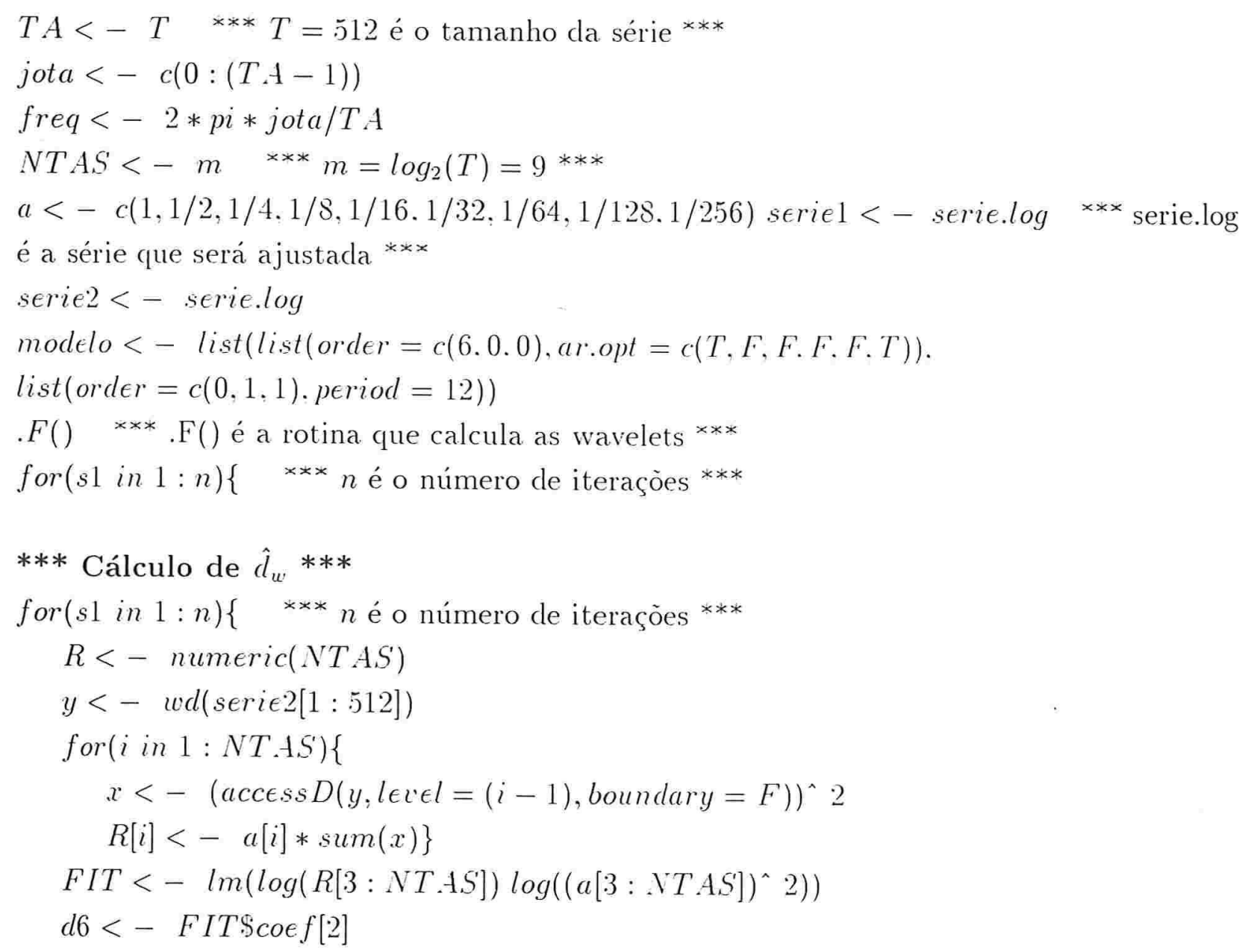

*** Filtro do parâmetro de longa memória ***

f6.t $<-$ numeric $(T . A)$

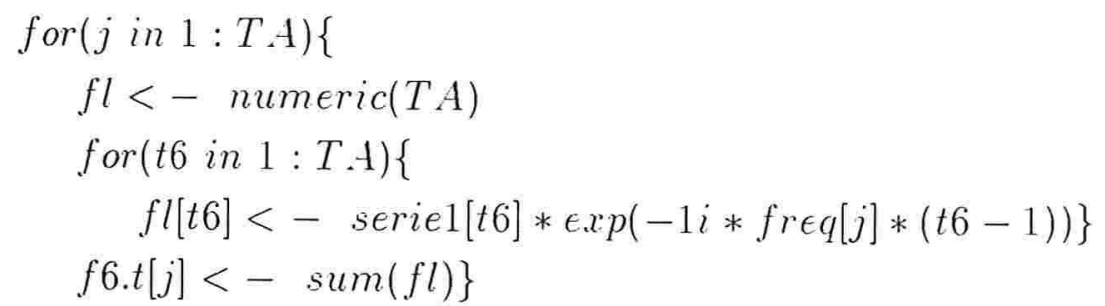




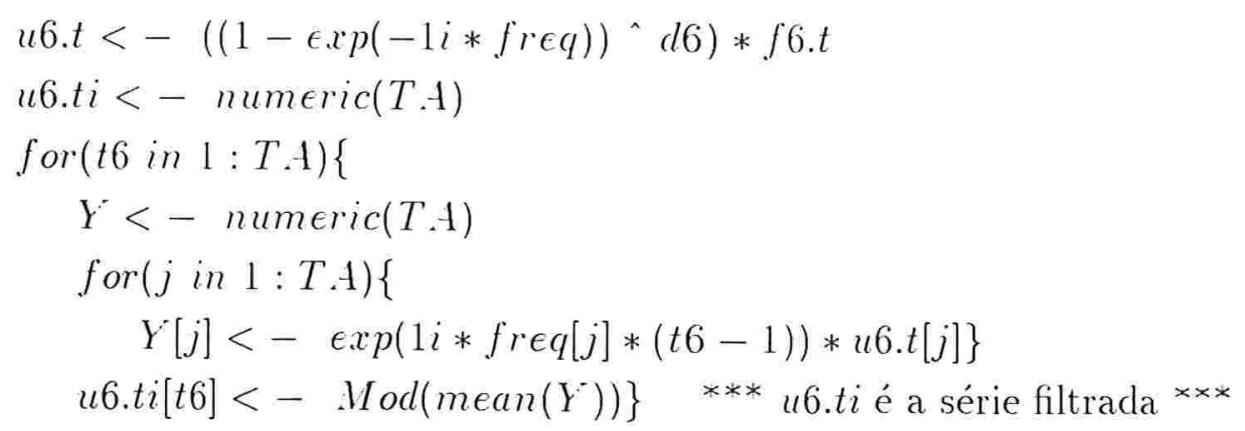

*** Ajuste do modelo $S . A R I . M A(6,0,0) \times(0,1,1)_{12}$ à série filtrada, u6.ti. *** fit6 $<-$ arima.mle $(u 6 . t i$. model $=$ modelo $)$ filtro6 $<-$ arima.filt (serie1. model $=$ fit6smodel $)$ serie $2<-$ filtro6s filt $[31:$ T.A] - filtro6spred $[31:$ T.A]\} 


\section{Apêndice B}

\section{Programa para previsão do modelo $\operatorname{SARFIMA}(6, d, 0) \times(0,1,1)_{12}$}

$d<-$ d.est $\quad * * *$ d.est é o valor estimado do parâmetro $d$.

phi $<-$ philest $\quad * * *$ phil.est é o valor estimado do parâmetro o.

phi6 $<-$ phi6.est $\quad * * *$ phi6.est é o valor estimado do parâmetro $\phi_{6}$.

a $1<-$ numeric $(T 1) \quad{ }^{* * *} T 1<T$, onde $\mathrm{T}$ é o tamanho da série. ${ }^{* * *}$

for $(i$ in $1: T 1)\{$

$a 1[i]<-\quad-\operatorname{gamma}(i-d) /(\operatorname{gamma}(-d) * \operatorname{gamma}(i+1))\}$

$b 1<-$ numeric $(T !)$

$b 1[1]<-a 1[1]+$ phi 1

$b 1[2]<-a 1[2]-$ phi1 $* a 1[1]$

$b 1[3]<-a 1[3]-$ phi $1 * a 1[2]$

$b 1[4]<-a 1[4]-$ phi1 $* a 1[3]$

$b 1[5]<-a 1[5]-$ phi1 $* a 1[4]$

$b 1[6]<-a 1[6]-$ phi1 $* a 1[5]+$ phi6

for $(j$ in $7: T 1)\{$

$b 1[j]<-a 1[j]-$ phi $1 * a 1[j-1]-$ phi6 $* a 1[j-6]\}$

model $1<-\operatorname{list}($ list $(a r=b 1)$, list $($ ma $=$ theta12.est. period $=12)) \quad{ }^{\times \times *}$ theta12.est é o valor estimado do parâmetro $\theta_{12}{ }^{* * *}$

pred $1 m 2<-$ arima.forecast $($ serie, $n=12$, model $=$ model 1$) \quad * * \star$ serie é a série ajustada pelo modelo $S A R F I M A(6, d, 0) \times(0,1,1)_{12} \times * *$ 


\section{Apêndice C}

\section{Logarítmo da série hidrológica}

\section{Rio São Marcos}

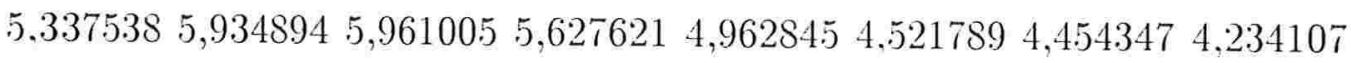
$4,2195084,1896554,2046934,499810 \quad 5,214936 \quad 5,375278 \quad 5,0998664$ 4,682131 $\begin{array}{lllllllll}4,290459 & 4,262680 & 4,043051 & 3,713572 & 3,401197 & 3,931826 & 4,204693 & 4,875197\end{array}$ $5.6489745,4424185,105945 \quad 5,017280 \quad 4,564348$ 4,290459 4,143135 3,806662 $3.713572 \quad 3,9120234,110874 \quad 4,828314 \quad 5,0172804,7706854,762174 \quad 4,521789$ $4.127134 \quad 3,526361 \quad 3,3322053,091042 \quad 3,258097 \quad 3,583519 \quad 3,433987 \quad 3,828641$ $5.3981635,5645205,4071725,4680604,934474$ 4,430817 4,077537 3.931826 3,295837 3,784190 3,951244 4,521789 4,564348 4,110874 5,241747 4,58496 $\begin{array}{llllllllll}4,356709 & 3,891820 & 3,761200 & 3,465736 & 3,295837 & 3,332205 & 3,737670 & 4,17438\end{array}$ $5.0106354,0253524,3944494,543295 \quad 4,2046934,0073333 \quad 3,637586 \quad 3,3322205$ $3.178054 \quad 3,2958374,2766664,9767345,1298994,7361984,753590 \quad 4,219508$ $3.9512443,784190 \quad 3,713572 \quad 3,1780542,484907 \quad 2,708050 \quad 3,583519 \quad 4,795791$ $\begin{array}{lllllllll}5.231109 & 5,267858 & 4,394449 & 4,584967 & 4,043051 & 3,871201 & 3,663562 & 3,401197\end{array}$

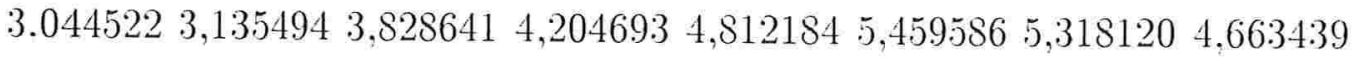
$\begin{array}{llllllll}4.317488 & 4,007333 & 3,688879 & 3,367296 & 3,135494 & 3,295837 & 4,499810 & 4,330733\end{array}$

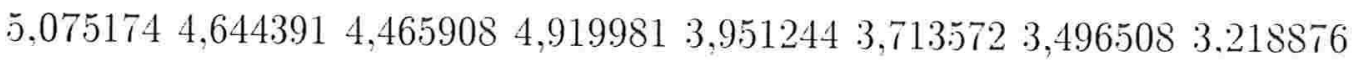
$3.178054 \quad 3,433987 \quad 3,891820 \quad 4,584967 \quad 5,003946 \quad 4,919981 \quad 5,4680604,919981$ 
4,343805 4,804021 4.290459 3.610918 3,496.508 3.433987 4.290459) 4.727.388 $5.7714415 .6767545 .5412644 .6634394 .430817+.317488$ 4.094345 3.688879

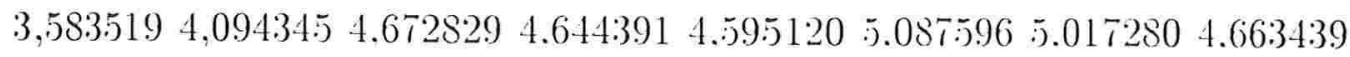
$\begin{array}{llllllllll}4,356709 & 3,931826 & 3.737670 & 3.465736 & 3.258097 & 3.295837 & 4.356709 & 4.4426 .51\end{array}$ $4,8283145,5834965,5012585,1239645,0172804.5432954 .5307334$ 4.0073333 3,737670 4,0253524,605170 4,543295 5,74939:3 5.375278 5,641907 5,468060 $4,7004804,3944494,2904594,0073333 \quad 3.850148$ 3.891820 4,110874 4.691348 $5,0039465,2364425,8318825.081404 \quad 4,7535904.382027 \quad 4,174387 \quad 3.970292$ $3,784190 \quad 3,784190 \quad 3,7376704.8283145 .298317 \quad 5.2149365,5161004.867534$

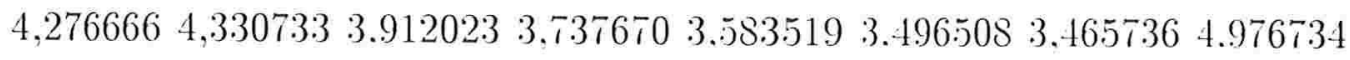
$5,1817845,8435445.4595864,7874924.55387$ 4.276666 4.127134 3.891820 3,610918 4,043051 4.5.5877 4.418841 4.812184 4.912655 5.017280 4.948760 4,406719 4,077537 3,737670 3,25809-2,4849073.5.5348 4.4773337 5.075174 $5,1059454,9628455.0106355,0304384.543295 \quad 4.382027 \quad 4.0430513 .806662$ 3,33220.5 3,433987 3,496508 4,17438 4,584967 5,472271 5,513429 5.030438 4,844187 4,595120 4,369448 4,07ז537 3,891820 3,713572 4,543295 5,117994 $4,6634394,6151215.2781155,1984974,7874924,465.908$ 4,1431355 3,713572 $3,6375864,418841 \quad 4,7621745,1873864,912655$ 5.303305 4,77.91223 4,499810 4,110874 3,761200 3,258097 2,772589 2,397895 1.791759 4,234107 4,812184 $4,976734 \quad 4,9836074,6728294.8598124 .33073334,025352 \quad 3,5555348$ 2,995732 2,302585 3,891820 4,532599 4.976734 4,98:3607 4.574711 5,147494 4,682131 $\begin{array}{llllllllll}4,844187 & 4,584967 & 4.276666 & 3.688879 & 3.761200 & 3.526361 & 4.736198 & 5.513128\end{array}$ $5,459586 \quad 5,6021195,5134295,5,3518.585,0106354.8040214,6634394.418841$ $4,2046934,1743874,5217894,98360$-4,905275 5.135798 5,062595 5,023881 $\begin{array}{llllllll}4,615121 & 4,510860 & 4,394449 & 3.988984 & 4,0253352 & 4.060443 & 3,761200 & 4,234107\end{array}$ $5,318120 \quad 4,962845$ 5.164786 4,912655 4,532599 $4.276666 \quad 3,988984$ 3,5,5.5348

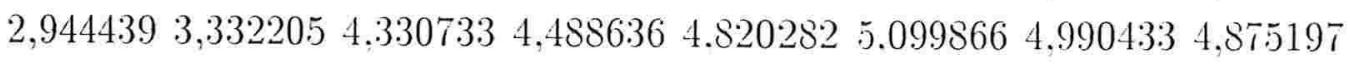
$\begin{array}{lllllllll}4,584967 & 4,290459 & 4.0253552 & 3.610918 & 2.708050 & 3.218876 & 4,043051 & 4.356709\end{array}$ 4,\$67534 5,056246 4,812184 4,779123 4,615121 4,418841 4,234107 4,094345 3,583519 2,772.589 3.970292 4,510860 4,727388 5,1.53292 4,820282 4,718499 4,418841 4,1431355 3.828641 3,583519 $3.218876 \quad 3,713572 \quad 4,248495 \quad 4,709530$

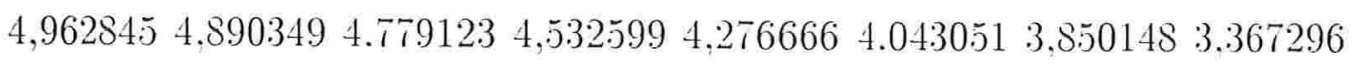
2,639057 2,564949 3.951244 3,761200 5.j56828 5.789960 4,962845 4, т.53.590 


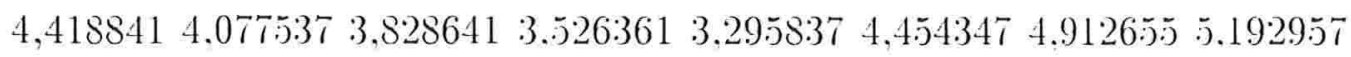
$5,6664275,924256 \quad 6.2205905 .4510385 .0172804 .6539604 .465908$ 4.219.508 3,828641 4,369448 4,9126.5.5 5.541264 6,045005 6,424869 5,68:3580 5.3.518.58 $5,1298994,8283144,6051704.369448 \quad 4,1431354,4.54347$ 4,812184 5.634790 $5,6489745,9295896,2324485,4467375,3181204,9698134,454347$ 4.343805 $4,174387 \quad 3,9318264,8362825,6454475,7037826.0014155,953243$ 5.379897 $4,9972124,7361984,4773374,2904594,1271344,369448$ 4,418\$41 5.356.586 $5,0106355,4424185,2678.58$ 4,770685 4,521789 4,143135 3.912023 3.637.586 3,401197 3,806662 5,247024 5,313206 6,129050 5,918894 5,556828 5.003946 $\begin{array}{lllllllll}4,727388 & 4,234107 & 4,007333 & 3,828641 & 3,637586 & 4,488636 & 4.682131 & 4.488636\end{array}$ $\begin{array}{lllllllll}4,077537 & 4,043051 & 4,262680 & 4,276666 & 3.912023 & 3.737670 & 3.367296 & 3.218876\end{array}$ 3,258097 4,110874 4,905275 5,697093 5,075174 5.0625.95 5.147494 5.123964 4,6634:39 4,369448 4,174387 3,850148 $3,637586 \quad 4,219508$ 5,111988 5.356586 $5,3327195,393628 \quad 5,34233345,5214614,8598124,553877 \quad 4,369448$ 4.0775.37 3,806662 4,477337 5,293305 5,283204 5,236442 4,9199815,921578 5.752573 $5,2311094,8751974,624973 \quad 4,418841 \quad 4,094345 \quad 4,369448 \quad 4,2904594.663439$ $5,2203565,347108$ 4,736198 4,95.5827 4,615121 4,290459 4,110874 3.i61200 $3,4011973,7135724,5747114,736198$ 